\title{
Semi-Analytic Reconstruction of Flux in Finite Volume Formulations
}

\author{
Peter A Gnoffo \\ NASA Langley Research Center \\ Hampton VA 23681
}

\begin{abstract}
Semi-analytic reconstruction uses the analytic solution to a second-order, steady, ordinary differential equation (ODE) to simultaneously evaluate the convective and diffusive flux at all interfaces of a finite volume formulation. The second-order ODE is itself a linearized approximation to the governing first- and second- order partial differential equation conservation laws. Thus, semi-analytic reconstruction defines a family of formulations for finite volume interface fluxes using analytic solutions to approximating equations. Limiters are not applied in a conventional sense; rather, diffusivity is adjusted in the vicinity of changes in sign of eigenvalues in order to achieve a sufficiently small cell Reynolds number in the analytic formulation across critical points. Several approaches for application of semi-analytic reconstruction for the solution of one-dimensional scalar equations are introduced. Results are compared with exact analytic solutions to Burger's Equation as well as a conventional, upwind discretization using Roe's method. One approach, the end-point wave speed (EPWS) approximation, is further developed for more complex applications. One-dimensional vector equations are tested on a quasi one-dimensional nozzle application. The EPWS algorithm has a more compact difference stencil than Roe's algorithm but reconstruction time is approximately a factor of four larger than for Roe. Though both are second-order accurate schemes, Roe's method approaches a grid converged solution with fewer grid points. Reconstruction of flux in the context of multi-dimensional, vector conservation laws including effects of thermochemical nonequilibrium in the Navier-Stokes equations is developed.
\end{abstract}

\section{Nomenclature}

Bold face, lowercase variable names refer to vectors. Bold face, uppercase variable names refer to matrices.

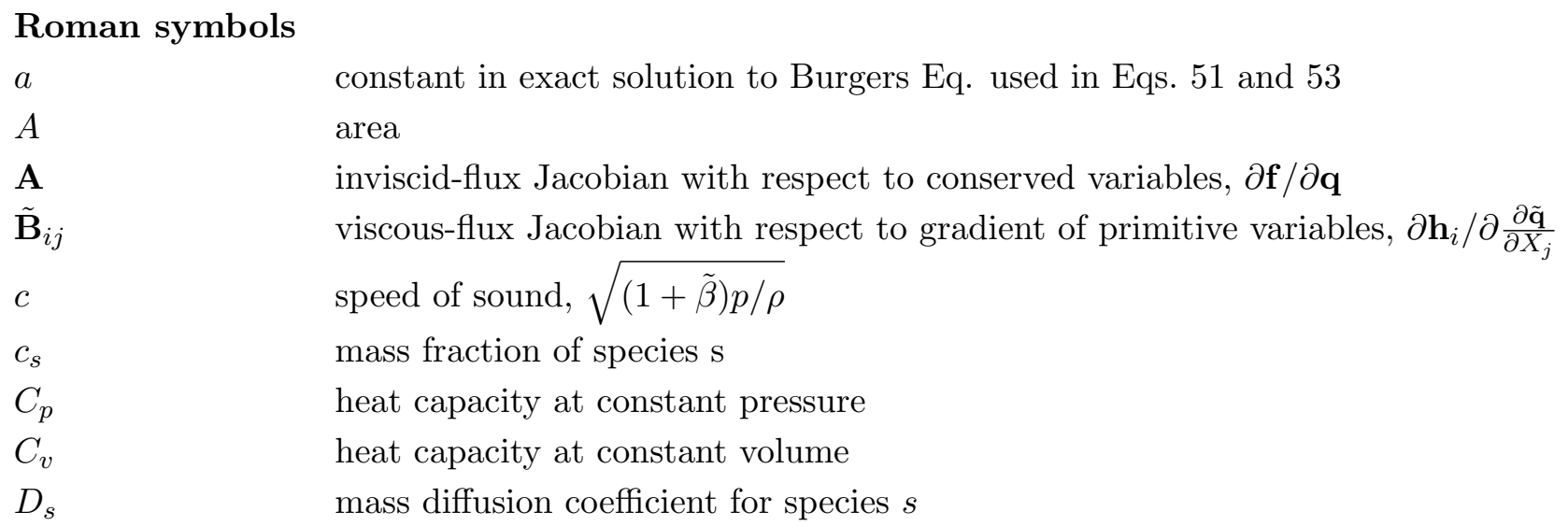




\begin{tabular}{|c|c|}
\hline Roman symbols, & continued \\
\hline$e$ & energy per unit mass \\
\hline$E$ & total energy per unit mass, $e+\tilde{\alpha}$ \\
\hline $\mathbf{f}, f$ & inviscid flux (vector, scalar) \\
\hline $\mathbf{h}, h$ & viscous flux (vector, scalar) \\
\hline$h_{s, t r}$ & translational + rotational enthalpy for species $s$ \\
\hline$h_{s, v}$ & vibrational + electronic enthalpy for species $s$ \\
\hline$H$ & total enthalpy, $E+p / \rho$ \\
\hline$k$ & thermal conductivity \\
\hline$L_{2}$ & error norm, sum of squares \\
\hline$l_{x}, l_{y}, l_{z}$ & components of unit vector in $Y$ direction \\
\hline$m_{x}, m_{y}, m_{z}$ & components of unit vector in $Z$ direction \\
\hline$M$ & Mach number \\
\hline$\tilde{M}$ & mixture molecular weight \\
\hline$\tilde{M}_{s}$ & molecular weight of species $s$ \\
\hline$n_{x}, n_{y}, n_{z}$ & components of unit vector in $X$ direction \\
\hline$p$ & pressure \\
\hline $\operatorname{Pr}$ & Prandtl number, $\mu C_{p} / \tilde{\eta}$ \\
\hline $\operatorname{Pr}_{t r}$ & Prandtl number for translational + rotational energy, $\mu C_{p} / \tilde{\eta}_{t r}$ \\
\hline $\operatorname{Pr}_{v}$ & Prandtl number for vibrational + electronic energy, $\mu C_{p} / \tilde{\eta}_{v}$ \\
\hline $\mathbf{q}, q$ & conserved variable (vector, scalar) \\
\hline $\mathbf{q}^{\prime}, q^{\prime}$ & characteristic variable (vector, scalar) \\
\hline$\tilde{\mathbf{q}}$ & primitive variable vector \\
\hline$r_{f M 0}, r_{f M 1}$ & reconstruction factors, Eq. 40 \\
\hline $\mathbf{R}$ & matrix of right eigenvectors of $\mathbf{A}$ \\
\hline $\bar{R} e$ & reference cell Reynolds number for edge, Eq. 45 \\
\hline$R e_{L}$ & cell Reynolds number referenced to node L, Eq. 39 \\
\hline$R e_{R}$ & cell Reynolds number referenced to node R, Eq. 39 \\
\hline $\operatorname{Rex}$ & cell Reynolds number, $\frac{\lambda\left(x-x_{0}\right)}{\nu}$ \\
\hline $\mathbf{s}, s$ & source term (vector, scalar) \\
\hline $\mathrm{Sc}_{s}$ & Schmidt number for species $s, \mu \rho D_{s}$ \\
\hline$t$ & time \\
\hline$T$ & temperature \\
\hline $\mathbf{T}$ & transform from primitive to conservative variables, $\mathbf{T} d \tilde{\mathbf{q}}=d \mathbf{q}$ \\
\hline$u, v, w$ & Cartesian velocity components \\
\hline$U, V, W$ & velocity components in rotated system \\
\hline$x, y, z$ & reference Cartesian coordinates \\
\hline$X, Y, Z$ & rotated Cartesian system \\
\hline
\end{tabular}




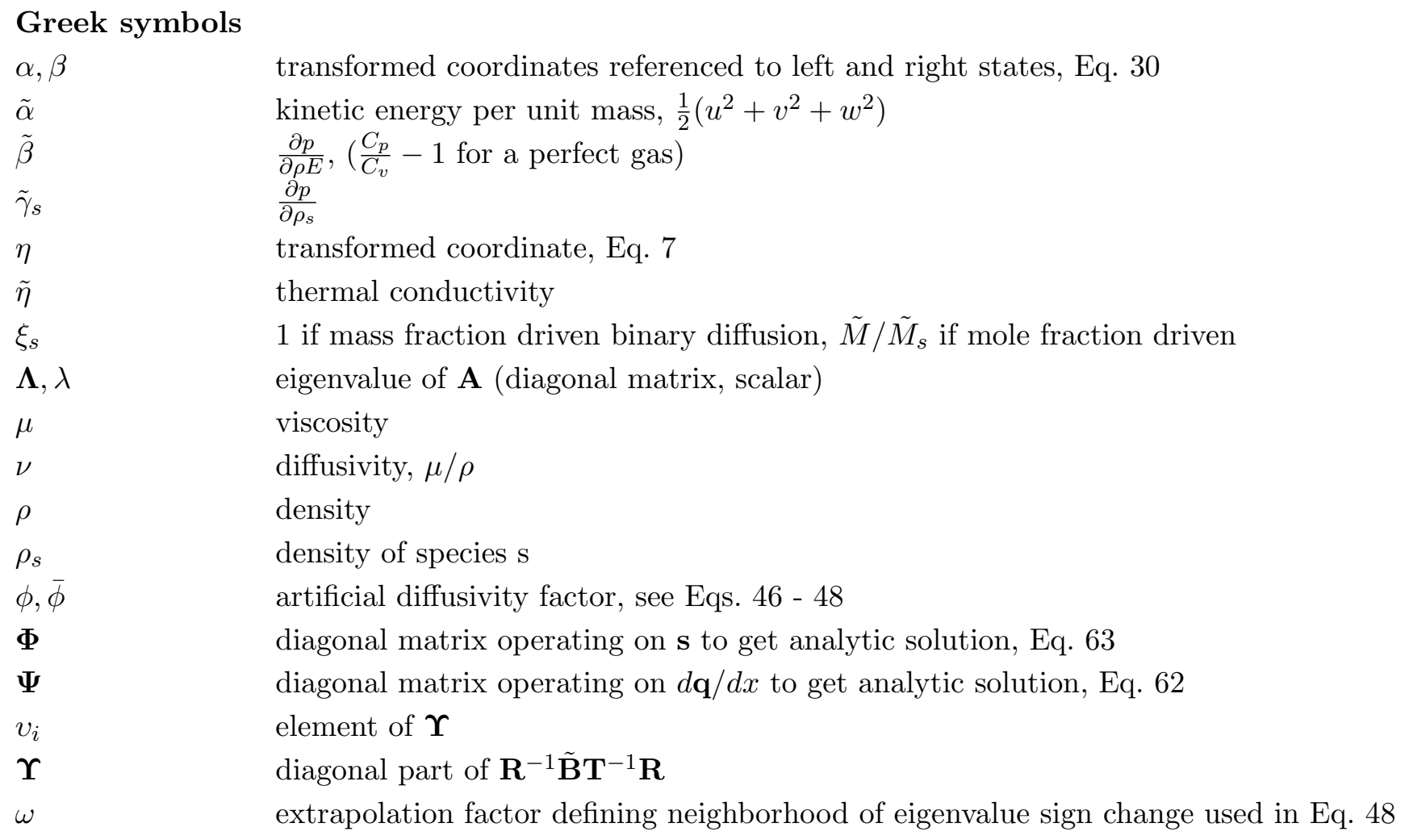

\section{Subscripts}

$1,2,3$

$A, B, C, D$

$C W S$

e

$i, j$

$L$

$M$

$R$

Roe

tr

$v$ referring to $X, Y, Z$ directions, respectively

nodes of tetrahedron

constant wave speed formulation

exact

node index or vector element

left node

dummy index for left or right node

right node

Roe formulation

translational + rotational energy component

vibrational + electronic energy component

\section{Introduction}

The evolution of structured grid to unstructured-grid techniques for flow simulation is motivated by the relative ease of generating an initial grid over a complex configuration and the relative flexibility in adapting to flow features. ${ }^{1}$ Feature adaptation is particularly important in hypersonic flow simulation. The interaction of shocks, boundary layers, free shear-layers, and vortices spawn ever more complex flow structures at oblique angles to flow boundaries and upstream feature orientation. Such interactions are encountered in scram-jet engines, across compression surfaces, protuberances, and deflected control surfaces, around reaction control system jets, and even in the wake of simple blunt bodies. Inadequate resolution of these structures in a flow simulation adversely impacts the prediction of aerodynamic forces and surface heating. 
However, before unstructured simulations can be routinely applied to hypersonic flow simulations, a serious deficiency in simulation quality must be corrected. Unstructured grid simulations of hypersonic flows poorly predict stagnation region heating using high aspect ratio tetrahedra across the boundary layer. The deficiency is readily apparent in a simple test problem of hypersonic flow over a cylinder in which a biased, unstructured grid is used that includes spanwise resolution. ${ }^{2}$ In essence, the grid admits 3D flow for a case in which 2D flow is expected. Some jags in the shock capture process induce irregular entropy distribution in streamlines heading toward the stagnation point. On top of this non-uniformity, the element diagonals obliquely cut across the boundary layer inducing cross flow. The simulated flow quality is highly dependent on the flow limiters applied within the algorithm but, so far, there has been no algorithm fix identified which cures the problem in the context of the underlying, quasi one-dimensional reconstruction technique. The problem can be masked through use of prisms across the boundary layer with faces parallel or orthogonal to the gradient vector. However, unless one is prepared to abandon tetrahedra and automatically introduce and align prisms in all free shear layers and wakes then one must seek an algorithmic solution.

The one-dimensional nature of reconstruction in current finite-volume methods is suspected to be a major contributor to the problem cited above. Reconstruction of flux across a cell face is based on a Riemann problem for a $1 \mathrm{D}$ discontinuity cross the cell face. ${ }^{3-6}$ The reconstruction process does not consider the principle flow direction - it is completely a function of the local grid orientation. In this sense, a structured grid has advantages over an unstructured grid crossing a single shock or boundary layer. All surfaces of the structured grid can be made parallel or orthogonal to the principle flow direction and gradient vectors. A tetrahedron will always have at least one edge oblique to the flow direction and gradient vector but is constrained to use this direction in the flux reconstruction process.

This inherent difficulty with tetrahedra was a primary motivation to develop an algorithm for multidimensional flux reconstruction. The semi-analytic reconstruction introduced here uses a solution to the linearized conservation equations to define the variation of dependent variables between cells. Application of a semi-analytic approach is also inspired by success achieved with a post-processing algorithm that couples analytic solutions to CFD using an integral boundary layer approach for analyzing global changes to surface catalysis on heating ${ }^{7}$ and on a triple deck approach $^{8,9}$ for analyzing local changes to surface boundary conditions on heating. The challenge to the semi-analytic solution approach is the assumption of a continuous solution, even in the presence of shocks, that implicitly requires physical dissipation be included in the derivation. Effects of source terms on the reconstructed flux are also included in the derivation; an effect usually ignored in finite-volume reconstruction although it has been discussed previously by Van Leer. ${ }^{10}$

Semi-analytic reconstruction developed herein uses the analytic solution to a second-order, steady, ordinary differential equation (ODE) to simultaneously evaluate the convective and diffusive flux at all interfaces of a finite volume formulation. The second-order ODE is itself a linearized approximation to the governing first- and second- order partial differential equation (PDE) conservation laws. Thus, semi-analytic reconstruction defines a family of formulations for finite volume interface fluxes using analytic solutions to approximating equations. Limiters are not applied in a conventional sense; rather, diffusivity is adjusted in the vicinity of changes in sign of eigenvalues in order to achieve a sufficiently small cell Reynolds number in the analytic formulation across critical points. Several approaches for application of semi-analytic reconstruction for the solution of one-dimensional scalar equations are introduced in Sec. III. Results are compared with exact analytic solutions to Burger's Equation as well as a conventional, upwind discretizations using Roe's scheme. One approach, the end-point wave speed (EPWS) approximation, is further developed in Sec. IV for more complex applications. One-dimensional vector equations are tested on a quasi one-dimensional nozzle application. These test problems include strong shocks and source 
terms. Possible approaches to higher-order accurate approximations are suggested in the Appendix, though these ideas have not been tested on anything more than a 1D scalar equation.

The ultimate proof of the algorithm in $3 \mathrm{D}$ will be the cylinder problem discussed above. These tests have not been completed but reconstruction of flux in the context of multi-dimensional, vector conservation laws including effects of thermochemical nonequilibrium in the Navier-Stokes equations is developed in Sec. V for future testing.

\section{One-Dimensional Scalar Equation}

A one-dimensional, scalar conservation law including both convection and diffusion can be expressed by the following equation.

$$
\frac{\partial q}{\partial t}+\frac{\partial}{\partial x}(f-h)=s
$$

A numerical solution to Eq. 1 written in conservative form must guarantee that the convective $(f)$ and diffusive $(h)$ flux exiting cell $i$ is exactly balanced by that entering cell $i+1$. Such a balance is enforced in the following difference equation.

$$
\frac{q^{n+1}-q^{n}}{\Delta t}+\frac{\left[(f-h)_{i+1 / 2}-(f-h)_{i-1 / 2}\right]}{\left(x_{i+1 / 2}-x_{i-1 / 2}\right)}=s_{i}
$$

Eq. 2 requires a reconstruction of $f$ and $h$ on cell boundaries. In a typical formulation the convective term and diffusive terms are reconstructed independently. For example, in Roe's method, the convective term is defined as follows.

$$
f_{i+1 / 2}=\frac{1}{2}\left[f_{i+1}+f_{i}-\left|\lambda_{i+1 / 2}\right|\left(q_{i+1}-q_{i}+d q_{i+1 / 2}^{l i m}\right)\right]
$$

The variable $\lambda_{i+1 / 2}$ is an approximation to $(d f / d q)_{i+1 / 2}$ that satisfies the relation $\lambda_{i+1 / 2}\left(q_{i+1}-q_{i}\right)=f_{i+1}-f_{i}$. The term $d q_{i+1 / 2}^{l i m}$ provides a second-order formulation of $f_{i+1 / 2}$; various limiter functions may be applied to serve this function. The diffusion term in Eq. 2 is typically defined as follows.

$$
h_{i+1 / 2}=\frac{\left(\nu_{i+1}+\nu_{i}\right)}{2} \frac{\left(q_{i+1}-q_{i}\right)}{\left(x_{i+1}-x_{i}\right)}
$$

Note that the explicit formulation of the convective flux (Eq. 3) and diffusive flux (Eq. 4) are independent of each other and of the source term $s$. The convective flux formulation exploits the continuity of the inviscid flux $f$ even across shocks and slip surfaces where the dependent variable $q$ may not be continuous.

A unified algorithm for simultaneous reconstruction of the convective and diffusive flux, including the influence of a source term, is derived from a semi-analytic solution to the steady state form of Eq. 1. In this approach, the continuity of $q$ is required in the formulation, even across shocks and slip surfaces which acquire finite thickness with the inclusion of physical dissipation. Semi-analytic reconstruction is based on a solution to Eq. 5

$$
\lambda \frac{d q}{d x}=\frac{d}{d x}\left(\nu \frac{d q}{d x}\right)+s
$$

where $\lambda=\frac{d f}{d q}$. 


\section{A. Constant Wave Speed Approximation (CWS)}

\section{Nominal Algorithm}

Approximating the source term $s$, diffusivity $\nu$, and wave speed $\lambda$ in Eq. 5 as constants across the interval $\left[x_{L}, x_{R}\right]$ and introducing, for notational convenience, $x_{L}=x_{i}, x_{0}=x_{i+1 / 2}$, and $x_{R}=x_{i+1}$ yields the following equation.

$$
\lambda_{0} \frac{d q}{d x}=\nu_{0} \frac{d^{2} q}{d x^{2}}+s_{0}
$$

The analytic solution to Eq. 6 with constant coefficients can be derived by inspection or through reference to existing textbooks. However, a coordinate transformation is introduced here for two reasons. First, a non-constant coefficient case is more easily treated in Appendix A. Second, the role of integration constants $q_{0}$ and $(d q / d x)_{0}$ are more clearly defined as are their limiting forms as wave speed goes to zero. Consequently, the analytic solution to Eq. 6 is derived by introducing the following map from $x$ to $\eta$.

$$
\begin{array}{rlc}
\eta(x) & = & \int_{0}^{x-x_{0}} \exp \left[\frac{\lambda_{0}\left(x^{\prime}\right)}{\nu_{0}}\right] d x^{\prime} \\
& = & \frac{\nu_{0}}{\lambda_{0}}\left(\exp \left[\frac{\lambda_{0}\left(x-x_{0}\right)}{\nu_{0}}\right]-1\right) \\
\frac{d \eta}{d x} & = & \exp \left[\frac{\lambda_{0}\left(x-x_{0}\right)}{\nu_{0}}\right] \\
\frac{d^{2} \eta}{d x^{2}} & = & \frac{\lambda_{0}}{\nu_{0}} \frac{d \eta}{d x}
\end{array}
$$

In the limit of $\lambda_{0} \rightarrow 0$ the mapping defined by Eq. 7 becomes

$$
\begin{array}{rlc}
\eta & = & x-x_{0} \\
\frac{d \eta}{d x} & = & 1 \\
\frac{d^{2} \eta}{d x^{2}} & = & 0
\end{array}
$$

Substitution of Eq. 7 (or Eq. 8 if $\lambda_{0}=0$ ) into Eq. 6 yields the following.

$$
\frac{d^{2} q}{d \eta^{2}}=-\frac{s_{0}}{\nu_{0}}\left(\frac{d x}{d \eta}\right)^{2} .
$$

The general solution to Eq. 9 is

$$
\begin{aligned}
q_{\text {general }} & =q_{0}+\left(\frac{d q}{d \eta}\right)_{0} \eta \\
& =q_{0}+\left(\frac{d q}{d x}\right)_{0}\left(\frac{d x}{d \eta}\right)_{0} \eta \\
& =q_{0}+\left(\frac{d q}{d x}\right)_{0} \frac{\nu_{0}}{\lambda_{0}}\left(\exp \left[\frac{\lambda_{0}\left(x-x_{0}\right)}{\nu_{0}}\right]-1\right)
\end{aligned}
$$

The particular solution to Eq. 9 is derived as follows. First, integrate both sides of Eq. 9, returning to independent variable $x$ on the left hand side to simplify the integration.

$$
\begin{aligned}
\left(\frac{d q}{d \eta}\right)_{\text {particular }} & =-\frac{s_{0}}{\nu_{0}} \int_{0}^{\eta}\left(\frac{d x}{d \eta}\right)^{2} d \eta^{\prime} \\
& =-\frac{s_{0}}{\nu_{0}} \int_{0}^{x-x_{0}} \exp \left[-\frac{2 \lambda_{0}\left(x^{\prime}\right)}{\nu_{0}}\right] \frac{d \eta}{d x} d x^{\prime} \\
& =\frac{s_{0}}{\lambda_{0}}\left(\exp \left[-\frac{\lambda_{0}\left(x-x_{0}\right)}{\nu_{0}}\right]-1\right)
\end{aligned}
$$

Note that $\left(\frac{d q}{d \eta}\right)_{\text {particular }, 0}=0$; consequently, the gradient $\left(\frac{d q}{d x}\right)_{0}$ is fully defined by the general solution for this case where $s, \lambda$, and $\nu$ are treated as a constants over the interval. Integrate both 
sides of Eq. 11 in the same manner to complete the derivation of the particular solution.

$$
\begin{aligned}
q_{\text {particular }} & =\int_{0}^{\eta}\left(\frac{d q}{d \eta}\right)_{\text {particular }} d \eta^{\prime} \\
& =\frac{s_{0}}{\lambda_{0}} \int_{0}^{x-x_{0}}\left(\exp \left[-\frac{\lambda_{0}\left(x^{\prime}\right)}{\nu_{0}}\right]-1\right) \frac{d \eta}{d x} d x^{\prime} \\
& =\frac{s_{0}}{\lambda_{0}} \int_{0}^{x-x_{0}}\left(1-\exp \left[\frac{\lambda_{0}\left(x^{\prime}\right)}{\nu_{0}}\right]\right) d x^{\prime} \\
& =\frac{s_{0}}{\lambda_{0}}\left[\left(x-x_{0}\right)-\frac{\nu_{0}}{\lambda_{0}}\left(\exp \left[\frac{\lambda_{0}\left(x-x_{0}\right)}{\nu_{0}}\right]-1\right)\right] \\
& =\frac{s_{0}}{\lambda_{0}}\left[\left(x-x_{0}\right)-\eta(x)\right]
\end{aligned}
$$

In the limit as $\lambda_{0} \rightarrow 0$ the particular solution in Eq. 12 goes to the expected result

$$
q_{\text {particular }}=-\frac{s_{0}}{2 \nu_{0}}\left(x-x_{0}\right)^{2} .
$$

The solution to Eq. 6 in the interval $\left[x_{L}, x_{R}\right]$ may now be expressed.

$$
\begin{aligned}
q\left(x-x_{0}\right) & =q_{\text {general }}+q_{\text {particular }} \\
& =q_{0}+\left(\frac{d q}{d x}\right)_{0} \eta(x)+\frac{s_{0}}{\lambda_{0}}\left[\left(x-x_{0}\right)-\eta(x)\right]
\end{aligned}
$$

The values of $q_{0}$ and $\left(\frac{d q}{d x}\right)_{0}$ needed for reconstruction can be solved implicitly by evaluating Eq. 14 at the endpoints of the interval $\left[x_{L}, x_{R}\right]$ using known values $q_{L}$ and $q_{R}$.

$$
\begin{array}{r}
q_{L}=q_{0}+\left(\frac{d q}{d x}\right)_{0} \eta_{L}+\frac{s_{0}}{\lambda_{0}}\left[\left(x_{L}-x_{0}\right)-\eta_{L}\right] \\
q_{R}=q_{0}+\left(\frac{d q}{d x}\right)_{0} \eta_{R}+\frac{s_{0}}{\lambda_{0}}\left[\left(x_{R}-x_{0}\right)-\eta_{R}\right] \\
q_{0}=\frac{\eta_{R}\left(q_{L}-\frac{s_{0}}{\lambda_{0}}\left(x_{L}-x_{0}\right)\right)-\eta_{L}\left(q_{R}-\frac{s_{0}}{\lambda_{0}}\left(x_{R}-x_{0}\right)\right)}{\eta_{R}-\eta_{L}} \\
\left(\frac{d q}{d x}\right)_{0}=\frac{\left(q_{L}-\frac{s_{0}}{\lambda_{0}}\left(x_{L}-\eta_{L}\right)\right)-\left(q_{R}-\frac{s_{0}}{\lambda_{0}}\left(x_{R}-\eta_{R}\right)\right)}{\eta_{L}-\eta_{R}}
\end{array}
$$

Note that Eqs. 17 and 18 for $q_{0}$ and $(d q / d x)_{0}$ respectively are derived from Eqs. 15 and 16. Equation 17 is an implicit relation in that $\eta_{L}$ and $\eta_{R}$ (and possibly $s_{0}$ ) are functions of $q_{0}$ through $\lambda_{0}$ and $\nu_{0}$. A Newton iteration to solve Eq. 17 for $q_{0}$ is constructed as follows.

$$
\begin{aligned}
& \hat{q}=q_{0}-\frac{\eta_{R}\left(q_{L}-\frac{s_{0}}{\lambda_{0}}\left(x_{L}-x_{0}\right)\right)-\eta_{L}\left(q_{R}-\frac{s_{0}}{\lambda_{0}}\left(x_{R}-x_{0}\right)\right)}{\eta_{R}-\eta_{L}} \\
& \frac{d \hat{q}}{d q_{0}}=1-\frac{d}{d \lambda_{0}}\left(\frac{\eta_{R}}{\eta_{R}+\eta_{L}}\right) \frac{d \lambda_{0}}{d q_{0}}\left(q_{L}-\frac{s_{0}}{\lambda_{0}}\left(x_{L}-x_{0}\right)\right) \\
& +\frac{d}{d \lambda_{0}}\left(\frac{\eta_{L}}{\eta_{R}+\eta_{L}}\right) \frac{d \lambda_{0}}{d q_{0}}\left(q_{R}-\frac{s_{0}}{\lambda_{0}}\left(x_{R}-x_{0}\right)\right) \\
& +\frac{\eta_{R}}{\eta_{R}-\eta_{L}}\left(x_{L}-x_{0}\right) \frac{d}{d q_{0}}\left(\frac{s_{0}}{\lambda_{0}}\right) \\
& -\frac{\eta_{L}}{\eta_{R}-\eta_{L}}\left(x_{R}-x_{0}\right) \frac{d}{d q_{0}}\left(\frac{s_{0}}{\lambda_{0}}\right) \\
& q_{0}^{n+1}=q_{0}^{n}-\frac{\hat{q}}{\frac{d \hat{q}}{d q_{0}}}
\end{aligned}
$$

The solution of Eq. 18 can be deferred until a converged value for $q_{0}$ is obtained from Eq. 19. The reconstructed interface can now be computed.

$$
(f-h)_{0}=f\left(q_{0}\right)-\nu_{0}\left(\frac{d q}{d x}\right)_{0}
$$




\section{Discretization Considerations}

The transformed variable $\eta$ from Eq. 7 is a function of Reynolds number $\operatorname{Re} x=\frac{\lambda_{0}\left(x-x_{0}\right)}{\nu_{0}}$.

$$
\eta=\frac{\left(x-x_{0}\right)}{\operatorname{Rex}}(\exp [\operatorname{Re} x]-1)
$$

The cell Reynolds numbers $\left(\operatorname{Rex}_{L}\right.$ and $\left.R e x_{R}\right)$ provide a metric for resolution of diffusion dominated structures (boundary layers, shear layers, viscous shocks) across or within a cell. In most circumstances, the cell Reynolds number is of order 1 to 10 across boundary layers and shear layers but can grow many orders of magnitude larger in "inviscid flow" domains (ie. regions where viscosity effects on the flow structure are vanishingly small). When $R e x_{L} \gg 1$ (wave speed negative) and $\operatorname{Re} x_{R} \ll-1$ the value of $q_{0}$ from Eq. 17 is defined by the upstream conditions at $x_{R}$. Conversely, when $\operatorname{Rex}_{R} \gg 1$ (wave speed positive) and $\operatorname{Re} x_{L} \ll-1$ the value of $q_{0}$ from Eq. 17 is defined by the upstream conditions at $x_{L}$. In numerical implementations the maximum value of $|R e x|$ is set to 100 to eliminate occurrences of numeric overflow or underflow in the evaluation of $\eta$.

A special case must be considered when the converged wave speed $\lambda_{0}=0$, even beyond implementation of the proper limiting forms in Eqs. 8 and 13. A shock is formed when $\lambda_{L}>0$ and $\lambda_{R}<0$. If the shock is not resolved $\left(\left|\operatorname{Re} x_{L}\right|>1\right.$ and $\left.\left|\operatorname{Re} x_{R}\right|>1\right)$ and if $\lambda_{0}=0$ then the interface value of $q_{0}$ is a linear average of the left and right states and the gradient $\left(\frac{d q}{d x}\right)_{0}$ is a divided difference between the right and left states. This resulting approximation is extremely poor because the transition from $q_{R}$ to $q_{L}$ through $q_{0}$ takes place over a distance $\left|x_{R s}-x_{L s}\right| \approx \frac{\nu_{0}}{\lambda_{R}-\lambda_{L} \mid}$ which is a factor $\left|\operatorname{Re}\left(x_{R}-x_{L}\right)\right|$ less than the distance $\left|x_{R}-x_{L}\right|$. Without any special consideration of this shortcoming, the limiting form of the expressions for $q_{0}$ (Eq. 17) and $\left(\frac{d q}{d x}\right)_{0}$ (Eq. 18) becomes

$$
\begin{aligned}
q_{0} & =\frac{\left(x_{R}-x_{0}\right)\left(q_{L}-\frac{s_{0}}{2 \nu_{0}}\left(x_{L}-x_{0}\right)^{2}\right)-\left(x_{L}-x_{0}\right)\left(q_{R}-\frac{s_{0}}{2 \nu_{0}}\left(x_{R}-x_{0}\right)^{2}\right)}{x_{R}-x_{L}} \\
\left(\frac{d q}{d x}\right)_{0} & =\frac{\left(q_{L}-\frac{s_{0}}{2 \nu_{0}}\left(x_{L}-x_{0}\right)^{2}\right)-\left(q_{R}-\frac{s_{0}}{2 \nu_{0}}\left(x_{R}-x_{0}\right)^{2}\right)}{x_{L}-x_{R}}
\end{aligned}
$$

The expression for $\left(\frac{d q}{d x}\right)_{0}$ in Eq. 23 may be modified by replacing values of $x_{L}$ and $x_{R}$ with $x_{L s}$ and $x_{R s}$ respectively to account for the thin transition domain.

$$
\begin{aligned}
x_{R s}-x_{L s} & =\frac{\nu_{0}}{\left|\lambda_{R}-\lambda_{L}\right|} \\
x_{L s} & =x_{0}-\frac{\left(x_{R s}-x_{L s}\right)}{2} \\
x_{R s} & =x_{0}+\frac{\left(x_{L s}-x_{L s}\right)}{2} \\
\left(\frac{d q}{d x}\right)_{0} & =\frac{\left(q_{L}-\frac{s_{0}}{2 \nu_{0}}\left(x_{L s}-x_{0}\right)^{2}\right)-\left(q_{R}-\frac{s_{0}}{2 \nu_{0}}\left(x_{R s}-x_{0}\right)^{2}\right)}{x_{L s}-x_{R s}}
\end{aligned}
$$

Note that this special case arises because the wave speed at $x_{0}$ converged exactly to 0 . Had $\lambda_{0}$ been initialized with a non-zero value resulting in a large value for either $\eta_{R}$ or $\eta_{L}$ then the converged interface value would approach the left or right state, respectively (see Eq. 17). In either case, the high gradient transition zone would occur at the opposite end of the interval, away from $x_{0}$. This observation suggests related formulations for the analytic solution that avoids this difficulty. Some of these, dealing with attempts to better resolve wave speed across the cell, are presented in Appendix A. The next formulation is a simpler approach. 


\section{B. End-Point Wave Speed Approximation (EPWS)}

\section{Nominal Algorithm}

In the constant wave speed (CWS) approximation of Sec. A the analytic solution to a single linearized equation centered at interface $x_{0}$ (Eq. 6) forms the basis for reconstruction of the inviscid and viscous flux. The reconstruction algorithm required a Newton iteration to accommodate the implicit dependence of wave speed $\lambda_{0}$ on $q_{0}$. It also required a modification to account for a special case in which the wave speed equals zero within a shock. Both of these difficulties can be overcome if we reformulate on the basis of two linearized equations referenced to the right and left states of the interval. The origin is retained at the interface in this End-Point Wave Speed (EPWS) formulation.

$$
\begin{aligned}
\lambda_{L} \frac{d q}{d x} & =\nu_{L} \frac{d^{2} q}{d x^{2}}+s_{L 0} \\
\lambda_{R} \frac{d q}{d x} & =\nu_{R} \frac{d^{2} q}{d x^{2}}+s_{R 0}
\end{aligned}
$$

The source terms carry a dual subscript $L 0$ and $R 0$ to acknowledge that algebraic contributions are taken from the appropriate right or left state while gradient contributions will carry a smaller stencil if evaluated at the interface $x_{0}$. Transformed coordinates $\alpha(x)$ and $\beta(x)$ are introduced to take on the same role as $\eta(x)$ used in the constant wave speed approximation (Eq. 7).

$$
\begin{aligned}
\alpha(x) & =\int_{0}^{x-x_{0}} \exp \left[\frac{\lambda_{L}\left(x^{\prime}\right)}{\nu_{L}}\right] d x^{\prime} & \beta(x) & =\int_{0}^{x-x_{0}} \exp \left[\frac{\lambda_{R}\left(x^{\prime}\right)}{\nu_{R}}\right] d x^{\prime} \\
& =\frac{\nu_{L}}{\lambda_{L}}\left(\exp \left[\frac{\lambda_{L}\left(x-x_{0}\right)}{\nu_{L}}\right]-1\right) & & =\frac{\nu_{R}}{\lambda_{R}}\left(\exp \left[\frac{\lambda_{R}\left(x-x_{0}\right)}{\nu_{R}}\right]-1\right) \\
\frac{d \alpha}{d x} & =\exp \left[\frac{\lambda_{L}\left(x-x_{0}\right)}{\nu_{L}}\right] & \frac{d \beta}{d x} & =\exp \left[\frac{\lambda_{R}\left(x-x_{0}\right)}{\nu_{R}}\right] \\
\frac{d^{2} \alpha}{d x^{2}} & =\frac{\lambda_{L}}{\nu_{L}} \frac{d \alpha}{d x} & \frac{d^{2} \beta}{d x^{2}} & =\frac{\lambda_{R}}{\nu_{R}} \frac{d \alpha}{d x}
\end{aligned}
$$

Evaluation of limiting forms as $\lambda_{L} \rightarrow 0$ or $\lambda_{R} \rightarrow 0$ as well as the general and particular solutions to Eqs. 28 and 29 proceed exactly as in the previous section. The left and right state reference solutions are:

$$
\begin{aligned}
q_{0, \alpha} & =\frac{\alpha_{R}\left(q_{L}-\frac{s_{L 0}}{\lambda_{L}}\left(x_{L}-x_{0}\right)\right)-\alpha_{L}\left(q_{R}-\frac{s_{L 0}}{\lambda_{L}}\left(x_{R}-x_{0}\right)\right)}{\alpha_{R}-\alpha_{L}} \\
q_{0, \beta} & =\frac{\beta_{R}\left(q_{L}-\frac{s_{R 0}}{\lambda_{R}}\left(x_{L}-x_{0}\right)\right)-\beta_{L}\left(q_{R}-\frac{s_{R 0}}{\lambda_{R}}\left(x_{R}-x_{0}\right)\right)}{\beta_{R}-\beta_{L}} \\
\left(\frac{d q}{d x}\right)_{0, \alpha} & =\frac{\left(q_{L}-\frac{s_{L 0}}{\lambda_{L}}\left(x_{L}-\alpha_{L}\right)\right)-\left(q_{R}-\frac{s_{L 0}}{\lambda_{L}}\left(x_{R}-\alpha_{R}\right)\right)}{\alpha_{L}-\alpha_{R}} \\
\left(\frac{d q}{d x}\right)_{0, \beta} & =\frac{\left(q_{L}-\frac{s_{R 0}}{\lambda_{R}}\left(x_{L}-\beta_{L}\right)\right)-\left(q_{R}-\frac{s_{R 0}}{\lambda_{R}}\left(x_{R}-\beta_{R}\right)\right)}{\beta_{L}-\beta_{R}}
\end{aligned}
$$

If $\lambda_{L}=0$ then:

$$
\begin{aligned}
q_{0, \alpha} & =\frac{\left(x_{R}-x_{0}\right)\left(q_{L}-\frac{s_{L 0}}{2 \nu_{L}}\left(x_{L}-x_{0}\right)^{2}\right)-\left(x_{L}-x_{0}\right)\left(q_{R}-\frac{s_{L 0}}{2 \nu_{L}}\left(x_{R}-x_{0}\right)^{2}\right)}{x_{R}-x_{L}} \\
\left(\frac{d q}{d x}\right)_{0, \alpha} & =\frac{\left(q_{L}-\frac{s_{L 0}}{2 \nu_{L}}\left(x_{L}-x_{0}\right)^{2}\right)-\left(q_{R}-\frac{s_{L 0}}{2 \nu_{L}}\left(x_{R}-x_{0}\right)^{2}\right)}{x_{L}-x_{R}}
\end{aligned}
$$


If $\lambda_{R}=0$ then:

$$
\begin{aligned}
q_{0, \beta} & =\frac{\left(x_{R}-x_{0}\right)\left(q_{L}-\frac{s_{R 0}}{2 \nu_{R}}\left(x_{L}-x_{0}\right)^{2}\right)-\left(x_{L}-x_{0}\right)\left(q_{R}-\frac{s_{R 0}}{2 \nu_{R}}\left(x_{R}-x_{0}\right)^{2}\right)}{x_{R}-x_{L}} \\
\left(\frac{d q}{d x}\right)_{0, \beta} & =\frac{\left(q_{L}-\frac{s_{R 0}}{2 \nu_{R}}\left(x_{L}-x_{0}\right)^{2}\right)-\left(q_{R}-\frac{s_{R 0}}{2 \nu_{R}}\left(x_{R}-x_{0}\right)^{2}\right)}{x_{L}-x_{R}}
\end{aligned}
$$

In the usual case with $x_{0}=\left(x_{L}+x_{R}\right) / 2$ expressions for $q_{0}$ and $\left(\frac{d q}{d x}\right)_{0}$ are simplified as follows. First define local cell Reynolds numbers.

$$
\begin{aligned}
& R e_{L}=\frac{\lambda_{L}\left(x_{R}-x_{L}\right)}{\nu_{L}} \\
& R e_{R}=\frac{\lambda_{R}\left(x_{L}-x_{R}\right)}{\nu_{R}}
\end{aligned}
$$

Next, define reconstruction factors as a function of local cell Reynolds numbers.

$$
\begin{aligned}
& r_{f M 0}=\frac{1}{\exp \left(\frac{R e_{M}}{2}\right)+1} \\
& r_{f M 1}=\frac{\exp \left(\frac{R e_{M}}{2}\right) R e_{M}}{\exp \left(\operatorname{Re}_{M}\right)-1}
\end{aligned}
$$

with subscript $M$ referring to the left or right node as needed. The interface values as approximated from the left and right states are now more simply expressed.

$$
\begin{aligned}
q_{0, \alpha} & =q_{L}+\left(q_{R}-q_{L}\right) r_{f L 0}+\frac{s_{L 0}\left(x_{R}-x_{L}\right)}{\lambda_{L}}\left(\frac{1}{2}-r_{f L 0}\right) \\
q_{0, \beta} & =q_{R}+\left(q_{L}-q_{R}\right) r_{f R 0}+\frac{s_{R 0}\left(x_{L}-x_{R}\right)}{\lambda_{R}}\left(\frac{1}{2}-r_{f R 0}\right) \\
\left(\frac{d q}{d x}\right)_{0, \alpha} & =\frac{q_{R}-q_{L}}{x_{R}-x_{L}} r_{f L 1}+\frac{s_{L 0}}{\lambda_{L}}\left(1-r_{f L 1}\right) \\
\left(\frac{d q}{d x}\right)_{0, \beta} & =\frac{q_{R}-q_{L}}{x_{R}-x_{L}} r_{f R 1}+\frac{s_{R 0}}{\lambda_{R}}\left(1-r_{f R 1}\right)
\end{aligned}
$$

The reconstruction factors are bounded between 0 and 1.The limiting forms for $q_{0}$ and $\left(\frac{d q}{d x}\right)_{0}$ as $\lambda \rightarrow 0$ for either node are written

$$
\begin{array}{rlrl}
q_{0, \alpha} & =\frac{1}{2}\left(q_{R}+q_{L}\right)-\frac{s_{L 0}\left(x_{R}-x_{L}\right)^{2}}{8 \nu_{L}} & \text { for } & \lambda_{L} \rightarrow 0 \\
q_{0, \beta} & =\frac{1}{2}\left(q_{R}+q_{L}\right)-\frac{s_{R 0}\left(x_{R}-x_{L}\right)^{2}}{8 \nu_{R}} & \text { for } \lambda_{R} \rightarrow 0 \\
\left(\frac{d q}{d x}\right)_{0, \alpha} & =\frac{q_{R}-q_{L}}{x_{R}-x_{L}} & \text { for } \lambda_{L} \rightarrow 0 \\
\left(\frac{d q}{d x}\right)_{0, \beta} & =\frac{q_{R}-q_{L}}{x_{R}-x_{L}} & \text { for } \lambda_{R} \rightarrow 0
\end{array}
$$

An arithmetic average of the left and right reference state solution for flux is defined.

$$
\begin{aligned}
f_{0} & =\frac{f\left(q_{0, \alpha}\right)+f\left(q_{0, \beta}\right)}{2} \\
h_{0} & =\frac{\left(\nu \frac{d q}{d x}\right)_{0, \alpha}+\left(\nu \frac{d q}{d x}\right)_{0, \beta}}{2}
\end{aligned}
$$

Equations 43 and 44 explicitly define the interface flux and do not require the Newton iterations required in the constant wave speed formulation of the previous section. 


\section{Discretization Considerations}

Numerical experiments described in the next section have been the primary basis for assessing numerical stability and accuracy. In these experiments, all implemented with implicit line relaxation, the simplest method for insuring stability is to guarantee that the local cell Reynolds numbers never exceed a specified maximum value. This limit is implemented by first defining a reference cell Reynolds number for the interval.

$$
\bar{R} e=\frac{\max \left(\left|\lambda_{L}\right|,\left|\lambda_{R}\right|\right)\left(\left|x_{R}-x_{L}\right|\right)}{\min \left(\nu_{L}, \nu_{R}\right)}
$$

A maximum value for the reference cell Reynolds number $\bar{R} e_{\max }$ is specified and diffusivity at the left and right states is reset.

$$
\begin{aligned}
& \nu_{L} \leftarrow \nu_{L} \max \left(1, \frac{\phi \bar{R} e}{\bar{R} e_{\max }}\right) \\
& \nu_{R} \leftarrow \quad \nu_{R} \max \left(1, \frac{\phi \bar{R} e}{\bar{R} e_{\max }}\right)
\end{aligned}
$$

where $\phi$ is a limiter bounded by 0 (no artificial diffusivity) and 1 (maximum artificial diffusivity). Numerical tests show that $\bar{R} e_{\max }=8$ does not engage the limiter for any reasonably resolved viscous layer and will result in a near perfect jump across the cell if the layer is unresolved. The limiting enhances stability for cases of poorly resolved boundary layers and shocks.

Artificially increasing the diffusivity to constrain the reference cell Reynolds number from exceeding $\bar{R} e_{\max }$ everywhere $(\phi=1)$ provides acceptable solutions to Burger's equation. However, such an algorithm will undoubtedly corrupt a Navier-Stokes simulation with moderate to high vehicle Reynolds number because there is no possibility to maintain a cell Reynolds number less than 8 across the entire boundary layer without introducing an unacceptably large number of mesh points. The net effect would act much the same as adding turbulent diffusivity to the solution, thickening the boundary layer and moderating the gradients. Numerical experiments using line-implicit relaxation (next section) have been conducted to test algorithms designed to circumvent this problem. The tests reveal that restrictions on the cell Reynolds number are only required in the vicinity of a sign change in the eigenvalue (wave speed) from the left to right node. In the case of diverging waves (both directed away from the interface) a cell Reynolds number less than 8 is required to prevent the formation of an expansion shock - dissipation is the only process that connects the left and right states. In the case of converging waves (both directed toward the interface) the cell Reynolds number restriction is required to maintain stability and prevent formation of overshoots and undershoots across a shock.

Conceptually, one would like to engage the requisite artificial viscosity only at interfaces where the product $\lambda_{L} \lambda_{R}<0$. Numerical tests show that a ramp in artificial diffusivity factor is required from 0 to 1 in the neighborhood of the change in eigenvalue in order to achieve convergence. The neighborhood of a change in eigenvalue is defined as any interval in which the product $\left(\lambda_{L}+\omega\left(\lambda_{L}-\right.\right.$ $\left.\left.\lambda_{R}\right)\right)\left(\lambda_{R}+\omega\left(\lambda_{R}-\lambda_{L}\right)\right)<0$. The factors represent a linearly extrapolated eigenvalue located $\omega$ cells to the left and right of the respective end points. A value $\omega=1$ is recommended. Larger values of $\omega$ tend to diffuse the shock over more cells but have beneficial effects on stability. The limiter $\phi$ for engaging extra diffusivity in the neighborhood of a change in eigenvalue is now defined

$$
\begin{aligned}
\phi & =3 \bar{\phi}^{2}-2 \bar{\phi}^{3} \\
\bar{\phi} & \left.=\max \left[0,\left(\left(\omega^{2}+\omega\right)-\left(1+2 \omega+2 \omega^{2}\right) \frac{\lambda_{L} \lambda_{R}}{\lambda_{L}^{2}+\lambda_{R}^{2}}\right)\right) /\left(2 \omega^{2}+2 \omega+\frac{1}{2}\right)\right]
\end{aligned}
$$

The variable $\bar{\phi}$ in Eq. 48 defines a normalized parameter varying between 0 and 1. A value of 0 indicates the interval is not in the neighborhood of a change in eigenvalue or that one of the 
extrapolated eigenvalues equals zero. A value of 1 indicates that the eigenvalues at the endpoints are equal magnitude but of opposite sign. The variable $\phi$ in Eq. 48 is defined such that $\phi(0)=0$, $\phi(1)=1, \frac{d \phi}{d \phi}(0)=0$, and $\frac{d \phi}{d \phi}(1)=0$.

Large values of $\left|R e_{M}\right|$ cause machine zero values of reconstruction factor $r_{f M 1}$ in Eq. 40 which in turn cause machine zero values of $\left(\frac{d q}{d x}\right)_{0}$ in Eq. 41 in the absence of source terms. Stability of the line relaxation is enhanced in these cases by resetting $r_{f M 1}$ to 1 in Eq. 41 in order to recover a conventional central difference representation of diffusive gradients across neighborhood of the change in eigenvalue. The transition from the semi-analytic gradient reconstruction to conventional central difference is implemented with the following change to the reconstruction factor $r_{f M 1}$.

$$
r_{f M 1, \text { blended }}=r_{f M 1}(1-\phi)+\phi
$$

This blended reconstruction factor may be used in place of $r_{f M 1}$ to evaluate the gradients in Eq. 41.

\section{Application - Burger's Equation}

Burger's equation, the simplest test including effects of both a diffusion term and a non-linear convective flux term, is defined in Eq. 50.

$$
\frac{\partial u}{\partial t}+\frac{1}{2} \frac{\partial u^{2}}{\partial x}=\nu \frac{\partial^{2} u}{\partial x^{2}}
$$

In terms of the generic scalar conservation law presented in Eq. 1 note that $q=u, f=\frac{u^{2}}{2}, h=\nu \frac{\partial u}{\partial x}$ and $s=0$. Two sets of boundary conditions are tested: the first with a centered shock and the second with end wall boundary layers. Wave speed is defined by $\lambda=\frac{d f}{d q}=u$.

\section{Center Shock}

The solution domain is defined across $[-1,1]$. Boundary conditions are fixed with $u(-1)=1$, $u(1)=-1$. The initial condition is defined $u(x)=-x$. Wave speeds are negative for $x>0$ and positive for $x<0$; consequently, waves carry the boundary values of $u$ to the center of the domain where dissipation effects smooth the transition from the left to right states. The exact solution for the steady state limit is defined in Eq. 51.

$$
u(x)=a \tanh \left(-\frac{a x}{2 \nu}\right)
$$

The coefficient $a$ is a function of $\nu$ defined with an implicit relation to satisfy the boundary conditions.

$$
a \tanh \left(\frac{a}{2 \nu}\right)=1
$$

Line relaxation is used with $\frac{\Delta x}{\Delta t}=0.1$ for the numerical solution of Eq. 51 with waves converging to the center of the domain.

\section{End Wall Boundary Layers}

The solution domain is defined across $[-1,1]$. Boundary conditions are fixed with $u(-1)=-1$, $u(1)=1$. The initial condition is defined $u(x)=x$. Wave speeds are positive for $x>0$ and negative for $x<0$; consequently, waves carry the center value of $u=0$ to the boundaries of the domain where dissipation effects smooth the transition to the end states. The exact solution for the steady state limit is defined in Eq. 53.

$$
u(x)=a \tan \left(\frac{a x}{2 \nu}\right)
$$


The coefficient $a$ is a function of $\nu$ defined with an implicit relation to satisfy the boundary conditions.

$$
a \tan \left(\frac{a}{2 \nu}\right)=1
$$

A constraint, $0<a<\pi \nu$, must be imposed to avoid solutions with discontinuities. Line relaxation is used with $\frac{\Delta x}{\Delta t}=0.1$ for the first 10 global relaxation steps of the numerical solution of Eq. 51 with waves diverging from the center of the domain. After iteration 10 the steady form of the equation is relaxed $\left(\frac{\Delta x}{\Delta t}=0\right)$. The converged solution takes a long time to set up as $\nu \rightarrow 0$ because wave speed goes to zero over the domain between the end wall boundary layers.

\section{Comparisons - Shock}

The numerical solution to Eq. 50 for the center shock boundary conditions using the constant wave speed (CWS) reconstruction is presented as a solid black line in Fig. 1(a) for $\nu=1$. and in Fig. 1(b) for $\nu=0.01$. There is no discernible difference between the results of the CWS formulation and the end point wave speed (EPWS) for $\nu=1$ on the scale of Fig. 1(a). The EPWS reconstruction uses $\phi=1$ (cell Reynolds number limiting engaged everywhere) unless otherwise noted. The shock front sharpens as $\nu \rightarrow 0$.

The broken lines show the difference between the semi-analytic formulations $u$ and the exact solution $u_{e}$ (Eq. 51) and between the reference formulation using Roes scheme with Yee's Symmetric Total Variation Diminishing (STVD) $u_{R o e}$ and $u_{e}$. For $\nu>0.01$ all formulations are in good agreement with the exact solution but the semi-analytic formulations are closer to the exact solution. (This trend will be confirmed in subsequent tables.)

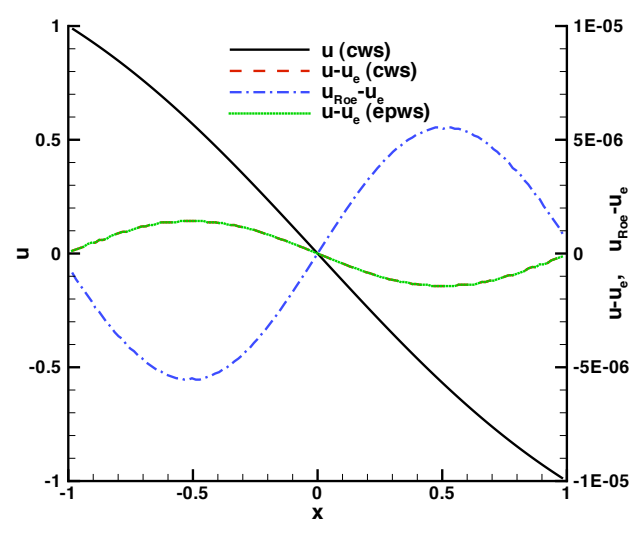

(a) $\nu=1.0$

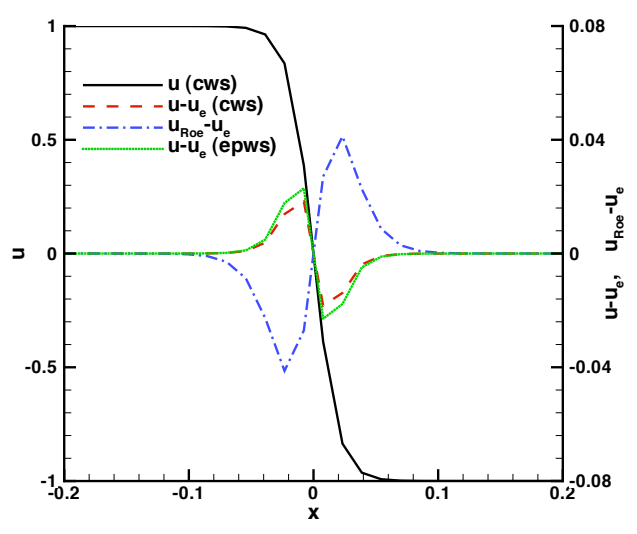

(b) $\nu=0.01$, note $\mathrm{x}$ scale change

Figure 1. Solution and error distribution for Burgers equation with 129 cells.

A larger view of the solution quality for a wide range of $\nu$ and cell number are summarized in Tables $1-6$. The error norm in column 3 is defined as

$$
\left|u-u_{e}\right|=\sum_{i=2}^{\text {cells }}\left|u_{i}-u_{e, i}\right| \Delta x
$$

The error norm in column 4 is defined similarly for the Roe formulation. Note that the Roe scheme error norm in column 4 is constant for the odd number of cells with small value of $\nu_{0}$ and cell Reynolds numbers greater than 100 in column 8. This result was double-checked and is caused by 
the rounding of the shock front at the single point to the right and left of the zero point such that the integrated norm is a constant. Column 5 shows the number of iterations required to converge the residual norm of the semi-analytic formulation to the value shown in column 6 . Column 7 shows the residual norm achieved by the reference Roe formulation in the same number of iterations. The last column shows the maximum cell Reynolds number achieved in any cell to provide a metric for the quality of resolution.

Tables 1 - 3 contain error norm results and convergence data for the CWS and EPWS formulations for an odd number of cells spanning the domain. Tables 4 - 6 contain the same data for an even number of cells. Dependent variables $u_{i}$ are situated at the end points of each cell; consequently, the odd number cell tests have adjacent nodes that span the shock for $\nu \rightarrow 0$. The even cell cases have a node in the center of the discontinuity. Both instances are recorded here to test effectiveness of the choice $\bar{R} e_{\max }=8$ used in Eq. 46 under extremes in shock jump end states. The CWS and EPWS formulations show significant improvement over the reference Roe formulation for all simulations with $\nu \geq 0.01$. The Roe's scheme has a cleaner shock jump than the CWS formulation for the odd number of cells and the EPWS formulation for even number of cells.

Note that Tables 3 and 6 contain the results for EPWS where the limiter on cell Reynolds number is only engaged in the vicinity of a change in sign of the eigenvalue across an interval. In this case $\omega=1$ and $\bar{R} e_{\max }=4$. The value of 4 comes from numerical experiments indicating a good robustness under line relaxation without introduction of excessive smearing of shocks. The error norms between the two versions of EPWS are equivalent when the cell Reynolds number (right column) is less than 4 . The globally engaged limiter $(\phi=1)$ gives a better result than the restricted limiter; however, the globally engaged limiter employed a value of $\bar{R} e_{\max }=8$ that gives a near perfect shock jump for the odd number cell cases. In all other tests involving shocks with even number of cells or boundary layers (next section) the restricted limiter gives better results than the global limiter in any case where the cell Reynolds number exceeds 4 .

\section{Comparisons - Boundary Layer}

The numerical solution to Eq. 50 for the end wall boundary layers using the constant wave speed (CWS) reconstruction is presented as a solid black line in Fig. 2(a) for $\nu=1.0$ and in Fig. 2(b) for $\nu=0.01$. There is no discernible difference between the results of the CWS formulation and the end point wave speed (EPWS) for $\nu=1$ on the scale of Fig. 2(a). Again, the EPWS reconstruction uses $\phi=1$ (cell Reynolds number limiting engaged everywhere) unless otherwise noted. The boundary layer steepens as $\nu \rightarrow 0$.

The broken lines show the difference between the semi-analytic formulations $u$ and the exact solution $u_{e}$ (Eq. 53) and between the reference formulation using Roe's scheme with Yee's Symmetric Total Variation Diminishing (STVD) $u_{\text {Roe }}$ and $u_{e}$. The maximum error for the $\nu=1.0$ case occurs away from the wall when the boundary layer is well resolved. In contrast, the maximum error occurs at the wall for $\nu=0.01$ as resolution of the high gradient region is decreased. For $\nu>0.01$ all formulations are in good agreement with the exact solution but the semi-analytic formulations are closer to the exact solution. (This trend is confirmed in subsequent tables.) In Fig. 2(b) with $\nu=0.01$ the semi-analytic formulations are still closer to the exact solution than the reference formulation. The exact solution for the gradient at the half cell location next to the boundary $x=-1$ in Fig. $2(\mathrm{~b})$ is $\left(\frac{d u}{d x}\right)_{e}=25.996$. The semi-analytic values for the gradient are

$\left(\frac{d u}{d x}\right)_{C W S}=26.372$ (1.44\% error) and $\left(\frac{d u}{d x}\right)_{E P W S}=26.069$ (0.28\% error). The central difference approximation used in the reference formulation gives $\left(\frac{d u}{d x}\right)_{r e f}=28.078$ (8.0\% error) .

In contrast to the shock case there is no significant difference between odd and even number of cells for error norms. Tables 7 for CWS reconstruction and 8 for the EPWS reconstruction show similar quality of agreement with the exact solution as observed in the shock solutions. Only for 
cell Reynolds numbers on the order of 10 to 1,000 does the reference scheme outperform the EPWS formulation. At Reynolds numbers higher then 2,000 the convergence of the reference scheme is unpredictable because no eigenvalue limiting (entropy fix) is applied. The Roe scheme diverged for some of the highest reynolds numbers because no eigenvalue limiting was engaged across the sonic line and the natural viscosity was not sufficient to prevent formation of a non-physical rarefaction shock.

Table 9 contains the results for EPWS where the limiter on cell Reynolds number is only engaged in the vicinity of a change in sign of the eigenvalue across an interval. In this case $\omega=1$ and $\bar{R} e_{\max }=4$ as discussed in the previous section. The error norms between the two versions of EPWS are equivalent when the cell Reynolds number (right column) is less than 4. The restricted limiter gives better results than the global limiter in any case where the cell Reynolds number exceeds 4 .

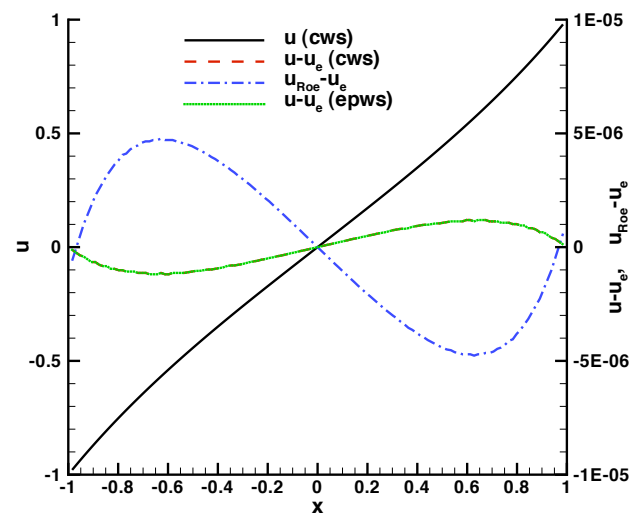

(a) $\nu=1.0$

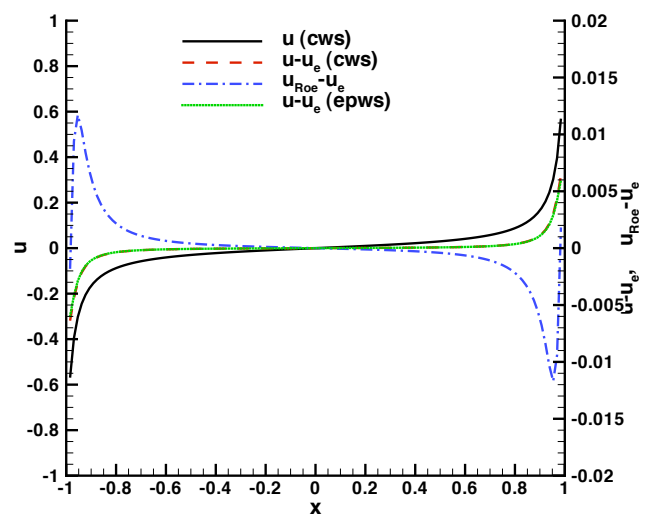

(b) $\nu=0.01$

Figure 2. Solution and error distribution for Burgers equation (boundary layer) with 129 cells. 


\section{One-Dimensional Vector Equation}

A one-dimensional, vector conservation law including both convection and diffusion is expressed by the following equation.

$$
\frac{\partial \mathbf{q}}{\partial t}+\frac{\partial}{\partial x}(\mathbf{f}-\mathbf{h})=\mathbf{s}
$$

A numerical solution to Eq. 56 written in conservative form must guarantee that the convective and diffusive flux exiting cell $i$ is exactly balanced by that entering cell $i+1$. Such a balance is enforced in the following difference equation.

$$
\frac{\mathbf{q}_{i}^{n+1}-\mathbf{q}_{i}^{n}}{\Delta t}+\frac{\left[(\mathbf{f}-\mathbf{h})_{i+1 / 2}-(\mathbf{f}-\mathbf{h})_{i-1 / 2}\right]}{\left(x_{i+1 / 2}-x_{i-1 / 2}\right)}=\mathbf{s}_{\mathbf{i}}
$$

An analytic solution to the linearized form of Eq. 56 will be used to define the convective and diffusive flux at cell interfaces $i \pm \frac{1}{2}$ in Eq. 57 .

$$
\frac{\partial \mathbf{q}}{\partial t}+\mathbf{A}_{0} \frac{\partial \mathbf{q}}{\partial x}=\nu_{0} \tilde{\mathbf{B}}_{0} \frac{\partial^{2} \tilde{\mathbf{q}}}{\partial x^{2}}+\mathbf{s}_{0}
$$

where $\mathbf{q}$ is the conserved variable set and $\tilde{\mathbf{q}}$ is the set of primitive variables defining the diffusion flux where $\mathbf{T} d \tilde{\mathbf{q}}=d \mathbf{q}$. Different strategies have been explored to diagonalize this system with various choices of artificial diffusion to remove the singularity from $\tilde{\mathbf{B}}$ and based on various choices for the primitive variable set $\tilde{\mathbf{q}}$. A characteristic variable formulation provides a foundation that maintains the physical wave speed and diagonal dominance (diagonal elements are all positive definite, off-diagonal elements are negative) in the linearization of the diffusion flux.

$$
\begin{gathered}
\frac{\partial \mathbf{q}}{\partial t}+\mathbf{A}_{0} \frac{\partial \mathbf{q}}{\partial x}=\nu_{0} \tilde{\mathbf{B}} \mathbf{T}^{-1} \mathbf{T} \frac{\partial^{2} \tilde{\mathbf{q}}}{\partial x^{2}}+\mathbf{s}_{0} \\
\frac{\partial \mathbf{q}}{\partial t}+\left(\mathbf{R} \mathbf{\Lambda} \mathbf{R}^{-1}\right)_{0} \frac{\partial \mathbf{q}}{\partial x}=\nu_{0}\left(\tilde{\mathbf{B}} \mathbf{T}^{-1}\right)_{0} \frac{\partial^{2} \mathbf{q}}{\partial x^{2}}+\mathbf{s}_{0} \\
\frac{\partial \mathbf{q}^{\prime}}{\partial t}+\boldsymbol{\Lambda}_{0} \frac{\partial \mathbf{q}^{\prime}}{\partial x}=\nu_{0}\left(\mathbf{R}^{-1} \tilde{\mathbf{B}} \mathbf{T}^{-1} \mathbf{R}\right)_{0} \frac{\partial^{2} \mathbf{q}^{\prime}}{\partial x^{2}}+\mathbf{R}^{-1}{ }_{0} \mathbf{s}_{0}
\end{gathered}
$$

The steady form of Eq. 59 is approximated as follows with $\Upsilon_{0}$ equal to the diagonal of $\left(\mathbf{R}^{-1} \tilde{\mathbf{B}} \mathbf{T}^{-1} \mathbf{R}\right)_{0}$ to simplify derivation of an analytic solution.

$$
\boldsymbol{\Lambda}_{0} \frac{d \mathbf{q}^{\prime}}{d x}=\nu_{0} \mathbf{\Upsilon}_{0} \frac{d^{2} \mathbf{q}^{\prime}}{d x^{2}}+\mathbf{R}^{-1}{ }_{0} \mathbf{s}_{0}+\nu_{0}\left(\mathbf{R}^{-1} \tilde{\mathbf{B}} \mathbf{T}^{-1} \mathbf{R}-\boldsymbol{\Upsilon}\right)_{0}\left(\frac{d^{2} \mathbf{q}^{\prime}}{d x^{2}}\right)_{0}
$$

The last term of Eq. 60 may be combined with the source term.

\section{A. End-Point Wave Speed (EPWS) - Nominal Algorithm for Systems}

The EPWS formulation of a semi-analytic solution will be developed here for systems of equations because it yields an explicitly defined interface. The solution expanded with respect to the left end point condition is developed in detail. The solution with respect to the right end point condition may then be inferred. The nomenclature closely follows that used in Sec. III - B.

The analytic solution to Eq. 60 using left state reference conditions is expressed.

$$
\mathbf{q}_{\alpha}^{\prime}=\mathbf{q}_{\alpha, 0}^{\prime}+\boldsymbol{\Psi}_{\alpha}\left(\frac{d \mathbf{q}^{\prime}}{d x}\right)_{\alpha, 0}+\boldsymbol{\Phi}_{\alpha} \mathbf{s}_{0 L}^{\prime}
$$


The elements of diagonal matrix $\boldsymbol{\Psi}_{\alpha}$ are defined as a function of the $j$ th element of $\boldsymbol{\Lambda}_{L}, \lambda_{L, j}$, and the $j$ th element of $\nu_{L} \boldsymbol{\Upsilon}_{L}, \nu_{L, j}$.

$$
\psi_{\alpha, j}=\left\{\begin{array}{cc}
\frac{\nu_{L, j}}{\lambda_{L, j}}\left(\exp \left[\frac{\lambda_{L, j}\left(x-x_{0}\right)}{\nu_{L, j}}\right]-1\right) & \lambda_{L, j} \neq 0 \\
x-x_{0} & \lambda_{L, j}=0
\end{array}\right.
$$

The elements of diagonal matrix $\boldsymbol{\Phi}_{\alpha}$ are defined

$$
\phi_{\alpha, j}=\left\{\begin{array}{cc}
\frac{1}{\lambda_{L, j}}\left[\left(x-x_{0}\right)-\psi_{\alpha, j}\right] & \lambda_{L, j} \neq 0 \\
-\frac{1}{2 \nu_{L, j}}\left(x-x_{0}\right)^{2} & \lambda_{L, j}=0
\end{array}\right.
$$

Conversion from characteristic variables $\mathbf{q}_{\alpha}^{\prime}$ to conserved variables $\mathbf{q}_{\alpha}$ follows.

$$
\begin{aligned}
\mathbf{R}_{L}^{-1}\left(\mathbf{q}_{\alpha}-\mathbf{q}_{\alpha, 0}\right) & =\boldsymbol{\Psi}_{\alpha} \mathbf{R}_{L}^{-1}\left(\frac{d \mathbf{q}}{d x}\right)_{\alpha, 0}+\boldsymbol{\Phi}_{\alpha} \mathbf{R}_{L}^{-1} \mathbf{s}_{0 L} \\
\mathbf{q}_{\alpha} & =\mathbf{q}_{\alpha, 0}+\mathbf{R}_{L} \boldsymbol{\Psi}_{\alpha} \mathbf{R}_{L}^{-1}\left(\frac{d \mathbf{q}}{d x}\right)_{\alpha, 0}+\mathbf{R}_{L} \boldsymbol{\Phi}_{\alpha} \mathbf{R}_{L}^{-1} \mathbf{s}_{0 L}
\end{aligned}
$$

The left and right values of $\mathbf{q}_{\alpha}$ are functions of $\boldsymbol{\Psi}_{\alpha}\left(x_{L}\right)=\boldsymbol{\Psi}_{\alpha, L}, \mathbf{\Psi}_{\alpha}\left(x_{R}\right)=\mathbf{\Psi}_{\alpha, R}, \boldsymbol{\Phi}_{\alpha}\left(x_{L}\right)=\boldsymbol{\Phi}_{\alpha, L}$ and $\boldsymbol{\Phi}_{\alpha}\left(x_{R}\right)=\boldsymbol{\Phi}_{\alpha, R}$.

$$
\begin{aligned}
& \mathbf{q}_{L}=\mathbf{q}_{\alpha, 0}+\mathbf{R}_{L} \boldsymbol{\Psi}_{\alpha, L} \mathbf{R}_{L}^{-1}\left(\frac{d \mathbf{q}}{d x}\right)_{\alpha, 0}+\mathbf{R}_{L} \boldsymbol{\Phi}_{\alpha, L} \mathbf{R}_{L}^{-1} \mathbf{s}_{0 L} \\
& \mathbf{q}_{R}=\mathbf{q}_{\alpha, 0}+\mathbf{R}_{L} \boldsymbol{\Psi}_{\alpha, R} \mathbf{R}_{L}^{-1}\left(\frac{d \mathbf{q}}{d x}\right)_{\alpha, 0}+\mathbf{R}_{L} \boldsymbol{\Phi}_{\alpha, R} \mathbf{R}_{L}^{-1} \mathbf{s}_{0 L}
\end{aligned}
$$

The interface conditions for $\mathbf{q}_{\alpha, 0}$ and $\left(\frac{d \mathbf{q}}{d x}\right)_{\alpha, 0}$ can be solved from Eqs. 66 and 67 .

$$
\begin{aligned}
& \left(\frac{d \mathbf{q}}{d x}\right)_{\alpha, 0}=\mathbf{R}_{L}\left(\boldsymbol{\Psi}_{\alpha, L}-\boldsymbol{\Psi}_{\alpha, R}\right)^{-1} \\
& {\left[\mathbf{R}_{L}^{-1}\left(\mathbf{q}_{L}-\mathbf{q}_{R}\right)-\left(\boldsymbol{\Phi}_{\alpha, L}-\boldsymbol{\Phi}_{\alpha, R}\right) \mathbf{R}_{L}^{-1} \mathbf{s}_{0 L}\right] } \\
\mathbf{q}_{\alpha, 0}= & \mathbf{R}_{L}\left(\boldsymbol{\Psi}_{\alpha, R}-\boldsymbol{\Psi}_{\alpha, L}\right)^{-1} \\
& {\left[\boldsymbol{\Psi}_{\alpha, R} \mathbf{R}_{L}^{-1} \mathbf{q}_{L}-\boldsymbol{\Psi}_{\alpha, L} \mathbf{R}_{L}^{-1} \mathbf{q}_{R}-\left(\boldsymbol{\Psi}_{\alpha, R} \boldsymbol{\Phi}_{\alpha, L}-\boldsymbol{\Psi}_{\alpha, L} \boldsymbol{\Phi}_{\alpha, R}\right) \mathbf{R}_{L}^{-1} \mathbf{s}_{0 L}\right] }
\end{aligned}
$$

The right end point expansion follows the same pattern as the left. The elements of diagonal matrix $\boldsymbol{\Psi}_{\beta}$ are defined

$$
\psi_{\beta, j}=\left\{\begin{array}{cc}
\frac{\nu_{R, j}}{\lambda_{R, j}}\left(\exp \left[\frac{\lambda_{R, j}\left(x-x_{0}\right)}{\nu_{R, j}}\right]-1\right) & \lambda_{R, j} \neq 0 \\
x-x_{0} & \lambda_{R, j}=0
\end{array}\right.
$$

The elements of diagonal matrix $\boldsymbol{\Phi}_{\beta}$ are defined

$$
\phi_{\beta, j}=\left\{\begin{array}{cc}
\frac{1}{\lambda_{R, j}}\left[\left(x-x_{0}\right)-\psi_{\beta, j}\right] & \lambda_{R, j} \neq 0 \\
-\frac{1}{2 \nu_{R, j}}\left(x-x_{0}\right)^{2} & \lambda_{R, j}=0
\end{array}\right.
$$

The interface conditions for $\mathbf{q}_{\beta, 0}$ and $\left(\frac{d \mathbf{q}}{d x}\right)_{\beta, 0}$ are defined.

$$
\begin{aligned}
\left(\frac{d \mathbf{q}}{d x}\right)_{\beta, 0}= & \mathbf{R}_{R}\left(\mathbf{\Psi}_{\beta, L}-\mathbf{\Psi}_{\beta, R}\right)^{-1} \\
& {\left[\mathbf{R}_{R}^{-1}\left(\mathbf{q}_{L}-\mathbf{q}_{R}\right)-\left(\mathbf{\Phi}_{\beta, L}-\boldsymbol{\Phi}_{\beta, R}\right) \mathbf{R}_{R}^{-1} \mathbf{s}_{0 R}\right] }
\end{aligned}
$$




$$
\begin{aligned}
\mathbf{q}_{\beta, 0}= & \mathbf{R}_{R}\left(\boldsymbol{\Psi}_{\beta, R}-\boldsymbol{\Psi}_{\beta, L}\right)^{-1} \\
& {\left[\boldsymbol{\Psi}_{\beta, R} \mathbf{R}_{R}^{-1} \mathbf{q}_{L}-\boldsymbol{\Psi}_{\beta, L} \mathbf{R}_{R}^{-1} \mathbf{q}_{R}-\left(\boldsymbol{\Psi}_{\beta, R} \boldsymbol{\Phi}_{\beta, L}-\boldsymbol{\Psi}_{\beta, L} \boldsymbol{\Phi}_{\beta, R}\right) \mathbf{R}_{R}^{-1} \mathbf{s}_{0 R}\right] }
\end{aligned}
$$

Additional simplifications expressed below (equivalent to scalar equation Eq. 41) can be introduced if one assumes $x_{0}=\frac{1}{2}\left(x_{R}+x_{L}\right)$.

$$
\begin{aligned}
& \mathbf{q}_{\alpha, 0}=\mathbf{q}_{L}+\mathbf{R}_{L} \mathbf{D}_{r f L 0} \mathbf{R}_{L}^{-1}\left(\mathbf{q}_{R}-\mathbf{q}_{L}\right)+\mathbf{R}_{L} \Lambda_{L}^{-1}\left(\frac{1}{2} \mathbf{I}-\mathbf{D}_{r f L 0}\right) \mathbf{R}_{L}^{-1}\left(x_{R}-x_{L}\right) \mathbf{s}_{0 L} \\
& \mathbf{q}_{\beta, 0}=\mathbf{q}_{R}+\mathbf{R}_{R} \mathbf{D}_{r f R 0} \mathbf{R}_{R}^{-1}\left(\mathbf{q}_{L}-\mathbf{q}_{R}\right)+\mathbf{R}_{R} \Lambda_{R}^{-1}\left(\frac{1}{2} \mathbf{I}-\mathbf{D}_{r f R 0}\right) \mathbf{R}_{R}^{-1}\left(x_{L}-x_{R}\right) \mathbf{s}_{0 R} \\
&\left(\frac{d \mathbf{q}}{d x}\right)_{\alpha, 0}=\mathbf{R}_{L} \mathbf{D}_{r f L 1} \mathbf{R}_{L}^{-1} \frac{\mathbf{q}_{R}-\mathbf{q}_{L}}{x_{R}-x_{L}}+\mathbf{R}_{L} \Lambda_{L}^{-1}\left(\mathbf{I}-\mathbf{D}_{r f L 1}\right) \mathbf{R}_{L}^{-1} \mathbf{s}_{0 L} \\
&\left(\frac{d \mathbf{q}}{d x}\right)_{\beta, 0}=\mathbf{R}_{R} \mathbf{D}_{r f R 1} \mathbf{R}_{R}^{-1} \frac{\mathbf{q}_{L}-\mathbf{q}_{R}}{x_{L}-x_{R}}+\mathbf{R}_{R} \Lambda_{R}^{-1}\left(\mathbf{I}-\mathbf{D}_{r f R 1}\right) \mathbf{R}_{R}^{-1} \mathbf{s}_{0 R}
\end{aligned}
$$

where $\mathbf{D}_{r f M 0}$ and $\mathbf{D}_{r f M 1}$ are diagonal matrices of reconstruction factors whose elements are bounded between 0 and 1 (with $M$ a dummy variable for $R$ or $L$ ) corresponding to scalar equation Eq. 40 whose elements are defined

$$
\begin{gathered}
d_{r f M 0, j}=\frac{1}{\exp \left(\frac{R e_{M, j}}{2}\right)+1} \\
d_{r f M 1, j}=\frac{\exp \left(\frac{R e_{M, j}}{2}\right) R e_{M, j}}{\exp \left(R e_{M, j}\right)-1}
\end{gathered}
$$

and

$$
\begin{aligned}
R e_{L, j} & =\frac{\lambda_{L, j}\left(x_{R}-x_{L}\right)}{v_{L, j}} \\
R e_{R, j} & =\frac{\lambda_{R, j}\left(x_{L}-x_{R}\right)}{v_{R, j}} .
\end{aligned}
$$

Note that $v_{M, j}$ is the $j$ th element of diagonal matrix $\nu_{M} \mathbf{\Upsilon}_{M}$ and $\lambda_{M, j}$ is the $j$ th element of diagonal matrix of eigenvalues $\Lambda_{M}$.

The limiting forms for the $j$ th diagonal elements when $\lambda_{j} \rightarrow 0$ in Eq. 74 are defined

$$
\begin{array}{ccccc}
d_{r f M 0, j} & = & \frac{1}{2} & \text { for } & \lambda_{M, j}=0 \\
\frac{x_{R}-x_{L}}{\lambda_{L, j}}\left(\frac{1}{2}-d_{r f L 0, j}\right) & = & -\frac{\left(x_{R}-x_{L}\right)^{2}}{8 v_{L, j}} & \text { for } & \lambda_{L, j}=0 \\
\frac{x_{L}-x_{R}}{\lambda_{R, j}}\left(\frac{1}{2}-d_{r f R 0, j}\right) & = & -\frac{\left(x_{L}-x_{R}\right)^{2}}{8 v_{R, j}} & \text { for } & \lambda_{R, j}=0 \\
d_{r f M 1, j} & = & 1 & \text { for } & \lambda_{M, j}=0 \\
\frac{1}{\lambda_{M, j}}\left(1-d_{r f M 1, j}\right) & = & 0 & \text { for } \lambda_{M, j}=0 .
\end{array}
$$

\section{B. Discretization Considerations for Systems of Equations}

A limited value of diffusivity is required to maintain a robust capture across shocks and expansions where an eigenvalue passes through zero. The limiter is based on a maximum value of a reference cell Reynolds number $\bar{R} e_{\max }$. The maximum is set to 8 if the limiter is applied globally as discussed earlier for Eq. 46. The maximum is set equal to 4 if the limiting is introduced only in the vicinity of a change in sign of eigenvalue. The reference cell Reynolds number is a scalar value that is still defined with Eq. 45, where $|\lambda|$ is taken as the maximum norm of the diagonal matrix of eigenvalues.

$$
\bar{R} e=\frac{\max \left(\left|\lambda_{L}\right|,\left|\lambda_{R}\right|\right)\left(\left|x_{R}-x_{L}\right|\right)}{\min \left(\nu_{L}, \nu_{R}\right)}
$$


The diffusivity is reset as originally discussed in Eq. 46.

$$
\begin{aligned}
& \nu_{L} \leftarrow \nu_{L} \max \left(1, \frac{\phi \overline{R e}}{\bar{R} e_{\max }}\right) \\
& \nu_{R} \leftarrow \nu_{R} \max \left(1, \frac{\phi R e}{R e_{\max }}\right)
\end{aligned}
$$

where $\phi$ is a limiter bounded by 0 (no artificial diffusivity) and 1 (maximum artificial diffusivity) as originally presented in Eqs. 48. Equation 48 is slightly modified below in order to emphasize that one is searching for a maximum over all $j$ elements of the matrix of eigenvalues.

$$
\begin{aligned}
\phi & =3 \bar{\phi}^{2}-2 \bar{\phi}^{3} \\
\bar{\phi} & =\max \left\{0, \max \left[\left(\left(\omega^{2}+\omega\right)-\left(1+2 \omega+2 \omega^{2}\right) \frac{\lambda_{L, j} \lambda_{R, j}}{\lambda_{L, j}^{2}+\lambda_{R, j}^{2}}\right) /\left(2 \omega^{2}+2 \omega+\frac{1}{2}\right)\right]\right\}
\end{aligned}
$$

Finally, the transition from the semi-analytic gradient reconstruction to conventional central difference is implemented with the following change to the diagonal matrix of reconstruction factors $\mathbf{D}_{r f M 1}$ as presented earlier in scalar Eq. 49.

$$
\mathbf{D}_{r f M 1, \text { blended }}=(1-\phi) \mathbf{D}_{r f M 1}+\phi \mathbf{I}
$$

This blended reconstruction factor may be used in place of $\mathbf{D}_{r f M 1}$ to evaluate the gradients in Eq. 74 .

\section{Application - Quasi, One-Dimensional Nozzle}

The governing equations for quasi one-dimensional nozzle flow are expressed

$$
\frac{\partial \mathbf{q} A}{\partial t}+\frac{\partial}{\partial x}[(\mathbf{f}-\mathbf{h}) A]=\mathbf{s}
$$

where $A(x)$ is the area distribution of the nozzle and

$$
\begin{gathered}
\mathbf{f}=\left[\begin{array}{c}
\rho u \\
p+\rho u^{2} \\
\rho u H
\end{array}\right] \\
\mathbf{h}=\left[\begin{array}{c}
0 \\
\frac{4}{3} \mu \frac{\partial u}{\partial x} \\
\frac{4}{3} \mu u \frac{\partial u}{\partial x}+\frac{\mu C_{p}}{\operatorname{Pr}} \frac{\partial T}{\partial x}
\end{array}\right] \\
\mathbf{s}=\left[\begin{array}{c}
0 \\
p \frac{\partial A}{\partial x} \\
0
\end{array}\right]
\end{gathered}
$$

The conserved variable vector $\mathbf{q}$ and primitive variable vector $\tilde{\mathbf{q}}$ are defined

$$
\mathbf{q}=\left[\begin{array}{c}
\rho \\
\rho u \\
\rho E
\end{array}\right] \quad, \quad \tilde{\mathbf{q}}=\left[\begin{array}{c}
\rho \\
u \\
T
\end{array}\right]
$$

Eq. 81 may be rewritten to move all nozzle area terms to the source.

$$
\frac{\partial \mathbf{q}}{\partial t}+\mathbf{A} \frac{\partial \mathbf{q}}{\partial x}=\nu \tilde{\mathbf{B}} \frac{d^{2} \tilde{\mathbf{q}}}{d x^{2}}+\mathbf{s}
$$

19 of 52 
where the source term is now defined by

$$
\mathbf{s}=\left[\begin{array}{c}
-\rho u \frac{d \log A}{d x} \\
-\rho u^{2} \frac{d \log A}{d x} \\
-\rho u H \frac{d \log A}{d x}
\end{array}\right]
$$

and relations for a calorically perfect gas are assumed.

$$
\begin{aligned}
p & =\tilde{\beta} \rho e \\
e & =C_{v} T \\
\tilde{\beta} & =\gamma-1=C_{p} / C_{v}-1 \\
c^{2} & =\gamma p / \rho
\end{aligned}
$$

The linearization employs matrices $\mathbf{A}$ and $\tilde{\mathbf{B}}$ defined below.

$$
\begin{gathered}
\frac{d \mathbf{f}}{d \mathbf{q}}=\mathbf{A}=\left[\begin{array}{ccc}
0 & 1 & 0 \\
u^{2}\left(\frac{\tilde{\beta}}{2}-1\right) & u(2-\tilde{\beta}) & \tilde{\beta} \\
u\left(u^{2} \frac{\beta}{2}-H\right) & -\tilde{\beta} u^{2}+H & \tilde{\beta} u+u
\end{array}\right] \\
\frac{d \mathbf{h}}{d\left(\frac{d \tilde{\mathbf{q}}}{d x}\right)}=\nu \tilde{\mathbf{B}}=\nu\left[\begin{array}{ccc}
0 & 0 & 0 \\
0 & \frac{4}{3} \rho & 0 \\
0 & \frac{4}{3} \rho u & \rho \frac{C_{p}}{\operatorname{Pr}}
\end{array}\right]
\end{gathered}
$$

Transformations from primitive to conservative variables $(\mathbf{T} d \tilde{\mathbf{q}}=d \mathbf{q})$ and conservative to primitive $\left(\mathbf{T}^{-1} d \mathbf{q}=d \tilde{\mathbf{q}}\right)$ are defined

$$
\mathbf{T}=\left[\begin{array}{ccc}
1 & 0 & 0 \\
u & \rho & 0 \\
E & \rho u & \rho C_{v}
\end{array}\right] \quad \mathbf{T}^{-1}=\left[\begin{array}{ccc}
1 & 0 & 0 \\
-\frac{u}{\rho} & \frac{1}{\rho} & 0 \\
\frac{1}{\rho C_{v}}\left(\frac{u^{2}}{2}-e\right) & -\frac{u}{\rho C_{v}} & \frac{1}{\rho C_{v}}
\end{array}\right]
$$

Diagonalization of $\mathbf{A}=\mathbf{R} \mathbf{\Lambda} \mathbf{R}^{-1}$ is implemented with

$$
\begin{gathered}
\mathbf{R}=\frac{1}{2 c^{2}}\left[\begin{array}{ccc}
2 & 1 & 1 \\
2 u & u+c & u-c \\
u^{2} & H+c u & H-c u
\end{array}\right] \quad \mathbf{R}^{-1}=\left[\begin{array}{ccc}
c^{2}-\tilde{\beta} \frac{u^{2}}{2} & \tilde{\beta} u & -\tilde{\beta} \\
\tilde{\beta} \frac{u^{2}}{2}-u c & c-\tilde{\beta} u & \tilde{\beta} \\
\tilde{\beta} \frac{u^{2}}{2}+u c & -c-\tilde{\beta} u & \tilde{\beta}
\end{array}\right] \\
\boldsymbol{\Lambda}=\left[\begin{array}{ccc}
u & 0 & 0 \\
0 & u+c & 0 \\
0 & 0 & u-c
\end{array}\right]
\end{gathered}
$$

All matrices appearing in Eq. 60 are defined for the quasi one-dimensional nozzle problem. The product $\mathbf{R}^{-1} \tilde{\mathbf{B}} \mathbf{T}^{-1} \mathbf{R}$ and its diagonal $\boldsymbol{\Upsilon}$ in this equation are evaluated.

$$
\begin{aligned}
& \mathbf{R}^{-1} \tilde{\mathbf{B}} \mathbf{T}^{-1} \mathbf{R}=\frac{1}{2 \operatorname{Pr}}\left[\begin{array}{ccc}
2 & -\tilde{\beta} & -\tilde{\beta} \\
-2 & \tilde{\beta}+\frac{4 \operatorname{Pr}}{3} & \tilde{\beta}-\frac{4 \operatorname{Pr}}{3} \\
-2 & \tilde{\beta}-\frac{4 \operatorname{Pr}}{3} & \tilde{\beta}+\frac{4 \operatorname{Pr}}{3}
\end{array}\right] \\
& \Upsilon=\frac{1}{2 \operatorname{Pr}}\left[\begin{array}{ccc}
2 & 0 & 0 \\
0 & \tilde{\beta}+\frac{4 \mathrm{Pr}}{3} & 0 \\
0 & 0 & \tilde{\beta}+\frac{4 \operatorname{Pr}}{3}
\end{array}\right]
\end{aligned}
$$


Three nozzle flows are simulated with area ratios of 20, 200, and 2,000 and respective exit pressure to stagnation pressure ratios of 0.1, 0.01, and 0.001. The gas model (MKS units) uses ratio of specific heats $\gamma=1.4$, molecular weight $M_{w}=28.8$, Prandtl number $\operatorname{Pr}=0.72$, and Sutherland's law for viscosity,

$$
\mu(T)=0.00001458205 T^{3 / 2} /(T+110.333) .
$$

The baseline cases specify $T_{\text {stag }}=2,000$. and $p_{\text {stag }}=10^{6}$. The baseline specifications result in reference cell Reynolds numbers exceeding 8 everywhere - even with the finest grid. An off baseline case specifies $p_{\text {stag }}=10^{3}$ which brings reference cell reynolds numbers to order 1 or less in the vicinity of a captured shock in the diverging section of the nozzle.

The area distribution is defined in Fig. 3. It has zero slope at the entrance and exit to simplify formulation of extrapolated boundary conditions at $x= \pm 1$. (The change in characteristic variables across the boundaries are set to zero when associated eigenvalues direct waves out of the nozzle.) The exponent (4) provides a very smooth, slow variation of area at the throat even for the largest area ratio tests.

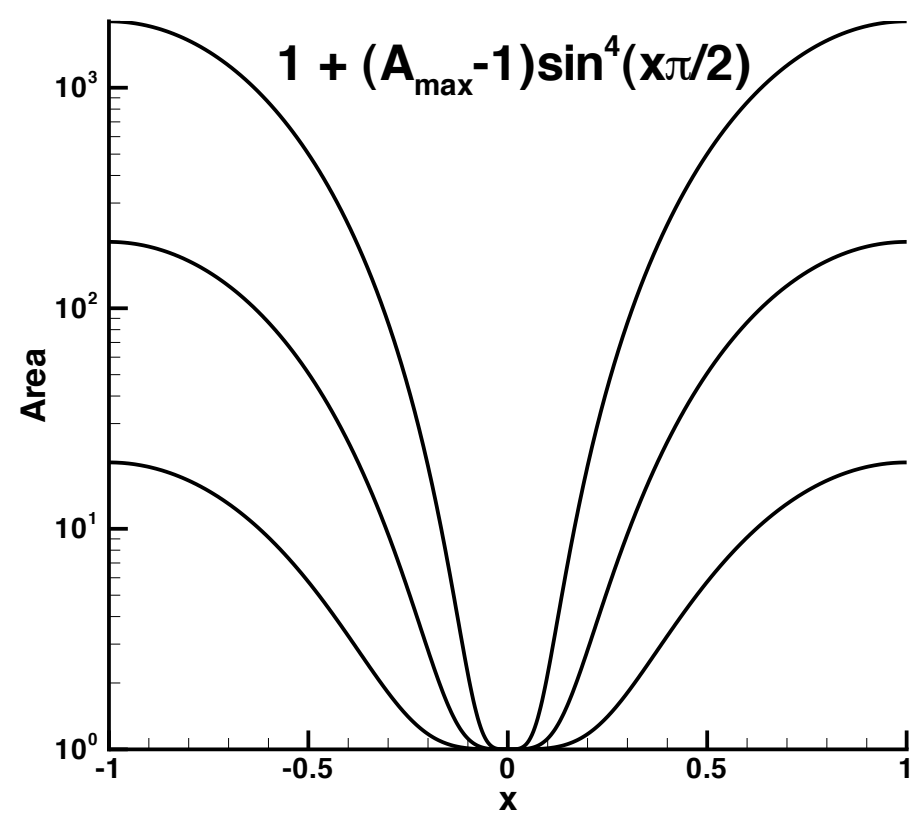

Figure 3. Area distributions used in quasi-one-dimensional nozzle tests.

At the subsonic inflow boundary

$$
\begin{aligned}
H_{1} & =H_{\text {stag }} \\
p_{1} \rho_{\text {stag }}^{\gamma} & =p_{\text {stag }} \rho_{1}^{\gamma} \\
R_{3,1}\left(\rho_{1}-\rho_{2}\right)+R_{3,2}\left((\rho u)_{1}-(\rho u)_{2}\right)+R_{3,3}\left((\rho E)_{1}-(\rho E)_{2}\right) & =0 .
\end{aligned}
$$

Exit pressures are specified to cause a standing shock in the diverging portion of the nozzle. Thus, 
at the subsonic outflow boundary

$$
\begin{aligned}
p_{N} & =p_{\text {exit }} \\
R_{1,1}\left(\rho_{N-1}-\rho_{N}\right)+R_{1,2}\left((\rho u)_{N-1}-(\rho u)_{N}\right)+R_{1,3}\left((\rho E)_{N-1}-(\rho E)_{N}\right) & =0 \\
R_{2,1}\left(\rho_{N-1}-\rho_{N}\right)+R_{2,2}\left((\rho u)_{N-1}-(\rho u)_{N}\right)+R_{2,3}\left((\rho E)_{N-1}-(\rho E)_{N}\right) & =0 .
\end{aligned}
$$

Implicit line relation is used to drive the residual of the discretized governing equations to zero.

$$
\frac{\left(\mathbf{q}_{i}^{n+1}-\mathbf{q}_{i}^{n}\right) A_{i}}{\Delta t_{i}}+\frac{\left[A_{i+1 / 2}(\mathbf{f}-\mathbf{h})_{i+1 / 2}-A_{i-1 / 2}(\mathbf{f}-\mathbf{h})_{i-1 / 2}\right]}{\left(x_{i+1 / 2}-x_{i-1 / 2}\right)}=\mathbf{s}_{\mathbf{i}}
$$

A variable $\Delta t_{i}$ at each cell is governed by

$$
\frac{A_{i}}{\Delta t_{i}}=\frac{\left|u_{i}\right|+c_{i}}{\mathrm{CFL}^{n}}
$$

where $\mathrm{CFL}^{n}=\min \left(1000 ., 1 /\right.$ Residual $\left.^{n}\right)$. Typically the global residual starts at a value of order 1 and decreases as convergence is achieved to $10^{-10}$. Updates to the conserved variable $\Delta \mathbf{q}$ are preprocessed by a safety factor $c_{\text {safe }}$. The value of $c_{\text {safe }}$ has a maximum value of 0.25 but will be made smaller to prevent the change of positive definite quantities $\rho$ or $e$ from exceeding $90 \%$ of their current value. Larger, more aggressive settings for $c_{s a f e}$ will work for some of the smaller area ratio problems but are not documented here.

Figures 4 - 6 present the Mach number distribution and error in Mach number from inflow to shock (isentropic domain) for each nozzle. Solid lines indicate results from Roe's scheme ${ }^{5}$ with a Symmetric Total Variation Diminishing (STVD) ${ }^{11,12}$ limiter. Dashed lines indicate semi-analytic reconstruction using the end-point wave scheme (EPWS). Variations of EPWS with $\phi=1$ (global application cell Reynolds number limiting) and $\phi=\phi(\omega)$ with $\omega=4$ (local application of cell Reynolds number limiting within 4 cells of a critical point) are tested. Results for five levels of grid refinement are documented. The global view of Mach number shows little difference between either of the EPWS formulations and Roe's method. All of the algorithms remain stable from nearly incompressible to hypersonic domains. Significant differences are evident as scale is refined. The exact solution in Figs. 4 - 6 refers to an inviscid system of equations. All of the methods produce larger errors in the throat (transonic domain) than in either the subsonic or supersonic domain - probably associated with the introduction of the limiting algorithm. Error magnitudes increase with increasing maximum area ratio as a result of larger rate of area variation.

Error at $x=-0.5$ (subsonic domain) for the area ratio 2,000 case is plotted as a function of mesh size for the Roe and EPWS schemes in Fig. 7. The slope of the error curve is approximately 2 for Roe and EPWS with $\phi=\phi(\omega)$ indicating second-order accuracy. The slope of the error curve for EPWS with $\phi=1$ is approximately 1 indicating first-order accuracy. These results are representative of those observed for other area ratios and at other positions along the nozzle away from the throat and the shock. The constant $\phi$ formulation adds a dissipation term linearly proportional to the cell Reynolds number up to the point where the cell Reynolds number is less than 8 . For the present test case where the cell Reynolds number exceeds 8 everywhere the added diffusivity works like a first-order dissipation. The Roe's scheme engages a limiter and the EPWS scheme with $\phi=\phi(\omega)$ engages added diffusivity only at isolated locations in the domain so that second-order properties appear to recover away from these isolated locations. The EPWS scheme achieves this order property with a 3 point stencil while the Roe scheme requires a 5 point stencil for the STVD limiter. All subsequent figures for the nozzle problem will use the EPWS scheme with $\phi=\phi(\omega)$.

Figure 8 presents temperature distributions in the nozzle for the baseline stagnation pressure $\left(p_{\text {stag }}=10^{6} \mathrm{~Pa}\right)$ and a lower stagnation pressure $\left(p_{\text {stag }}=10^{3} \mathrm{~Pa}\right)$. The cell Reynolds number 


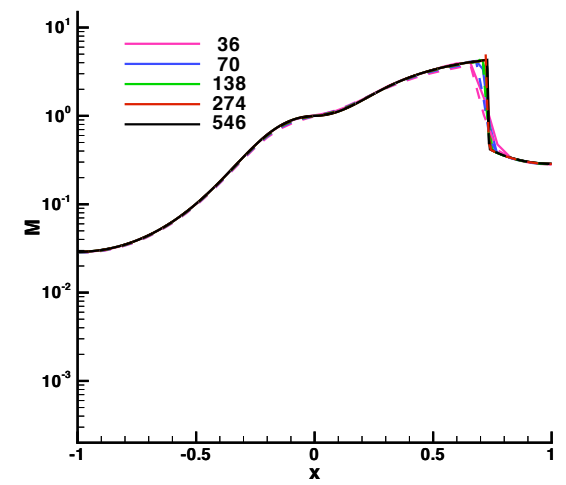

(a) Mach number, Roe - solid, EPWS $\phi=1$ - dashed

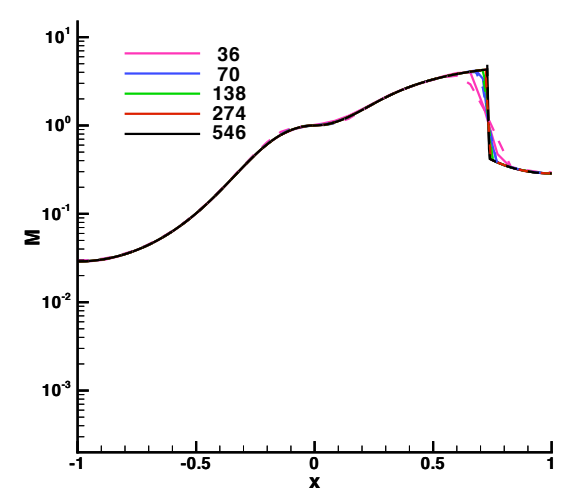

(c) Mach number, Roe - solid, EPWS $\phi=\phi(\omega)$ - dashed

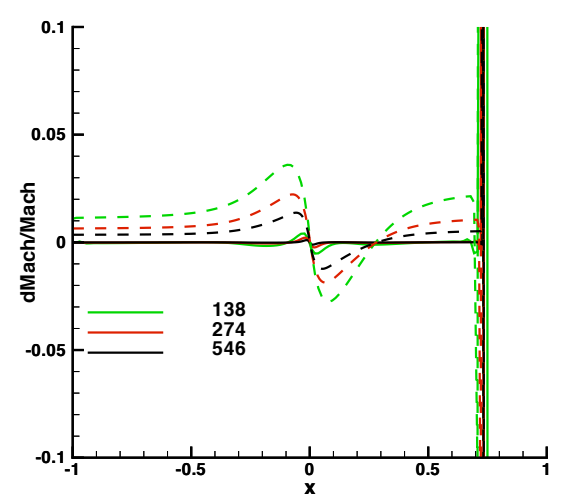

(b) $\left(M-M_{\text {exact }}\right) / M_{\text {exact }}$, Roe - solid, EPWS $\phi=1$ dashed

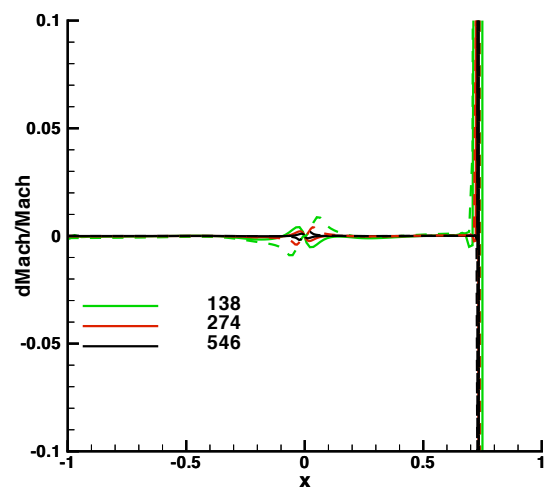

(d) $\left(M-M_{\text {exact }}\right) / M_{\text {exact }}$, Roe - solid, EPWS $\phi=\phi(\omega)$ dashed

Figure 4. Mach number distribution and error for $\frac{A_{\text {exit }}}{A_{\text {throat }}}=20$.

crossing the shock is of order 1000 for the baseline stagnation pressure (Fig. 9(a)) and is of order 1 for the lower stagnation pressure (Fig. 9(b)). Shock structure is resolved for cell Reynolds numbers of order 1 or smaller while an abrupt jump spanning two cells is obtained for the higher cell Reynolds numbers (limited to a value of 8 in Eq. 45 and Eq. 46 with $\phi=1$ ). In both cases, profile convergence is observed with grid refinement. Also, the Roe scheme (solid line) and the semi-analytic EPWS scheme (dashed line) converge with grid refinement.

Details of the temperature distribution at the throat and behind the shock are presented in Figures 10 - 12. Both the EPWS and Roe's scheme show a temperature overshoot approaching the throat for the two largest area ratios with the largest source terms. The overshoot is less pronounced for the EPWS reconstruction which includes the source term in the reconstruction process. Both the Roe and the EPWS schemes present a smooth transition across the shock.

The EPWS scheme requires approximately four times the CPU time per relaxation step for the coupled viscous and inviscid flux reconstruction as compared to the Roe scheme as seen in Table 10. Only results for the maximum area ratio equal to 20 are shown because the limiter used in the 


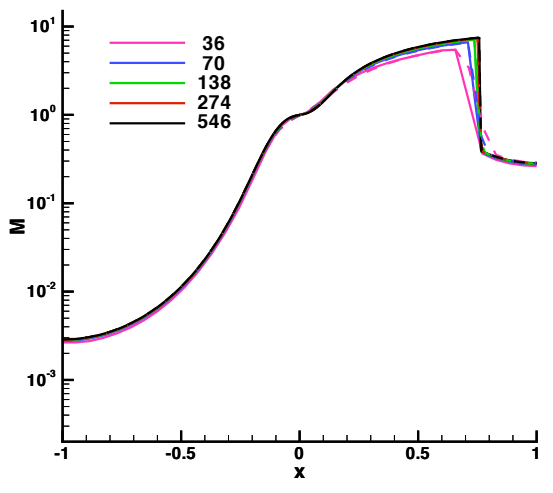

(a) Mach number, Roe - solid, EPWS $\phi=1$ - dashed

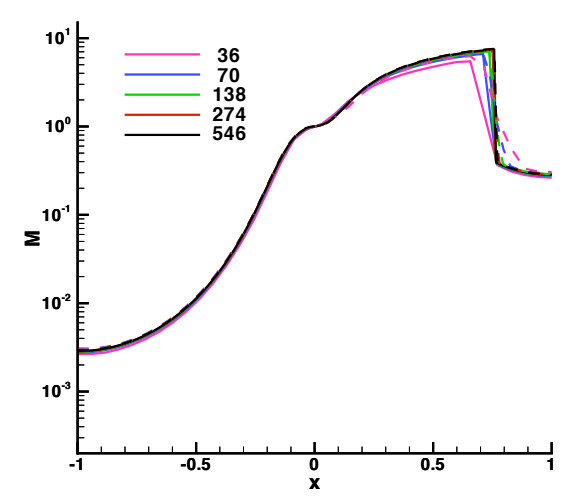

(c) Mach number, Roe - solid, EPWS $\phi=\phi(\omega)$ - dashed

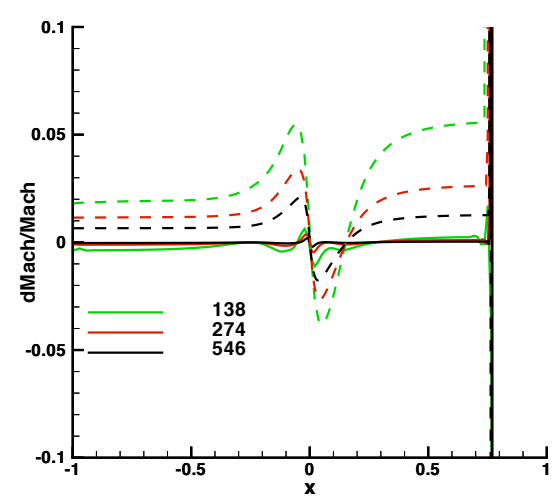

(b) $\left(M-M_{\text {exact }}\right) / M_{\text {exact }}$, Roe - solid, EPWS $\phi=1$ dashed

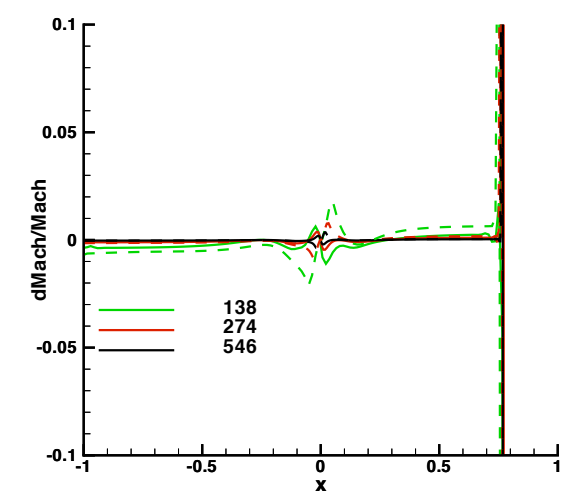

(d) $\left(M-M_{\text {exact }}\right) / M_{\text {exact }}$, Roe - solid, EPWS $\phi=\phi(\omega)$ dashed

Figure 5. Mach number distribution and error for $\frac{A_{\text {exit }}}{A_{\text {throat }}}=200$.

Roe algorithm hung for the larger area ratio cases. The EPWS scheme usually converges in fewer relaxation steps (column 5) so that total time to convergence (column 3) is roughly equivalent - a result somewhat dependent on the magnitude of ringing and time to damp same associated with the limiter. Both the EPWS and Roe reconstruction use identical relaxation algorithm. The coarsest grid (36 cells) is started from a crude initialization; subsequent grids are initialized by interpolation from the previous converged solution. 


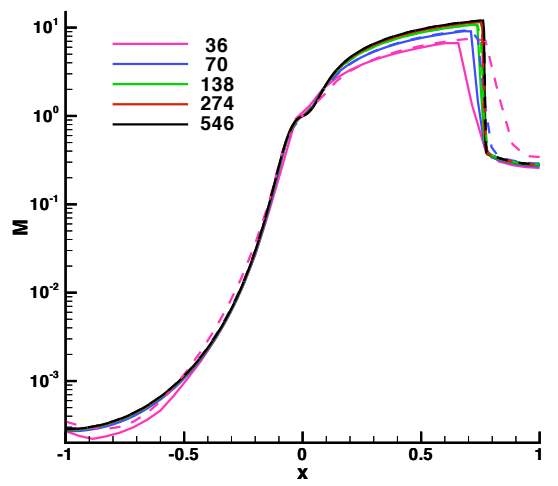

(a) Mach number, Roe - solid, EPWS $\phi=1$ - dashed

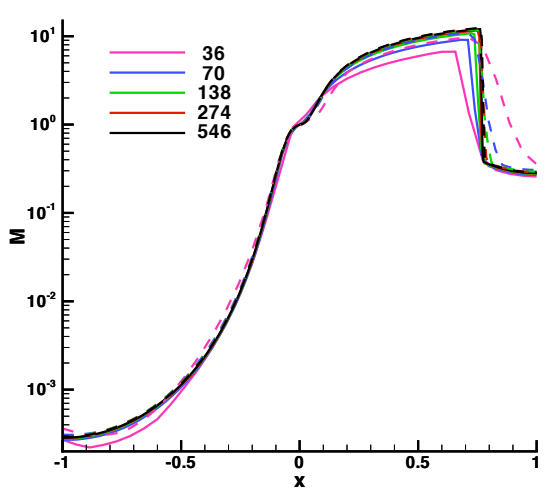

(c) Mach number, Roe - solid, EPWS $\phi=\phi(\omega)$ - dashed

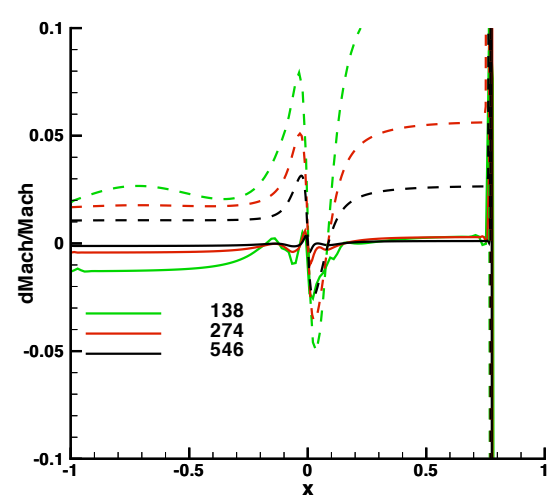

(b) $\left(M-M_{\text {exact }}\right) / M_{\text {exact }}$, Roe - solid, EPWS $\phi=1$ dashed

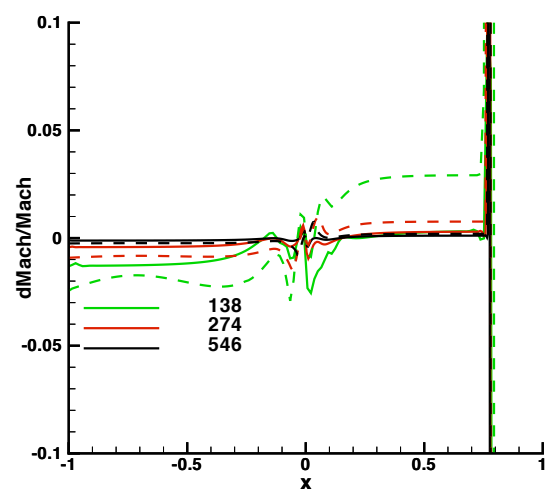

(d) $\left(M-M_{\text {exact }}\right) / M_{\text {exact }}$, Roe - solid, EPWS $\phi=\phi(\omega)$ dashed

Figure 6. Mach number distribution and error for $\frac{A_{\text {exit }}}{A_{\text {throat }}}=2,000$. 


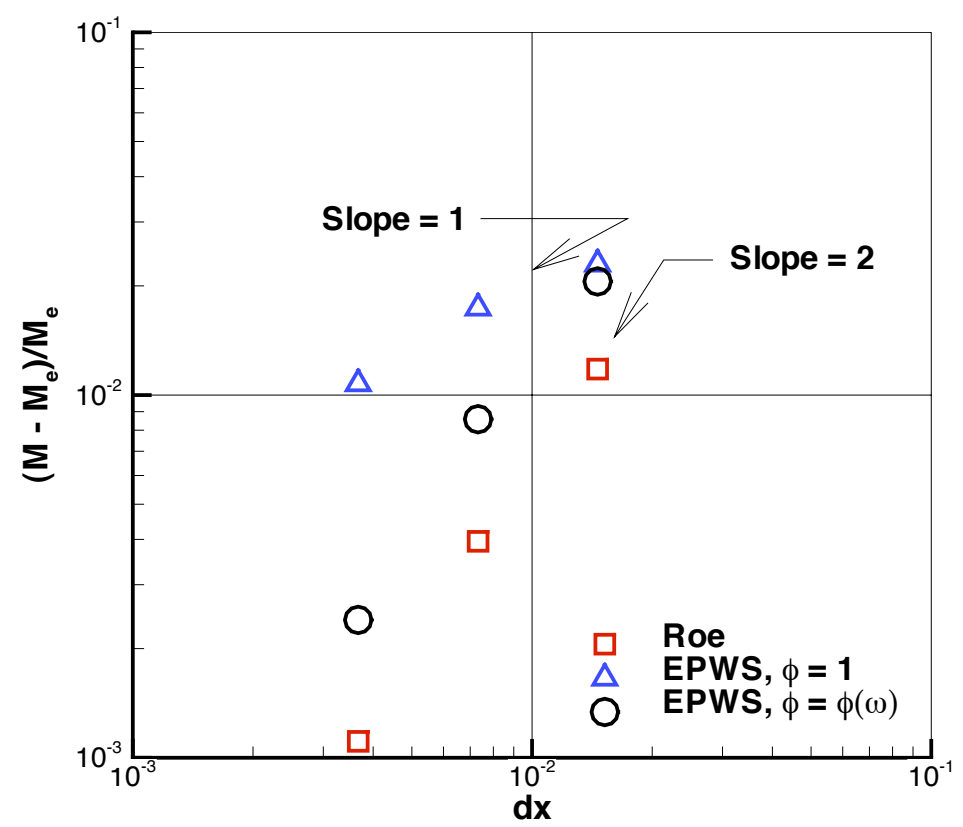

Figure 7. Grid convergence at $x=-0.5$ for the area ratio 2000 case indicating order properties of the EPWS and Roe formulations.

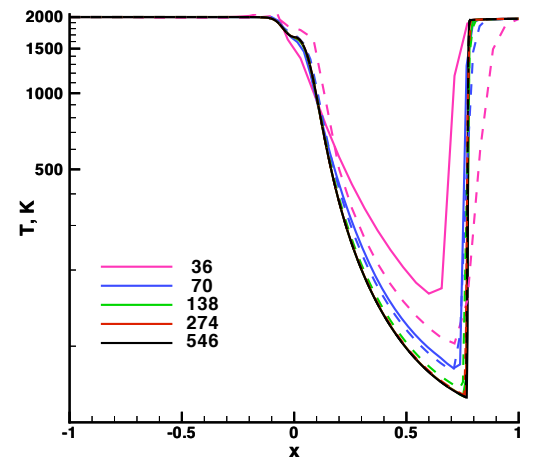

(a) $p_{\text {stag }}=10^{6} \mathrm{~Pa}$, Roe - solid, EPWS $\phi=\phi(\omega)-$ dashed

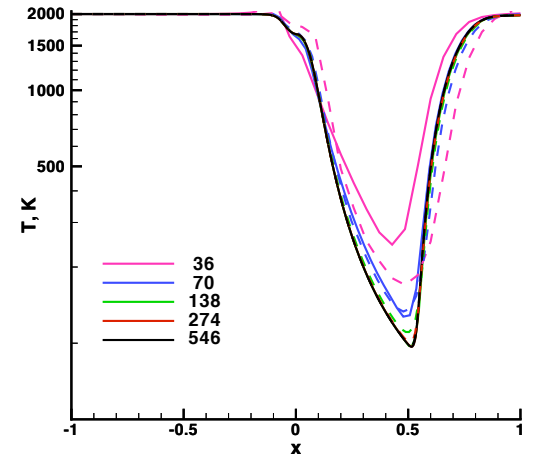

(b) $p_{\text {stag }}=10^{3} \mathrm{~Pa}$, Roe - solid, EPWS $\phi=\phi(\omega)-$ dashed

Figure 8. Temperature distribution for $\frac{A_{\text {exit }}}{A_{\text {throat }}}=2,000$. 


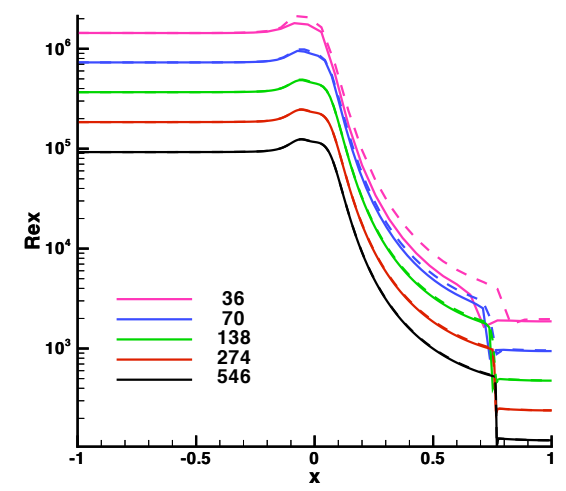

(a) $p_{\text {stag }}=10^{6} \mathrm{~Pa}$, Roe - solid, EPWS - dashed

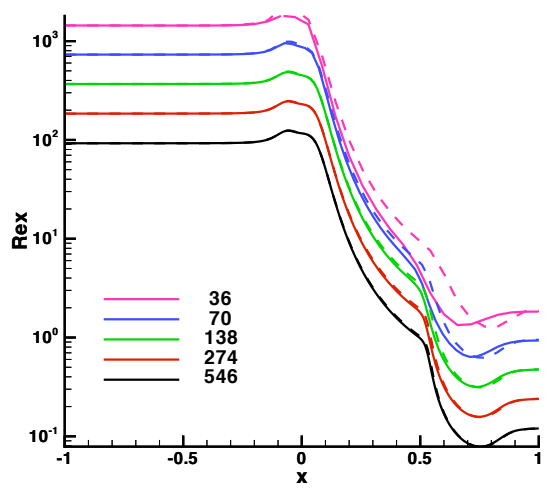

(b) $p_{\text {stag }}=10^{3} \mathrm{~Pa}$, Roe - solid, EPWS - dashed

Figure 9. Cell Reynolds number distribution for $\frac{A_{\text {exit }}}{A_{\text {throat }}}=2,000$.

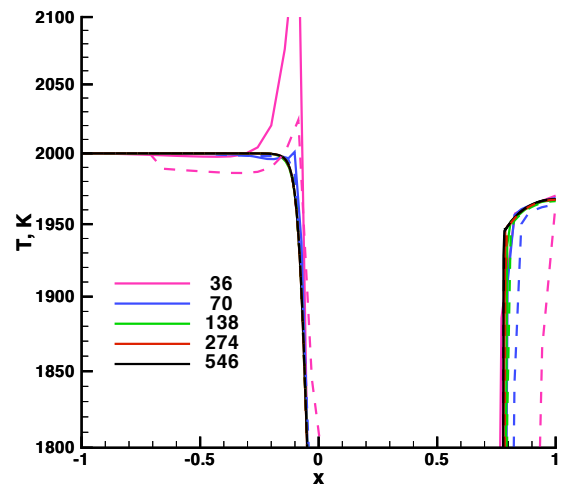

(a) Throat, Roe vs EPWS $\phi=\phi(\omega)$

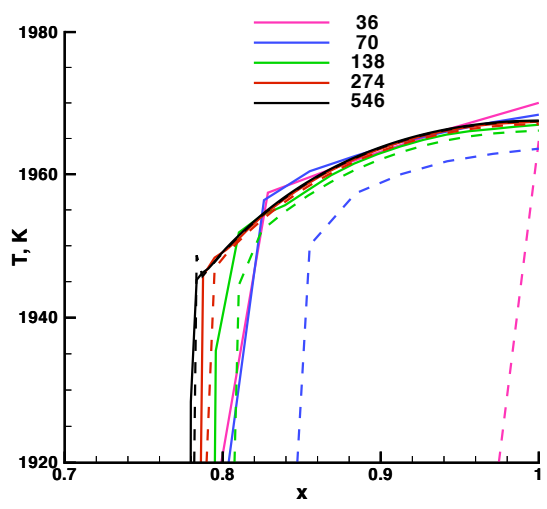

(b) Shock, Roe vs EPWS $\phi=\phi(\omega)$

Figure 10. Temperature distribution for $\frac{A_{\text {exit }}}{A_{\text {throat }}}=2,000$. 


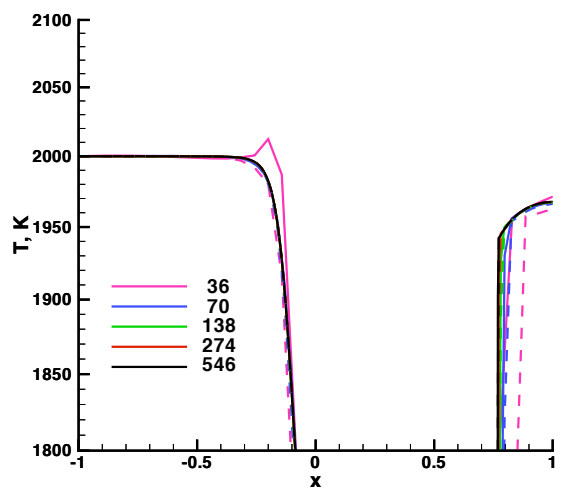

(a) Throat, Roe vs EPWS $\phi=\phi(\omega)$

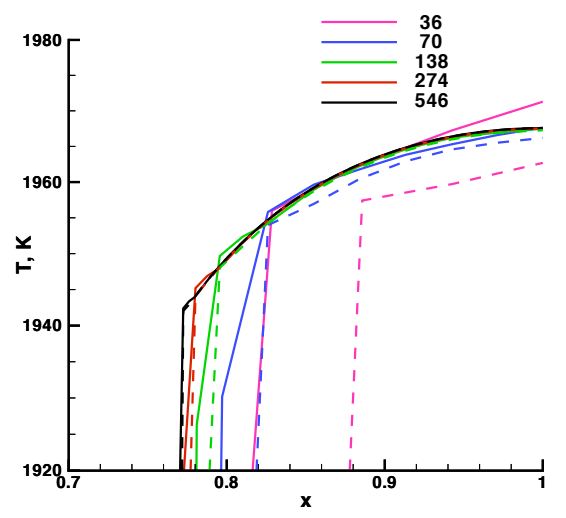

(b) Shock, Roe vs EPWS $\phi=\phi(\omega)$

Figure 11. Temperature distribution for $\frac{A_{\text {exit }}}{A_{\text {throat }}}=200$.

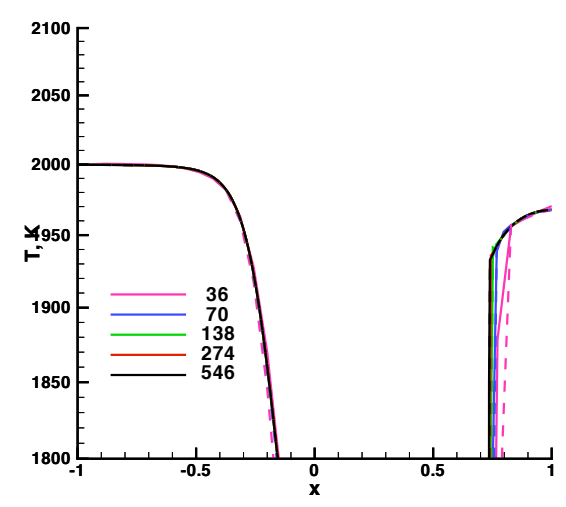

(a) Throat, Roe vs EPWS $\phi=1$ with $\phi=\phi(\omega)$

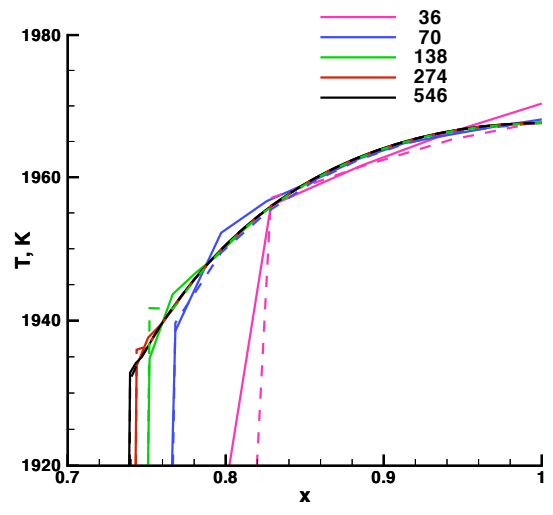

(b) Shock, Roe vs EPWS $\phi=\phi(\omega)$

Figure 12. Temperature distribution for $\frac{A_{\text {exit }}}{A_{\text {throat }}}=20$. 


\section{Multi-Dimensional Vector Equation}

Equations are written in a locally convenient, Cartesian coordinate system $(X, Y, Z)$. In the applications described herein $X$ is aligned with a line segment $\vec{x}_{R}-\vec{x}_{L}$ with origin at the midpoint of the line segment. In future work, it may be advantageous to align $X$ with convective velocity $\vec{u}$ with origin at the centroid of a tetrahedron. In either case, the transformation from reference system $\vec{x}$ to local system $\vec{X}$ and back is given by:

$$
\begin{aligned}
{\left[\begin{array}{l}
d X \\
d Y \\
d Z
\end{array}\right] } & =\left[\begin{array}{ccc}
n_{x} & n_{y} & n_{z} \\
l_{x} & l_{y} & l_{z} \\
m_{x} & m_{y} & m_{z}
\end{array}\right]\left[\begin{array}{l}
d x \\
d y \\
d z
\end{array}\right] \\
{\left[\begin{array}{l}
d x \\
d y \\
d z
\end{array}\right] } & =\left[\begin{array}{lll}
n_{x} & l_{x} & m_{x} \\
n_{y} & l_{y} & m_{y} \\
n_{z} & l_{z} & m_{z}
\end{array}\right]\left[\begin{array}{l}
d X \\
d Y \\
d Z
\end{array}\right]
\end{aligned}
$$

Velocities are transformed in like manner.

$$
\begin{aligned}
{\left[\begin{array}{c}
U \\
V \\
W
\end{array}\right] } & =\left[\begin{array}{ccc}
n_{x} & n_{y} & n_{z} \\
l_{x} & l_{y} & l_{z} \\
m_{x} & m_{y} & m_{z}
\end{array}\right]\left[\begin{array}{c}
u \\
v \\
w
\end{array}\right] \\
{\left[\begin{array}{c}
u \\
v \\
w
\end{array}\right] } & =\left[\begin{array}{ccc}
n_{x} & l_{x} & m_{x} \\
n_{y} & l_{y} & m_{y} \\
n_{z} & l_{z} & m_{z}
\end{array}\right]\left[\begin{array}{c}
U \\
V \\
W
\end{array}\right]
\end{aligned}
$$

In the case of $X$ aligned with the line segment the transformation is defined with $n_{x}=d x / d s$, $n_{y}=d y / d s$, and $n_{z}=d z / d s$ where $d s=\sqrt{d x^{2}+d y^{2}+d z^{2}}$. Unit vectors $\vec{l}$ and $\vec{m}$ are mutually orthogonal.

The conservation equations include an inviscid part $\mathbf{f}$, a viscous part $\mathbf{h}$ and a source term $\mathbf{s}$ that govern the evolution of conserved variable $\mathbf{q}$.

$$
\frac{\partial \mathbf{q}}{\partial t}+\frac{\partial\left(\mathbf{f}_{\mathbf{1}}-\mathbf{h}_{\mathbf{1}}\right)}{\partial X}+\frac{\partial\left(\mathbf{f}_{\mathbf{2}}-\mathbf{h}_{\mathbf{2}}\right)}{\partial Y}+\frac{\partial\left(\mathbf{f}_{\mathbf{3}}-\mathbf{h}_{\mathbf{3}}\right)}{\partial Z}=\mathbf{s}
$$

A solution to a steady, linearized approximation to Eq. 106 is sought of the form $\mathbf{q}(X, Y, Z)=$ $\mathbf{q}_{0}+\mathbf{q}_{1}(X)+\mathbf{q}_{2}(Y)+\mathbf{q}_{3}(Z)$. The viscous terms defining $\mathbf{h}$ involve derivatives of $\tilde{\mathbf{q}}$ with respect to $X$, $Y$, and $Z(\mathbf{h}[\tilde{\mathbf{q}}, \partial \tilde{\mathbf{q}} / \partial X, \partial \tilde{\mathbf{q}} / \partial Y, \partial \tilde{\mathbf{q}} / \partial Z])$. Because of the separation of variables in the assumed form for $\mathbf{q}$ (and implicitly $\tilde{\mathbf{q}}$ ) the only parts of $\mathbf{h}_{1}$ to survive the linearization involve second derivatives of $\tilde{\mathbf{q}}$ with respect to $X$. In like manner, the linearization of $\mathbf{h}_{2}$ include only second derivatives with respect to $Y$ and the linearization of $\mathbf{h}_{3}$ include only second derivatives with respect to $Z$. All cross derivative terms are annihilated. The steady, linearized form of Eq. 106 thus becomes:

$$
\mathbf{A}_{1} \frac{\partial \mathbf{q}_{1}}{\partial X}-\nu \tilde{\mathbf{B}}_{11} \frac{\partial^{2} \tilde{\mathbf{q}}_{1}}{\partial X^{2}}+\mathbf{A}_{2} \frac{\partial \mathbf{q}_{2}}{\partial Y}-\nu \tilde{\mathbf{B}}_{22} \frac{\partial^{2} \tilde{\mathbf{q}}_{2}}{\partial Y^{2}}+\mathbf{A}_{3} \frac{\partial \mathbf{q}_{3}}{\partial X}-\nu \tilde{\mathbf{B}}_{33} \frac{\partial^{2} \tilde{\mathbf{q}}_{3}}{\partial Z^{2}}=\mathbf{s}_{0}
$$

where

$$
\begin{aligned}
\mathbf{A}_{i} & =\frac{\partial \mathbf{f}_{i}}{\partial \mathbf{q}} \\
\nu \tilde{\mathbf{B}}_{i j} & =\frac{\partial \mathbf{h}_{i}}{\partial \frac{\partial \tilde{\mathbf{q}}}{\partial X_{j}}}
\end{aligned}
$$


Isolating the scalar factor $\nu=\mu / \rho$ keeps elements of $\mathbf{B}_{i i}$ of order 1 .

The semi-analytic solution for flux reconstruction must satisfy appropriate boundary conditions in addition to Eq. 107. However, boundary values are only defined at nodes. Consequently, there is an infinite degree of freedom in how one may construct a solution that needs to satisfy boundary conditions at isolated nodes. It is this freedom that accommodates a solution employing separation of variables. This same freedom introduces ambiguity in how one handles the effect of source term $\mathbf{s}_{0}$ on the solution of $\mathbf{q}_{1}, \mathbf{q}_{2}$, and $\mathbf{q}_{3}$. The approach taken here is to distribute the effect of $\mathbf{s}_{0}$ evenly over the contributions from the three coordinate directions. Thus, solutions are derived for separable pieces of Eq. 107.

$$
\begin{aligned}
\mathbf{A}_{1} \frac{\partial \mathbf{q}_{1}}{\partial X} & =\nu \tilde{\mathbf{B}}_{11} \frac{\partial^{2} \tilde{\mathbf{q}}_{1}}{\partial X^{2}}+\frac{1}{3} \mathbf{s}_{0} \\
\mathbf{A}_{2} \frac{\partial \mathbf{q}_{2}}{\partial Y} & =\nu \tilde{\mathbf{B}}_{22} \frac{\partial^{2} \tilde{\mathbf{q}}_{2}}{\partial Y^{2}}+\frac{1}{3} \mathbf{s}_{0} \\
\mathbf{A}_{3} \frac{\partial \mathbf{q}_{3}}{\partial X} & =\nu \tilde{\mathbf{B}}_{33} \frac{\partial^{2} \tilde{\mathbf{q}}_{3}}{\partial Z^{2}}+\frac{1}{3} \mathbf{s}_{0}
\end{aligned}
$$

Solutions to Eqs. 110 - 112 were defined in the previous section in Eqs. 66 - 67 with $\mathbf{R}_{i} \mathbf{\Psi}_{i}\left(X_{i}\right)$ and $\mathbf{\Phi}_{i}\left(X_{i}\right)$ defined in Eqs. 62 and 63. The matrices for three-dimensional flow of a perfect gas and for a mixture of thermally perfect gases in thermochemical nonequilibrium are defined in Appendix B.

$$
\begin{aligned}
& \mathbf{q}_{1}=\mathbf{q}_{1,0}+\mathbf{R}_{1} \boldsymbol{\Psi}_{1}(X) \mathbf{R}_{1}^{-1}\left(\frac{d \mathbf{q}}{d X}\right)_{1,0}+\mathbf{R}_{1} \boldsymbol{\Phi}_{1}(X) \mathbf{R}_{1}^{-1} \frac{1}{3} \mathbf{s}_{0} \\
& \mathbf{q}_{2}=\mathbf{q}_{2,0}+\mathbf{R}_{2} \boldsymbol{\Psi}_{2}(Y) \mathbf{R}_{2}^{-1}\left(\frac{d \mathbf{q}}{d Y}\right)_{2,0}+\mathbf{R}_{2} \boldsymbol{\Phi}_{2}(Y) \mathbf{R}_{2}^{-1} \frac{1}{3} \mathbf{s}_{0} \\
& \mathbf{q}_{3}=\mathbf{q}_{3,0}+\mathbf{R}_{3} \boldsymbol{\Psi}_{3}(Z) \mathbf{R}_{3}^{-1}\left(\frac{d \mathbf{q}}{d Z}\right)_{3,0}+\mathbf{R}_{3} \boldsymbol{\Phi}_{3}(Z) \mathbf{R}_{3}^{-1} \frac{1}{3} \mathbf{s}_{0}
\end{aligned}
$$

The solution for $\mathbf{q}$ is obtained by summing Eqs. 113 - 115 .

$$
\begin{aligned}
\mathbf{q} & =\mathbf{q}_{0}+\mathbf{R}_{1} \boldsymbol{\Psi}_{1}(X) \mathbf{R}_{1}^{-1}\left(\frac{\partial \mathbf{q}}{\partial X}\right)_{0}+\mathbf{R}_{2} \boldsymbol{\Psi}_{2}(Y) \mathbf{R}_{2}^{-1}\left(\frac{\partial \mathbf{q}}{\partial Y}\right)_{0}+\mathbf{R}_{3} \boldsymbol{\Psi}_{3}(Z) \mathbf{R}_{3}^{-1}\left(\frac{\partial \mathbf{q}}{\partial Z}\right)_{0} \\
& +\frac{1}{3}\left(\mathbf{R}_{1} \boldsymbol{\Phi}_{1}(X) \mathbf{R}_{1}^{-1}+\mathbf{R}_{2} \boldsymbol{\Phi}_{2}(Y) \mathbf{R}_{2}^{-1}+\mathbf{R}_{3} \boldsymbol{\Phi}_{3}(Z) \mathbf{R}_{3}^{-1}\right) \mathbf{s}_{0}
\end{aligned}
$$

Equation 116 requires evaluation of four vectors: $\mathbf{q}_{0},\left(\frac{\partial \mathbf{q}}{\partial X}\right)_{0},\left(\frac{\partial \mathbf{q}}{\partial Y}\right)_{0}$, and $\left(\frac{\partial \mathbf{q}}{\partial Z}\right)_{0}$. These vectors can be evaluated implicitly by applying known boundary values $\mathbf{q}_{A}, \mathbf{q}_{B}, \mathbf{q}_{C}$, and $\mathbf{q}_{D}$ at nodes $A$, $B, C$, and $D$.

$$
\begin{aligned}
\mathbf{q}_{A} & =\mathbf{q}_{0}+\mathbf{R}_{1} \boldsymbol{\Psi}_{1, A} \mathbf{R}_{1}^{-1}\left(\frac{\partial \mathbf{q}}{\partial X}\right)_{0}+\mathbf{R}_{2} \boldsymbol{\Psi}_{2, A} \mathbf{R}_{2}^{-1}\left(\frac{\partial \mathbf{q}}{\partial Y}\right)_{0}+\mathbf{R}_{3} \boldsymbol{\Psi}_{3, A} \mathbf{R}_{3}^{-1}\left(\frac{\partial \mathbf{q}}{\partial Z}\right)_{0} \\
& +\frac{1}{3}\left(\mathbf{R}_{1} \boldsymbol{\Phi}_{1, A} \mathbf{R}_{1}^{-1}+\mathbf{R}_{2} \boldsymbol{\Phi}_{2, A} \mathbf{R}_{2}^{-1}+\mathbf{R}_{3} \boldsymbol{\Phi}_{3, A} \mathbf{R}_{3}^{-1}\right) \mathbf{s}_{0} \\
\mathbf{q}_{B} & =\mathbf{q}_{0}+\mathbf{R}_{1} \boldsymbol{\Psi}_{1, B} \mathbf{R}_{1}^{-1}\left(\frac{\partial \mathbf{q}}{\partial X}\right)_{0}+\mathbf{R}_{2} \boldsymbol{\Psi}_{2, B} \mathbf{R}_{2}^{-1}\left(\frac{\partial \mathbf{q}}{\partial Y}\right)_{0}+\mathbf{R}_{3} \boldsymbol{\Psi}_{3, B} \mathbf{R}_{3}^{-1}\left(\frac{\partial \mathbf{q}}{\partial Z}\right)_{0} \\
& +\frac{1}{3}\left(\mathbf{R}_{1} \boldsymbol{\Phi}_{1, B} \mathbf{R}_{1}^{-1}+\mathbf{R}_{2} \boldsymbol{\Phi}_{2, B} \mathbf{R}_{2}^{-1}+\mathbf{R}_{3} \boldsymbol{\Phi}_{3, B} \mathbf{R}_{3}^{-1}\right)_{0} \\
\mathbf{q}_{C} & =\mathbf{q}_{0}+\mathbf{R}_{1} \boldsymbol{\Psi}_{1, C} \mathbf{R}_{1}^{-1}\left(\frac{\partial \mathbf{q}}{\partial X}\right)_{0}+\mathbf{R}_{2} \boldsymbol{\Psi}_{2, C} \mathbf{R}_{2}^{-1}\left(\frac{\partial \mathbf{q}}{\partial Y}\right)_{0}+\mathbf{R}_{3} \boldsymbol{\Psi}_{3, C} \mathbf{R}_{3}^{-1}\left(\frac{\partial \mathbf{q}}{\partial Z}\right)_{0} \\
& +\frac{1}{3}\left(\mathbf{R}_{1} \boldsymbol{\Phi}_{1, C} \mathbf{R}_{1}^{-1}+\mathbf{R}_{2} \boldsymbol{\Phi}_{2, C} \mathbf{R}_{2}^{-1}+\mathbf{R}_{3} \boldsymbol{\Phi}_{3, C} \mathbf{R}_{3}^{-1}\right) \mathbf{s}_{0} \\
\mathbf{q}_{D} & =\mathbf{q}_{0}+\mathbf{R}_{1} \mathbf{\Psi}_{1, D} \mathbf{R}_{1}^{-1}\left(\frac{\partial \mathbf{q}}{\partial X}\right)_{0}+\mathbf{R}_{2} \mathbf{\Psi}_{2, D} \mathbf{R}_{2}^{-1}\left(\frac{\partial \mathbf{q}}{\partial Y}\right)_{0}+\mathbf{R}_{3} \boldsymbol{\Psi}_{3, D} \mathbf{R}_{3}^{-1}\left(\frac{\partial \mathbf{q}}{\partial Z}\right)_{0} \\
& +\frac{1}{3}\left(\mathbf{R}_{1} \boldsymbol{\Phi}_{1, D} \mathbf{R}_{1}^{-1}+\mathbf{R}_{2} \boldsymbol{\Phi}_{2, D} \mathbf{R}_{2}^{-1}+\mathbf{R}_{3} \boldsymbol{\Phi}_{3, D} \mathbf{R}_{3}^{-1}\right) \mathbf{s}_{0}
\end{aligned}
$$


Equation 117 can be simplified as shown below in Eq. 118, however, it still requires an implicit solution of a $15 \times 15$ matrix for even a perfect gas. Additional simplifications are possible to eliminate one of the remaining gradient vectors resulting in a $10 \times 10$ matrix for a perfect gas but ultimately an implicit solution is required, even for the EPWS algorithm. (In the EPWS formulation, one would substitute each of the nodal subscripts $A, B, C, D$ in turn for the centroid subscript 0.) Consequently, the formulation of a fully three-dimensional reconstruction requires significantly more operations to handle the implicit solution than the one-dimensional systems which are handled explicitly.

$$
\begin{aligned}
& \mathbf{q}_{A}-\mathbf{q}_{B}=\mathbf{R}_{1}\left(\boldsymbol{\Psi}_{1, A}-\boldsymbol{\Psi}_{1, B}\right) \mathbf{R}_{1}^{-1}\left(\frac{\partial \mathbf{q}}{\partial X}\right)_{0}+\mathbf{R}_{2}\left(\boldsymbol{\Psi}_{2, A}-\boldsymbol{\Psi}_{2, B}\right) \mathbf{R}_{2}^{-1}\left(\frac{\partial \mathbf{q}}{\partial Y}\right)_{0} \\
& +\mathbf{R}_{3}\left(\boldsymbol{\Psi}_{3, A}-\boldsymbol{\Psi}_{3, B}\right) \mathbf{R}_{3}^{-1}\left(\frac{\partial \mathbf{q}}{\partial Z}\right)_{0} \\
& +\frac{1}{3}\left[\mathbf{R}_{1}\left(\boldsymbol{\Phi}_{1, A}-\boldsymbol{\Phi}_{1, B}\right) \mathbf{R}_{1}^{-1}+\mathbf{R}_{2}\left(\boldsymbol{\Phi}_{2, A}-\boldsymbol{\Phi}_{2, B}\right) \mathbf{R}_{2}^{-1}+\mathbf{R}_{3}\left(\boldsymbol{\Phi}_{3, A}-\boldsymbol{\Phi}_{3, B}\right) \mathbf{R}_{3}^{-1}\right] \mathbf{s}_{0} \\
& \mathbf{q}_{B}-\mathbf{q}_{C}=\mathbf{R}_{1}\left(\boldsymbol{\Psi}_{1, B}-\boldsymbol{\Psi}_{1, C}\right) \mathbf{R}_{1}^{-1}\left(\frac{\partial \mathbf{q}}{\partial X}\right)_{0}+\mathbf{R}_{2}\left(\boldsymbol{\Psi}_{2, B}-\boldsymbol{\Psi}_{2, C}\right) \mathbf{R}_{2}^{-1}\left(\frac{\partial \mathbf{q}}{\partial Y}\right)_{0} \\
& +\mathbf{R}_{3}\left(\boldsymbol{\Psi}_{3, B}-\boldsymbol{\Psi}_{3, C}\right) \mathbf{R}_{3}^{-1}\left(\frac{\partial \mathbf{q}}{\partial Z}\right)_{0} \\
& +\frac{1}{3}\left[\mathbf{R}_{1}\left(\boldsymbol{\Phi}_{1, B}-\boldsymbol{\Phi}_{1, C}\right) \mathbf{R}_{1}^{-1}+\mathbf{R}_{2}\left(\boldsymbol{\Phi}_{2, B}-\boldsymbol{\Phi}_{2, C}\right) \mathbf{R}_{2}^{-1}+\mathbf{R}_{3}\left(\boldsymbol{\Phi}_{3, B}-\boldsymbol{\Phi}_{3, C}\right) \mathbf{R}_{3}^{-1}\right] \mathbf{s}_{0} \\
& \mathbf{q}_{C}-\mathbf{q}_{D}=\mathbf{R}_{1}\left(\boldsymbol{\Psi}_{1, C}-\boldsymbol{\Psi}_{1, D}\right) \mathbf{R}_{1}^{-1}\left(\frac{\partial \mathbf{q}}{\partial X}\right)_{0}+\mathbf{R}_{2}\left(\boldsymbol{\Psi}_{2, C}-\boldsymbol{\Psi}_{2, D}\right) \mathbf{R}_{2}^{-1}\left(\frac{\partial \mathbf{q}}{\partial Y}\right)_{0} \\
& +\mathbf{R}_{3}\left(\boldsymbol{\Psi}_{3, C}-\boldsymbol{\Psi}_{3, D}\right) \mathbf{R}_{3}^{-1}\left(\frac{\partial \mathbf{q}}{\partial Z}\right)_{0} \\
& +\frac{1}{3}\left[\mathbf{R}_{1}\left(\boldsymbol{\Phi}_{1, C}-\boldsymbol{\Phi}_{1, D}\right) \mathbf{R}_{1}^{-1}+\mathbf{R}_{2}\left(\boldsymbol{\Phi}_{2, C}-\boldsymbol{\Phi}_{2, D}\right) \mathbf{R}_{2}^{-1}+\mathbf{R}_{3}\left(\boldsymbol{\Phi}_{3, C}-\boldsymbol{\Phi}_{3, D}\right) \mathbf{R}_{3}^{-1}\right] \mathbf{s}_{0} \\
& d \mathbf{q}_{A-B}=\mathbf{R}_{1} d \boldsymbol{\Psi}_{1, A-B} \mathbf{R}_{1}^{-1}\left(\frac{\partial \mathbf{q}}{\partial X}\right)_{0}+\mathbf{R}_{2} d \boldsymbol{\Psi}_{2, A-B} \mathbf{R}_{2}^{-1}\left(\frac{\partial \mathbf{q}}{\partial Y}\right)_{0} \\
& +\mathbf{R}_{3} d \boldsymbol{\Psi}_{3, A-B} \mathbf{R}_{3}^{-1}\left(\frac{\partial \mathbf{q}}{\partial Z}\right)_{0} \\
& +\frac{1}{3}\left[\mathbf{R}_{1} d \boldsymbol{\Phi}_{1, A-B} \mathbf{R}_{1}^{-1}+\mathbf{R}_{2} d \boldsymbol{\Phi}_{2, A-B} \mathbf{R}_{2}^{-1}+\mathbf{R}_{3} d \boldsymbol{\Phi}_{3, A-B} \mathbf{R}_{3}^{-1}\right] \mathbf{s}_{0} \\
& d \mathbf{q}_{B-C}=\mathbf{R}_{1} d \boldsymbol{\Psi}_{1, B-C} \mathbf{R}_{1}^{-1}\left(\frac{\partial \mathbf{q}}{\partial X}\right)_{0}+\mathbf{R}_{2} d \boldsymbol{\Psi}_{2, B-C} \mathbf{R}_{2}^{-1}\left(\frac{\partial \mathbf{q}}{\partial Y}\right)_{0} \\
& +\mathbf{R}_{3} d \mathbf{\Psi}_{3, B-C} \mathbf{R}_{3}^{-1}\left(\frac{\partial \mathbf{q}}{\partial Z}\right)_{0} \\
& +\frac{1}{3}\left[\mathbf{R}_{1} d \boldsymbol{\Phi}_{1, B-C} \mathbf{R}_{1}^{-1}+\mathbf{R}_{2} d \boldsymbol{\Phi}_{2, B-C} \mathbf{R}_{2}^{-1}+\mathbf{R}_{3} d \boldsymbol{\Phi}_{3, B-C} \mathbf{R}_{3}^{-1}\right] \mathbf{s}_{0} \\
& d \mathbf{q}_{C-D}=\mathbf{R}_{1} d \boldsymbol{\Psi}_{1, C-D} \mathbf{R}_{1}^{-1}\left(\frac{\partial \mathbf{q}}{\partial X}\right)_{0}+\mathbf{R}_{2} d \boldsymbol{\Psi}_{2, C-D} \mathbf{R}_{2}^{-1}\left(\frac{\partial \mathbf{q}}{\partial Y}\right)_{0} \\
& +\mathbf{R}_{3}\left(d \boldsymbol{\Psi}_{3, C-D} \mathbf{R}_{3}^{-1}\left(\frac{\partial \mathbf{q}}{\partial Z}\right)_{0}\right. \\
& +\frac{1}{3}\left[\mathbf{R}_{1} d \boldsymbol{\Phi}_{1, C-D} \mathbf{R}_{1}^{-1}+\mathbf{R}_{2} d \boldsymbol{\Phi}_{2, C-D} \mathbf{R}_{2}^{-1}+\mathbf{R}_{3} d \boldsymbol{\Phi}_{3, C-D} \mathbf{R}_{3}^{-1}\right] \mathbf{s}_{0}
\end{aligned}
$$

An explicit formulation can be recovered if an edge specific, one-dimensional (as opposed to volume specific, three-dimensional) reconstruction is accepted. In this case, align $X$ between the nodes $A$ and $B$. Independent variables $Y$ and $Z$ are identically equal to zero on this edge. Consequently, $\mathbf{\Psi}_{2}(Y), \boldsymbol{\Psi}_{3}(Z), \mathbf{\Phi}_{2}(Y)$, and $\boldsymbol{\Phi}_{3}(Z)$ are identically zero on this edge. Boundary conditions at nodes $A$ and $B$ are sufficient for defining the semi-analytic flux at any point on this line segment. The reconstruction algorithm now plays out exactly as described in the previous section for one-dimensional systems with appropriate substitution of the matrices for three-dimensional gas flow as defined in Appendix B. Preliminary tests of this simplification (which in fact sacrifices the original intent of multidimensional reconstruction) exhibited stability problems thought to be associated with inadequately moderating the diffusivity in the vicinity of edges where eigenvalues change sign.

Programming the implicit formulation at the centroid of a tetrahedron involves significant changes to the basic structure of existing codes (FUN3D ${ }^{13}$ in this case). The loop over edges 
to create inviscid flux disappears. Computation of the gradient field used for second-order corrections can also be omitted. Flux reconstruction occurs in a loop over centroids in which inviscid and viscous flux contributions are computed simultaneously. The complexity of the reconstruction algorithm should not be trivialized here. If the number of relaxation steps required to evaluate the semi-analytic solution at the centroid is greater than the ratio of total edges to total centroids then the work for semi-analytic reconstruction in 3D carries a penalty (as already documented in the quasi-1D nozzle tests). Application of a directionally dependent diffusivity across critical points must also be derived and tested. The 1D nozzle solutions demonstrate that the approach can be used to capture strong discontinuities with good retention of accuracy. The flexibility to provide truly three-dimensional reconstruction as well as the unified formulation of viscous and inviscid flux provides motivation for continued development.

\section{Concluding Remarks}

Semi-analytic reconstruction uses the analytic solution to a second-order, steady, ordinary differential equation (ODE) to simultaneously evaluate the convective and diffusive flux at all interfaces of a finite volume formulation. Influence of source terms on the reconstructed flux is explicitly included in the formulation. The second-order ODE is itself a linearized approximation to the governing first- and second-order partial differential equation (PDE) conservation laws. Thus, semi-analytic reconstruction defines a family of formulations for finite volume interface fluxes using analytic solutions to approximating equations.

Limiters are not applied in a conventional sense; rather, diffusivity is adjusted in the vicinity of changes in sign of eigenvalues in order to achieve a sufficiently small cell Reynolds number in the analytic formulation across critical points. Cell Reynolds numbers of order 1 are required to analytically span a captured shock without introduction of significant oscillations. This target cell Reynolds number is defined by numerical experiment in the quasi-1D nozzle test problems but the result may be derived analytically for Burgers equation. The target cell Reynolds number is achieved by raising the diffusivity only in the neighborhood of critical points but conceivably grid adaptation could be used to decrease cell size as well.

The reconstruction stencil for the new algorithm is compact, much like a finite element approach. The current approach, however, requires a second-order PDE; it cannot be applied directly to the Euler equations. The approach is finite-volume-based in the sense that conservative flux formulation is the primary goal and reconstructed fluxes are $\mathrm{C} 0$ continuous across cell walls.

Several approaches for application of semi-analytic reconstruction for the solution of onedimensional scalar equations are introduced. Results are compared with exact analytic solutions to Burger's Equation as well as a conventional, upwind discretizations using Roe's scheme. One approach, the end-point wave speed (EPWS) approximation, is further developed for more complex applications. One-dimensional vector equations are tested on a quasi one-dimensional nozzle application. The EPWS algorithm has a more compact difference stencil than Roe's algorithm but reconstruction time is approximately a factor of four larger than for Roe. Though both are second-order accurate schemes, Roe's method approaches a grid converged solution with fewer grid points. Work is ongoing to improve these metrics for semi-analytic reconstruction.

Reconstruction of flux in the context of multi-dimensional, vector conservation laws including effects of thermochemical nonequilibrium in the Navier-Stokes equations is developed as an embedded implicit solution. Programming the implicit formulation at the centroid of a tetrahedron involves significant changes to the basic structure of existing codes and has not yet been completed for $3 \mathrm{D}$ cases. The $1 \mathrm{D}$ nozzle solutions demonstrate that the approach can be used to capture strong discontinuities with good retention of accuracy. The flexibility to provide truly three-dimensional reconstruction as well as the unified formulation of viscous and inviscid flux provides motivation 
for continued development.

The approach taken throughout has been to linearize with respect to characteristic variable $\mathbf{q}^{\prime}$. Yet review of a rich tradition of analytic solutions in boundary-layer theory (e.g Blasius solution) shows that transformations of both independent variables (Howarth-Dorodnitzyn) and dependent variables have been introduced to generate solutions. ${ }^{14}$ One may speculate that lessons learned in that tradition may be exploited to provide better reconstruction in the algorithms developed here.

\section{References}

${ }^{1}$ Gnoffo, P. A., "Computational Fluid dynamics Technology for Hypersonic Applications," AIAA Paper 20033259, June 2003.

${ }^{2}$ Gnoffo, P. A. and White, J. A., "Computational Aerothermodynamic Simulation Issues on Unstructured Grids," AIAA Paper 2004-2371, June 2004.

${ }^{3}$ van Leer, B., "Towards the Ultimate Conservative Scheme. V. A Second-Order Sequel to Godunov's Method," J. Comput. Phys., Vol. 32, 1979, pp. 101-136.

${ }^{4}$ Harten, A., Lax, P. D., and van Leer, B., "On Upstream Differencing and Godunov-Type Schemes for Hyperbolic Conservation Laws," SIAM Review, Vol. 25, No. 1, January 1983, pp. 35-61.

${ }^{5}$ Roe, P. L., "Approximate Riemann Solvers, Parameter Vectors, and Difference Schemes," J. Comput. Phys., Vol. 43, No. 2, Oct. 1981, pp. 357-372.

${ }^{6}$ Godunov, S. K., "A Difference Method for the Numerical Calculation of Discontinuous Solutions of Hydrodynamic Equations," Matematichaskiy Sbornik, Vol. 47(89), No. 3, March 1959, pp. 271-306.

${ }^{7}$ Gnoffo, P. A. and Inger, G. R., "Analytic Corrections to Computational Heating Predictions Accounting for Changes in Surface Catalysis," J. Spacecraft and Rockets, Vol. 35, No. 4, July 1998, pp. 417-423.

${ }^{8}$ Inger, G. R. and Gnoffo, P. A., "Analytical and Computational Study of Wall Temperature Jumps in Supersonic Flow," AIAA J., Vol. 39, No. 1, Jan. 2001, pp. 79-87.

${ }^{9}$ Inger, G. R., "Triple Deck Theory of Supersonic Laminar Viscous-Inviscid Interaction Due to Wall temperature Jumps," NCC-1-02016 final report, Iowa State University, 2005.

${ }^{10}$ van Leer, B., "On the Relation Between the Upwind-Differencing Schemes of Godunov, Enquist-Osher and Roe," SIAM J. Sci. Stat. Comput., Vol. 5, No. 1, March 1984, pp. 1-20.

${ }^{11}$ Yee, H. C., "On Symmetric and Upwind TVD Schemes," NASA TM 88325, 1986.

${ }^{12}$ Yee, H. C., "Construction of Explicit and Implicit Symmetric TVD Schemes and Their Applications," J. Comput. Phys., Vol. 68, January 1987, pp. 151-179.

${ }^{13}$ Anderson, W. K. and Bonhaus, D. L., "An Implicit Upwind Algorithm for Computing Turbulent Flows on Unstructured Grids," Comp. and Fluids, Vol. 23, No. 1, Jan. 1994, pp. 1-21.

${ }^{14}$ Anderson, J. D., Hypersonic and High Temperature Gas Dynamics, McGraw-Hill, Inc., 1989. 
Table 1. Constant Wave Speed - Shock - Odd Number of Cells

\begin{tabular}{|c|c|c|c|c|c|c|c|}
\hline$\nu$ & cells & $\left|u-u_{e}\right|$ & $\left|u_{R o e}-u_{e}\right|$ & iter. & $L_{2}$ & $L_{2, \text { Roe }}$ & $\Delta x / \nu$ \\
\hline \multirow{6}{*}{100.} & 5 & $1.0 \mathrm{E}-07$ & $9.4 \mathrm{E}-07$ & 3 & $8.5 \mathrm{E}-11$ & $2.5 \mathrm{E}-10$ & 4.0E-03 \\
\hline & 9 & 3.3E-08 & $2.8 \mathrm{E}-07$ & 4 & $1.6 \mathrm{E}-13$ & $1.0 \mathrm{E}-12$ & 2.2E-03 \\
\hline & 17 & $9.6 \mathrm{E}-09$ & $6.5 \mathrm{E}-08$ & 4 & $1.2 \mathrm{E}-12$ & $3.0 \mathrm{E}-12$ & 1.2E-03 \\
\hline & 33 & 2.5E-09 & $1.4 \mathrm{E}-08$ & 4 & $1.2 \mathrm{E}-11$ & $1.6 \mathrm{E}-11$ & $6.1 \mathrm{E}-04$ \\
\hline & 65 & $6.6 \mathrm{E}-10$ & $3.2 \mathrm{E}-09$ & 5 & $1.6 \mathrm{E}-12$ & $4.7 \mathrm{E}-13$ & $3.1 \mathrm{E}-04$ \\
\hline & 129 & $1.7 \mathrm{E}-10$ & $7.4 \mathrm{E}-10$ & 5 & $6.7 \mathrm{E}-12$ & $9.4 \mathrm{E}-12$ & $1.6 \mathrm{E}-04$ \\
\hline \multirow{6}{*}{1.00} & 5 & $1.2 \mathrm{E}-03$ & $9.2 \mathrm{E}-03$ & 7 & $7.4 \mathrm{E}-11$ & 8.7E-10 & $4.0 \mathrm{E}-01$ \\
\hline & 9 & $3.7 \mathrm{E}-04$ & $2.8 \mathrm{E}-03$ & 8 & $7.6 \mathrm{E}-11$ & $4.5 \mathrm{E}-09$ & $2.2 \mathrm{E}-01$ \\
\hline & 17 & $1.1 \mathrm{E}-04$ & $6.6 \mathrm{E}-04$ & 10 & 3.7E-11 & $3.8 \mathrm{E}-10$ & 1.2E-01 \\
\hline & 33 & $2.8 \mathrm{E}-05$ & $1.4 \mathrm{E}-04$ & 13 & $2.9 \mathrm{E}-11$ & $7.1 \mathrm{E}-11$ & $6.1 \mathrm{E}-02$ \\
\hline & 65 & $7.2 \mathrm{E}-06$ & $3.2 \mathrm{E}-05$ & 18 & $2.6 \mathrm{E}-11$ & $3.5 \mathrm{E}-11$ & 3.1E-02 \\
\hline & 129 & $1.8 \mathrm{E}-06$ & 7.3E-06 & 26 & $5.4 \mathrm{E}-11$ & $5.9 \mathrm{E}-11$ & $1.6 \mathrm{E}-02$ \\
\hline \multirow{6}{*}{0.01} & 5 & $1.3 \mathrm{E}-09$ & $4.1 \mathrm{E}-02$ & 61 & $7.8 \mathrm{E}-11$ & 8.0E-11 & $4.0 \mathrm{E}+01$ \\
\hline & 9 & $1.3 \mathrm{E}-05$ & $3.9 \mathrm{E}-02$ & 60 & $8.2 \mathrm{E}-11$ & $2.8 \mathrm{E}-12$ & $2.2 \mathrm{E}+01$ \\
\hline & 17 & $1.3 \mathrm{E}-03$ & $3.7 \mathrm{E}-02$ & 60 & $7.3 \mathrm{E}-11$ & $1.0 \mathrm{E}-10$ & $1.2 \mathrm{E}+01$ \\
\hline & 33 & $1.1 \mathrm{E}-02$ & $2.5 \mathrm{E}-02$ & 60 & $7.5 \mathrm{E}-11$ & $1.9 \mathrm{E}-12$ & $6.1 \mathrm{E}+00$ \\
\hline & 65 & $8.1 \mathrm{E}-03$ & $1.1 \mathrm{E}-02$ & 23 & 8.7E-11 & $1.6 \mathrm{E}-06$ & $3.1 \mathrm{E}+00$ \\
\hline & 129 & $1.2 \mathrm{E}-03$ & $3.2 \mathrm{E}-03$ & 32 & $6.5 \mathrm{E}-11$ & 3.7E-09 & $1.6 \mathrm{E}+00$ \\
\hline \multirow{6}{*}{$10^{-4}$} & 5 & $2.0 \mathrm{E}-09$ & $4.0 \mathrm{E}-04$ & 61 & $7.8 \mathrm{E}-11$ & $9.6 \mathrm{E}-17$ & $4.0 \mathrm{E}+03$ \\
\hline & 9 & $1.1 \mathrm{E}-09$ & $4.0 \mathrm{E}-04$ & 61 & $7.2 \mathrm{E}-11$ & $9.1 \mathrm{E}-12$ & $2.2 \mathrm{E}+03$ \\
\hline & 17 & $5.9 \mathrm{E}-10$ & $4.0 \mathrm{E}-04$ & 58 & $9.3 \mathrm{E}-11$ & $1.8 \mathrm{E}-11$ & $1.2 \mathrm{E}+03$ \\
\hline & 33 & $3.1 \mathrm{E}-10$ & $4.0 \mathrm{E}-04$ & 59 & 8.7E-11 & $3.8 \mathrm{E}-11$ & $6.1 \mathrm{E}+02$ \\
\hline & 65 & $1.6 \mathrm{E}-10$ & $4.0 \mathrm{E}-04$ & 58 & $9.1 \mathrm{E}-11$ & $3.8 \mathrm{E}-10$ & $3.1 \mathrm{E}+02$ \\
\hline & 129 & $7.7 \mathrm{E}-11$ & 4.0E-04 & 58 & $7.8 \mathrm{E}-11$ & 7.1E-10 & $1.6 \mathrm{E}+02$ \\
\hline \multirow{6}{*}{$10^{-6}$} & 5 & $2.0 \mathrm{E}-09$ & $4.0 \mathrm{E}-06$ & 61 & $7.8 \mathrm{E}-11$ & $7.2 \mathrm{E}-17$ & $4.0 \mathrm{E}+05$ \\
\hline & 9 & $1.1 \mathrm{E}-09$ & $4.0 \mathrm{E}-06$ & 61 & $7.2 \mathrm{E}-11$ & $3.0 \mathrm{E}-16$ & $2.2 \mathrm{E}+05$ \\
\hline & 17 & $5.9 \mathrm{E}-10$ & $4.0 \mathrm{E}-06$ & 58 & $9.3 \mathrm{E}-11$ & $3.8 \mathrm{E}-16$ & $1.2 \mathrm{E}+05$ \\
\hline & 33 & $3.1 \mathrm{E}-10$ & $4.0 \mathrm{E}-06$ & 59 & $8.7 \mathrm{E}-11$ & $8.4 \mathrm{E}-14$ & $6.1 \mathrm{E}+04$ \\
\hline & 65 & $1.6 \mathrm{E}-10$ & $4.0 \mathrm{E}-06$ & 58 & $9.1 \mathrm{E}-11$ & $4.6 \mathrm{E}-13$ & $3.1 \mathrm{E}+04$ \\
\hline & 129 & $7.7 \mathrm{E}-11$ & $4.0 \mathrm{E}-06$ & 58 & $8.1 \mathrm{E}-11$ & $1.2 \mathrm{E}-11$ & $1.6 \mathrm{E}+04$ \\
\hline \multirow{6}{*}{$10^{-8}$} & 5 & 2.0E-09 & $4.0 \mathrm{E}-08$ & 61 & $7.8 \mathrm{E}-11$ & $9.6 \mathrm{E}-17$ & $4.0 \mathrm{E}+07$ \\
\hline & 9 & $1.1 \mathrm{E}-09$ & $4.0 \mathrm{E}-08$ & 61 & $7.2 \mathrm{E}-11$ & $6.7 \mathrm{E}-16$ & $2.2 \mathrm{E}+07$ \\
\hline & 17 & $5.9 \mathrm{E}-10$ & $4.0 \mathrm{E}-08$ & 58 & $9.3 \mathrm{E}-11$ & $5.7 \mathrm{E}-16$ & $1.2 \mathrm{E}+07$ \\
\hline & 33 & $3.1 \mathrm{E}-10$ & $4.0 \mathrm{E}-08$ & 59 & $8.7 \mathrm{E}-11$ & $6.7 \mathrm{E}-16$ & $6.1 \mathrm{E}+06$ \\
\hline & 65 & $1.6 \mathrm{E}-10$ & $4.0 \mathrm{E}-08$ & 58 & $9.1 \mathrm{E}-11$ & $7.2 \mathrm{E}-16$ & $3.1 \mathrm{E}+06$ \\
\hline & 129 & 7.7E-11 & $4.0 \mathrm{E}-08$ & 58 & $8.1 \mathrm{E}-11$ & $1.9 \mathrm{E}-15$ & $1.6 \mathrm{E}+06$ \\
\hline
\end{tabular}

34 of 52 
Table 2. End Point Wave Speed - Shock - Odd Number of Cells - $\phi=1$

\begin{tabular}{|c|c|c|c|c|c|c|c|}
\hline$\nu$ & cells & $\left|u-u_{e}\right|$ & $\left|u_{R o e}-u_{e}\right|$ & iter. & $L_{2}$ & $L_{2, \text { Roe }}$ & $\Delta x / \nu$ \\
\hline \multirow{6}{*}{100.} & 5 & $1.0 \mathrm{E}-07$ & $9.4 \mathrm{E}-07$ & 3 & $8.5 \mathrm{E}-11$ & $2.5 \mathrm{E}-10$ & 4.0E-03 \\
\hline & 9 & 3.3E-08 & $2.8 \mathrm{E}-07$ & 4 & $2.3 \mathrm{E}-13$ & $1.0 \mathrm{E}-12$ & 2.2E-03 \\
\hline & 17 & 9.6E-09 & $6.5 \mathrm{E}-08$ & 4 & $1.1 \mathrm{E}-12$ & $3.0 \mathrm{E}-12$ & $1.2 \mathrm{E}-03$ \\
\hline & 33 & 2.5E-09 & $1.4 \mathrm{E}-08$ & 4 & $1.3 \mathrm{E}-11$ & $1.6 \mathrm{E}-11$ & $6.1 \mathrm{E}-04$ \\
\hline & 65 & $6.6 \mathrm{E}-10$ & $3.2 \mathrm{E}-09$ & 5 & $1.1 \mathrm{E}-12$ & $4.7 \mathrm{E}-13$ & 3.1E-04 \\
\hline & 129 & $1.7 \mathrm{E}-10$ & $7.4 \mathrm{E}-10$ & 5 & $1.4 \mathrm{E}-11$ & $9.4 \mathrm{E}-12$ & $1.6 \mathrm{E}-04$ \\
\hline \multirow{6}{*}{1.00} & 5 & $1.3 \mathrm{E}-03$ & $9.2 \mathrm{E}-03$ & 7 & $8.3 \mathrm{E}-11$ & 8.7E-10 & $4.0 \mathrm{E}-01$ \\
\hline & 9 & $3.8 \mathrm{E}-04$ & $2.8 \mathrm{E}-03$ & 8 & 7.7E-11 & 4.5E-09 & 2.2E-01 \\
\hline & 17 & $1.1 \mathrm{E}-04$ & $6.6 \mathrm{E}-04$ & 10 & $3.7 \mathrm{E}-11$ & $3.8 \mathrm{E}-10$ & $1.2 \mathrm{E}-01$ \\
\hline & 33 & $2.8 \mathrm{E}-05$ & $1.4 \mathrm{E}-04$ & 13 & $2.9 \mathrm{E}-11$ & $7.1 \mathrm{E}-11$ & $6.1 \mathrm{E}-02$ \\
\hline & 65 & 7.2E-06 & $3.2 \mathrm{E}-05$ & 18 & $2.6 \mathrm{E}-11$ & $3.5 \mathrm{E}-11$ & 3.1E-02 \\
\hline & 129 & $1.8 \mathrm{E}-06$ & 7.3E-06 & 26 & $5.4 \mathrm{E}-11$ & $5.9 \mathrm{E}-11$ & $1.6 \mathrm{E}-02$ \\
\hline \multirow{6}{*}{0.01} & 5 & 1.1E-03 & 4.1E-02 & 12 & $2.2 \mathrm{E}-11$ & 1.0E-09 & $4.0 \mathrm{E}+01$ \\
\hline & 9 & 5.7E-04 & 3.9E-02 & 13 & $1.8 \mathrm{E}-11$ & $1.8 \mathrm{E}-05$ & $2.2 \mathrm{E}+01$ \\
\hline & 17 & $1.0 \mathrm{E}-03$ & 3.7E-02 & 14 & $4.4 \mathrm{E}-11$ & $6.8 \mathrm{E}-05$ & $1.2 \mathrm{E}+01$ \\
\hline & 33 & 1.0E-02 & 2.5E-02 & 18 & $2.4 \mathrm{E}-11$ & $1.7 \mathrm{E}-05$ & $6.1 \mathrm{E}+00$ \\
\hline & 65 & $9.7 \mathrm{E}-03$ & $1.1 \mathrm{E}-02$ & 24 & $2.6 \mathrm{E}-11$ & $6.8 \mathrm{E}-07$ & $3.1 \mathrm{E}+0 \mathrm{c}$ \\
\hline & 129 & $1.5 \mathrm{E}-03$ & $3.2 \mathrm{E}-03$ & 32 & $6.8 \mathrm{E}-11$ & 3.7E-09 & $1.6 \mathrm{E}+00$ \\
\hline \multirow{6}{*}{$10^{-4}$} & 5 & 1.1E-03 & $4.0 \mathrm{E}-04$ & 12 & $2.2 \mathrm{E}-11$ & $1.4 \mathrm{E}-10$ & $4.0 \mathrm{E}+03$ \\
\hline & 9 & 5.8E-04 & $4.0 \mathrm{E}-04$ & 13 & $1.8 \mathrm{E}-11$ & $2.8 \mathrm{E}-07$ & $2.2 \mathrm{E}+03$ \\
\hline & 17 & $3.1 \mathrm{E}-04$ & $4.0 \mathrm{E}-04$ & 14 & $4.6 \mathrm{E}-11$ & 4.3E-07 & $1.2 \mathrm{E}+03$ \\
\hline & 33 & $1.6 \mathrm{E}-04$ & $4.0 \mathrm{E}-04$ & 18 & $2.4 \mathrm{E}-11$ & $1.3 \mathrm{E}-06$ & $6.1 \mathrm{E}+02$ \\
\hline & 65 & 8.1E-05 & $4.0 \mathrm{E}-04$ & 23 & $2.9 \mathrm{E}-11$ & $1.3 \mathrm{E}-06$ & $3.1 \mathrm{E}+02$ \\
\hline & 129 & $4.1 \mathrm{E}-05$ & $4.0 \mathrm{E}-04$ & 31 & $3.0 \mathrm{E}-11$ & $1.3 \mathrm{E}-06$ & $1.6 \mathrm{E}+02$ \\
\hline \multirow{6}{*}{$10^{-6}$} & 5 & $1.1 \mathrm{E}-03$ & $4.0 \mathrm{E}-06$ & 12 & $2.2 \mathrm{E}-11$ & $3.1 \mathrm{E}-10$ & $4.0 \mathrm{E}+05$ \\
\hline & 9 & $5.8 \mathrm{E}-04$ & $4.0 \mathrm{E}-06$ & 13 & $1.8 \mathrm{E}-11$ & $4.4 \mathrm{E}-10$ & $2.2 \mathrm{E}+05$ \\
\hline & 17 & $3.1 \mathrm{E}-04$ & $4.0 \mathrm{E}-06$ & 14 & $4.6 \mathrm{E}-11$ & 6.6E-09 & $1.2 \mathrm{E}+05$ \\
\hline & 33 & $1.6 \mathrm{E}-04$ & $4.0 \mathrm{E}-06$ & 18 & $2.4 \mathrm{E}-11$ & $5.0 \mathrm{E}-10$ & $6.1 \mathrm{E}+04$ \\
\hline & 65 & 8.1E-05 & $4.0 \mathrm{E}-06$ & 23 & $2.9 \mathrm{E}-11$ & $5.0 \mathrm{E}-10$ & $3.1 \mathrm{E}+04$ \\
\hline & 129 & $4.1 \mathrm{E}-05$ & 4.0E-06 & 31 & $3.0 \mathrm{E}-11$ & 2.7E-09 & $1.6 \mathrm{E}+04$ \\
\hline \multirow{6}{*}{$10^{-8}$} & 5 & 1.1E-03 & $3.9 \mathrm{E}-08$ & 12 & $2.2 \mathrm{E}-11$ & $2.4 \mathrm{E}-08$ & $4.0 \mathrm{E}+07$ \\
\hline & 9 & $5.8 \mathrm{E}-04$ & $4.0 \mathrm{E}-08$ & 13 & $1.8 \mathrm{E}-11$ & $1.8 \mathrm{E}-10$ & $2.2 \mathrm{E}+07$ \\
\hline & 17 & $3.1 \mathrm{E}-04$ & $4.1 \mathrm{E}-08$ & 14 & $4.6 \mathrm{E}-11$ & 7.0E-09 & $1.2 \mathrm{E}+07$ \\
\hline & 33 & $1.6 \mathrm{E}-04$ & $4.0 \mathrm{E}-08$ & 18 & $2.4 \mathrm{E}-11$ & $9.9 \mathrm{E}-10$ & $6.1 \mathrm{E}+06$ \\
\hline & 65 & 8.1E-05 & $4.0 \mathrm{E}-08$ & 23 & $2.9 \mathrm{E}-11$ & $1.3 \mathrm{E}-10$ & $3.1 \mathrm{E}+06$ \\
\hline & 129 & 4.1E-05 & $4.0 \mathrm{E}-08$ & 31 & $3.0 \mathrm{E}-11$ & $2.7 \mathrm{E}-12$ & $1.6 \mathrm{E}+06$ \\
\hline
\end{tabular}

35 of 52 
Table 3. End Point Wave Speed - Shock - Odd Number of Cells - $\phi=\phi(\bar{\phi})$

\begin{tabular}{|c|c|c|c|c|c|c|c|}
\hline$\nu$ & cells & $\left|u-u_{e}\right|$ & $\left|u_{\text {Roe }}-u_{e}\right|$ & iter. & $L_{2}$ & $L_{2, \text { Roe }}$ & $\Delta x / \nu$ \\
\hline \multirow{6}{*}{100} & 5 & $9.7 \mathrm{E}-08$ & $9.4 \mathrm{E}-07$ & 3 & $8.5 \mathrm{E}-11$ & $2.5 \mathrm{E}-10$ & 4.0E-03 \\
\hline & 9 & 3.3E-08 & $2.8 \mathrm{E}-07$ & 4 & $1.4 \mathrm{E}-13$ & $1.0 \mathrm{E}-12$ & $2.2 \mathrm{E}-03$ \\
\hline & 17 & 9.5E-09 & $6.5 \mathrm{E}-08$ & 4 & $1.2 \mathrm{E}-12$ & $3.0 \mathrm{E}-12$ & $1.2 \mathrm{E}-03$ \\
\hline & 33 & 2.5E-09 & $1.4 \mathrm{E}-08$ & 4 & $1.3 \mathrm{E}-11$ & $1.6 \mathrm{E}-11$ & $6.1 \mathrm{E}-04$ \\
\hline & 65 & $6.6 \mathrm{E}-10$ & $3.2 \mathrm{E}-09$ & 5 & $2.3 \mathrm{E}-13$ & $4.7 \mathrm{E}-13$ & $3.1 \mathrm{E}-04$ \\
\hline & 129 & $1.7 \mathrm{E}-10$ & $7.4 \mathrm{E}-10$ & 5 & 8.9E-12 & $9.4 \mathrm{E}-12$ & $1.6 \mathrm{E}-04$ \\
\hline \multirow{6}{*}{1.00} & 5 & $1.2 \mathrm{E}-03$ & $9.2 \mathrm{E}-03$ & 7 & $7.9 \mathrm{E}-11$ & $8.7 \mathrm{E}-10$ & 4.0E-01 \\
\hline & 9 & 3.7E-04 & $2.8 \mathrm{E}-03$ & 8 & 7.7E-11 & 4.5E-09 & 2.2E-01 \\
\hline & 17 & $1.1 \mathrm{E}-04$ & $6.6 \mathrm{E}-04$ & 10 & $3.7 \mathrm{E}-11$ & $3.8 \mathrm{E}-10$ & $1.2 \mathrm{E}-01$ \\
\hline & 33 & $2.8 \mathrm{E}-05$ & $1.4 \mathrm{E}-04$ & 13 & $2.9 \mathrm{E}-11$ & 7.1E-11 & $6.1 \mathrm{E}-02$ \\
\hline & 65 & $7.2 \mathrm{E}-06$ & $3.2 \mathrm{E}-05$ & 18 & $2.6 \mathrm{E}-11$ & $3.5 \mathrm{E}-11$ & 3.1E-02 \\
\hline & 129 & $1.8 \mathrm{E}-06$ & 7.3E-06 & 26 & $5.4 \mathrm{E}-11$ & $5.9 \mathrm{E}-11$ & 1.6E-02 \\
\hline \multirow{6}{*}{0.01} & 5 & 1.6E-01 & 4.1E-02 & 21 & 4.7E-11 & $1.1 \mathrm{E}-14$ & $4.0 \mathrm{E}+01$ \\
\hline & 9 & $9.1 \mathrm{E}-02$ & 3.9E-02 & 21 & $3.9 \mathrm{E}-11$ & $5.7 \mathrm{E}-07$ & $2.2 \mathrm{E}+01$ \\
\hline & 17 & 4.7E-02 & 3.7E-02 & 22 & $3.5 \mathrm{E}-11$ & $4.8 \mathrm{E}-06$ & $1.2 \mathrm{E}+01$ \\
\hline & 33 & $1.4 \mathrm{E}-02$ & $2.5 \mathrm{E}-02$ & 22 & $5.8 \mathrm{E}-11$ & $3.3 \mathrm{E}-06$ & $6.1 \mathrm{E}+00$ \\
\hline & 65 & $2.8 \mathrm{E}-03$ & $1.1 \mathrm{E}-02$ & 23 & $3.6 \mathrm{E}-11$ & $1.6 \mathrm{E}-06$ & $3.1 \mathrm{E}+00$ \\
\hline & 129 & $1.2 \mathrm{E}-03$ & $3.2 \mathrm{E}-03$ & 32 & $6.5 \mathrm{E}-11$ & 3.7E-09 & $1.6 \mathrm{E}+00$ \\
\hline \multirow{6}{*}{$10^{-4}$} & 5 & $1.6 \mathrm{E}-01$ & $4.0 \mathrm{E}-04$ & 21 & 4.7E-11 & $9.6 \mathrm{E}-17$ & $4.0 \mathrm{E}+03$ \\
\hline & 9 & $9.1 \mathrm{E}-02$ & $4.0 \mathrm{E}-04$ & 21 & $3.1 \mathrm{E}-11$ & $5.6 \mathrm{E}-08$ & $2.2 \mathrm{E}+03$ \\
\hline & 17 & $4.8 \mathrm{E}-02$ & $4.0 \mathrm{E}-04$ & 21 & $6.5 \mathrm{E}-11$ & $1.0 \mathrm{E}-07$ & $1.2 \mathrm{E}+03$ \\
\hline & 33 & $2.5 \mathrm{E}-02$ & $4.0 \mathrm{E}-04$ & 22 & $4.6 \mathrm{E}-11$ & $5.7 \mathrm{E}-07$ & $6.1 \mathrm{E}+02$ \\
\hline & 65 & $1.3 \mathrm{E}-02$ & $4.0 \mathrm{E}-04$ & 23 & $4.2 \mathrm{E}-11$ & $1.3 \mathrm{E}-06$ & $3.1 \mathrm{E}+02$ \\
\hline & 129 & $6.3 \mathrm{E}-03$ & $4.0 \mathrm{E}-04$ & 30 & $9.0 \mathrm{E}-11$ & $1.7 \mathrm{E}-06$ & $1.6 \mathrm{E}+02$ \\
\hline \multirow{6}{*}{$10^{-6}$} & 5 & $1.6 \mathrm{E}-01$ & $4.0 \mathrm{E}-06$ & 21 & 4.7E-11 & $7.2 \mathrm{E}-17$ & $4.0 \mathrm{E}+05$ \\
\hline & 9 & $9.1 \mathrm{E}-02$ & $4.0 \mathrm{E}-06$ & 21 & $3.1 \mathrm{E}-11$ & $2.9 \mathrm{E}-12$ & $2.2 \mathrm{E}+05$ \\
\hline & 17 & $4.8 \mathrm{E}-02$ & $4.0 \mathrm{E}-06$ & 21 & $6.5 \mathrm{E}-11$ & $3.5 \mathrm{E}-12$ & $1.2 \mathrm{E}+05$ \\
\hline & 33 & $2.5 \mathrm{E}-02$ & $4.0 \mathrm{E}-06$ & 22 & $4.6 \mathrm{E}-11$ & $1.4 \mathrm{E}-10$ & $6.1 \mathrm{E}+04$ \\
\hline & 65 & $1.3 \mathrm{E}-02$ & $4.0 \mathrm{E}-06$ & 23 & $4.3 \mathrm{E}-11$ & $5.0 \mathrm{E}-10$ & $3.1 \mathrm{E}+04$ \\
\hline & 129 & $6.3 \mathrm{E}-03$ & 4.0E-06 & 30 & $9.0 \mathrm{E}-11$ & 3.3E-09 & $1.6 \mathrm{E}+04$ \\
\hline \multirow{6}{*}{$10^{-8}$} & 5 & $1.6 \mathrm{E}-01$ & $4.0 \mathrm{E}-08$ & 21 & $4.7 \mathrm{E}-11$ & $9.6 \mathrm{E}-17$ & $4.0 \mathrm{E}+07$ \\
\hline & 9 & $9.1 \mathrm{E}-02$ & $4.0 \mathrm{E}-08$ & 21 & $3.1 \mathrm{E}-11$ & $1.1 \mathrm{E}-15$ & $2.2 \mathrm{E}+07$ \\
\hline & 17 & $4.8 \mathrm{E}-02$ & $4.0 \mathrm{E}-08$ & 21 & $6.5 \mathrm{E}-11$ & $4.3 \mathrm{E}-13$ & $1.2 \mathrm{E}+07$ \\
\hline & 33 & $2.5 \mathrm{E}-02$ & $4.0 \mathrm{E}-08$ & 22 & $4.6 \mathrm{E}-11$ & $9.5 \mathrm{E}-12$ & $6.1 \mathrm{E}+06$ \\
\hline & 65 & $1.3 \mathrm{E}-02$ & $4.0 \mathrm{E}-08$ & 23 & $4.3 \mathrm{E}-11$ & $1.3 \mathrm{E}-10$ & $3.1 \mathrm{E}+06$ \\
\hline & 129 & $6.3 \mathrm{E}-03$ & $4.0 \mathrm{E}-08$ & 30 & $9.0 \mathrm{E}-11$ & $5.8 \mathrm{E}-12$ & $1.6 \mathrm{E}+06$ \\
\hline
\end{tabular}

36 of 52 
Table 4. Constant Wave Speed - Shock - Even Number of Cells

\begin{tabular}{|c|c|c|c|c|c|c|c|}
\hline$\nu$ & cells & $\left|u-u_{e}\right|$ & $\left|u_{R o e}-u_{e}\right|$ & iter. & $L_{2}$ & $L_{2, \text { Roe }}$ & $\Delta x / \nu$ \\
\hline \multirow{6}{*}{100.} & 6 & $6.9 \mathrm{E}-08$ & $6.5 \mathrm{E}-07$ & 4 & $2.9 \mathrm{E}-14$ & $4.2 \mathrm{E}-13$ & 3.3E-03 \\
\hline & 12 & $1.9 \mathrm{E}-08$ & $1.4 \mathrm{E}-07$ & 4 & $6.3 \mathrm{E}-13$ & $1.6 \mathrm{E}-12$ & 1.7E-03 \\
\hline & 24 & 4.8E-09 & $2.9 \mathrm{E}-08$ & 4 & $4.1 \mathrm{E}-12$ & $6.6 \mathrm{E}-12$ & 8.3E-04 \\
\hline & 48 & $1.2 \mathrm{E}-09$ & $6.2 \mathrm{E}-09$ & 4 & $4.4 \mathrm{E}-11$ & $5.2 \mathrm{E}-11$ & 4.2E-04 \\
\hline & 96 & $3.0 \mathrm{E}-10$ & $1.4 \mathrm{E}-09$ & 5 & $5.0 \mathrm{E}-12$ & $2.6 \mathrm{E}-12$ & 2.1E-04 \\
\hline & 192 & $7.3 \mathrm{E}-11$ & $3.2 \mathrm{E}-10$ & 5 & $3.8 \mathrm{E}-11$ & $5.5 \mathrm{E}-11$ & $1.0 \mathrm{E}-04$ \\
\hline \multirow{6}{*}{1.00} & 6 & $7.8 \mathrm{E}-04$ & $6.5 \mathrm{E}-03$ & 8 & $6.1 \mathrm{E}-12$ & $6.4 \mathrm{E}-10$ & 3.3E-01 \\
\hline & 12 & $2.1 \mathrm{E}-04$ & $1.5 \mathrm{E}-03$ & 9 & $3.1 \mathrm{E}-11$ & $1.1 \mathrm{E}-09$ & 1.7E-01 \\
\hline & 24 & $5.3 \mathrm{E}-05$ & $3.0 \mathrm{E}-04$ & 11 & $7.5 \mathrm{E}-11$ & $3.1 \mathrm{E}-10$ & 8.3E-02 \\
\hline & 48 & $1.3 \mathrm{E}-05$ & $6.2 \mathrm{E}-05$ & 15 & $5.4 \mathrm{E}-11$ & $8.7 \mathrm{E}-11$ & $4.2 \mathrm{E}-02$ \\
\hline & 96 & $3.3 \mathrm{E}-06$ & $1.4 \mathrm{E}-05$ & 22 & $3.9 \mathrm{E}-11$ & $4.5 \mathrm{E}-11$ & 2.1E-02 \\
\hline & 192 & 8.3E-07 & $3.2 \mathrm{E}-06$ & 34 & $5.1 \mathrm{E}-11$ & $5.4 \mathrm{E}-11$ & $1.0 \mathrm{E}-02$ \\
\hline \multirow{6}{*}{0.01} & 6 & $3.8 \mathrm{E}-08$ & $1.4 \mathrm{E}-02$ & 12 & $4.6 \mathrm{E}-11$ & $1.8 \mathrm{E}-04$ & $3.3 \mathrm{E}+01$ \\
\hline & 12 & $2.0 \mathrm{E}-08$ & $1.4 \mathrm{E}-02$ & 13 & $2.6 \mathrm{E}-11$ & $1.3 \mathrm{E}-03$ & $1.7 \mathrm{E}+01$ \\
\hline & 24 & $5.7 \mathrm{E}-05$ & $1.4 \mathrm{E}-02$ & 16 & $2.5 \mathrm{E}-11$ & $2.5 \mathrm{E}-04$ & $8.3 \mathrm{E}+00$ \\
\hline & 48 & $1.3 \mathrm{E}-03$ & $1.2 \mathrm{E}-02$ & 20 & $5.0 \mathrm{E}-11$ & 8.5E-06 & $4.2 \mathrm{E}+00$ \\
\hline & 96 & $1.5 \mathrm{E}-03$ & $5.5 \mathrm{E}-03$ & 28 & $2.7 \mathrm{E}-11$ & $5.0 \mathrm{E}-08$ & $2.1 \mathrm{E}+00$ \\
\hline & 192 & $4.4 \mathrm{E}-04$ & $1.1 \mathrm{E}-03$ & 40 & $8.9 \mathrm{E}-11$ & $1.7 \mathrm{E}-11$ & $1.0 \mathrm{E}+00$ \\
\hline \multirow{6}{*}{$10^{-4}$} & 6 & $3.8 \mathrm{E}-08$ & $1.6 \mathrm{E}-04$ & 12 & $4.5 \mathrm{E}-11$ & $1.2 \mathrm{E}-04$ & $3.3 \mathrm{E}+03$ \\
\hline & 12 & $1.9 \mathrm{E}-08$ & $1.1 \mathrm{E}-04$ & 13 & $2.1 \mathrm{E}-11$ & $2.7 \mathrm{E}-04$ & $1.7 \mathrm{E}+03$ \\
\hline & 24 & $9.4 \mathrm{E}-09$ & $1.2 \mathrm{E}-04$ & 16 & $2.9 \mathrm{E}-11$ & $2.4 \mathrm{E}-04$ & $8.3 \mathrm{E}+02$ \\
\hline & 48 & 4.7E-09 & $1.3 \mathrm{E}-04$ & 20 & $5.5 \mathrm{E}-11$ & $3.0 \mathrm{E}-05$ & $4.2 \mathrm{E}+02$ \\
\hline & 96 & 2.3E-09 & $1.3 \mathrm{E}-04$ & 27 & $3.0 \mathrm{E}-11$ & $2.1 \mathrm{E}-06$ & $2.1 \mathrm{E}+02$ \\
\hline & 192 & $1.2 \mathrm{E}-09$ & $1.3 \mathrm{E}-04$ & 37 & $6.4 \mathrm{E}-11$ & $7.6 \mathrm{E}-06$ & $1.0 \mathrm{E}+02$ \\
\hline \multirow{6}{*}{$10^{-6}$} & 6 & $3.8 \mathrm{E}-08$ & $2.0 \mathrm{E}-06$ & 12 & $4.5 \mathrm{E}-11$ & $3.5 \mathrm{E}-06$ & $3.3 \mathrm{E}+05$ \\
\hline & 12 & $1.9 \mathrm{E}-08$ & $4.2 \mathrm{E}-07$ & 13 & $2.1 \mathrm{E}-11$ & $8.9 \mathrm{E}-06$ & $1.7 \mathrm{E}+05$ \\
\hline & 24 & $9.4 \mathrm{E}-09$ & 8.7E-07 & 16 & $2.9 \mathrm{E}-11$ & $9.0 \mathrm{E}-06$ & $8.3 \mathrm{E}+04$ \\
\hline & 48 & 4.7E-09 & $1.3 \mathrm{E}-06$ & 20 & $5.5 \mathrm{E}-11$ & $5.8 \mathrm{E}-07$ & $4.2 \mathrm{E}+04$ \\
\hline & 96 & 2.3E-09 & $1.3 \mathrm{E}-06$ & 27 & $3.0 \mathrm{E}-11$ & $8.5 \mathrm{E}-07$ & $2.1 \mathrm{E}+04$ \\
\hline & 192 & $1.2 \mathrm{E}-09$ & $1.3 \mathrm{E}-06$ & 37 & $6.4 \mathrm{E}-11$ & $1.5 \mathrm{E}-08$ & $1.0 \mathrm{E}+04$ \\
\hline \multirow{6}{*}{$10^{-8}$} & 6 & $3.8 \mathrm{E}-08$ & $5.4 \mathrm{E}-08$ & 12 & $4.5 \mathrm{E}-11$ & $4.4 \mathrm{E}-07$ & $3.3 \mathrm{E}+07$ \\
\hline & 12 & $1.9 \mathrm{E}-08$ & $2.1 \mathrm{E}-07$ & 13 & $2.1 \mathrm{E}-11$ & $2.2 \mathrm{E}-06$ & $1.7 \mathrm{E}+07$ \\
\hline & 24 & $9.4 \mathrm{E}-09$ & $6.8 \mathrm{E}-09$ & 16 & $2.9 \mathrm{E}-11$ & $3.9 \mathrm{E}-07$ & $8.3 \mathrm{E}+06$ \\
\hline & 48 & 4.7E-09 & $1.1 \mathrm{E}-08$ & 20 & $5.5 \mathrm{E}-11$ & $1.0 \mathrm{E}-07$ & $4.2 \mathrm{E}+06$ \\
\hline & 96 & 2.3E-09 & $1.2 \mathrm{E}-08$ & 27 & $3.0 \mathrm{E}-11$ & 7.7E-08 & $2.1 \mathrm{E}+06$ \\
\hline & 192 & $1.2 \mathrm{E}-09$ & $1.3 \mathrm{E}-08$ & 37 & $6.4 \mathrm{E}-11$ & 9.9E-09 & $1.0 \mathrm{E}+06$ \\
\hline
\end{tabular}

37 of 52 
Table 5. End Point Wave Speed - Shock - Even Number of Cells - $\phi=1$

\begin{tabular}{|c|c|c|c|c|c|c|c|}
\hline$\nu$ & cells & $\left|u-u_{e}\right|$ & $\left|u_{R o e}-u_{e}\right|$ & iter. & $L_{2}$ & $L_{2, \text { Roe }}$ & $\Delta x / \nu$ \\
\hline \multirow{6}{*}{100.} & 6 & $6.9 \mathrm{E}-08$ & $6.5 \mathrm{E}-07$ & 4 & $3.3 \mathrm{E}-14$ & $4.2 \mathrm{E}-13$ & 3.3E-03 \\
\hline & 12 & $1.9 \mathrm{E}-08$ & $1.4 \mathrm{E}-07$ & 4 & $4.8 \mathrm{E}-13$ & $1.6 \mathrm{E}-12$ & 1.7E-03 \\
\hline & 24 & 4.8E-09 & $2.9 \mathrm{E}-08$ & 4 & $3.8 \mathrm{E}-12$ & $6.6 \mathrm{E}-12$ & 8.3E-04 \\
\hline & 48 & $1.2 \mathrm{E}-09$ & $6.2 \mathrm{E}-09$ & 4 & $4.4 \mathrm{E}-11$ & $5.2 \mathrm{E}-11$ & 4.2E-04 \\
\hline & 96 & $3.0 \mathrm{E}-10$ & $1.4 \mathrm{E}-09$ & 5 & $1.3 \mathrm{E}-12$ & $2.6 \mathrm{E}-12$ & 2.1E-04 \\
\hline & 192 & $7.5 \mathrm{E}-11$ & $3.2 \mathrm{E}-10$ & 5 & $4.5 \mathrm{E}-11$ & $5.5 \mathrm{E}-11$ & 1.0E-04 \\
\hline \multirow{6}{*}{1.00} & 6 & $8.0 \mathrm{E}-04$ & $6.5 \mathrm{E}-03$ & 8 & $6.4 \mathrm{E}-12$ & $6.4 \mathrm{E}-10$ & $3.3 \mathrm{E}-01$ \\
\hline & 12 & $2.1 \mathrm{E}-04$ & $1.5 \mathrm{E}-03$ & 9 & $3.1 \mathrm{E}-11$ & $1.1 \mathrm{E}-09$ & $1.7 \mathrm{E}-01$ \\
\hline & 24 & $5.3 \mathrm{E}-05$ & $3.0 \mathrm{E}-04$ & 11 & $7.5 \mathrm{E}-11$ & $3.1 \mathrm{E}-10$ & 8.3E-02 \\
\hline & 48 & $1.3 \mathrm{E}-05$ & $6.2 \mathrm{E}-05$ & 15 & $5.4 \mathrm{E}-11$ & 8.7E-11 & 4.2E-02 \\
\hline & 96 & $3.3 \mathrm{E}-06$ & $1.4 \mathrm{E}-05$ & 22 & $3.9 \mathrm{E}-11$ & $4.5 \mathrm{E}-11$ & $2.1 \mathrm{E}-02$ \\
\hline & 192 & 8.3E-07 & $3.2 \mathrm{E}-06$ & 34 & $5.1 \mathrm{E}-11$ & $5.4 \mathrm{E}-11$ & 1.0E-02 \\
\hline \multirow{6}{*}{0.01} & 6 & $1.0 \mathrm{E}-01$ & $1.4 \mathrm{E}-02$ & 11 & $9.6 \mathrm{E}-11$ & $2.8 \mathrm{E}-04$ & $3.3 \mathrm{E}+01$ \\
\hline & 12 & $5.2 \mathrm{E}-02$ & $1.4 \mathrm{E}-02$ & 12 & $9.6 \mathrm{E}-11$ & $1.5 \mathrm{E}-03$ & $1.7 \mathrm{E}+01$ \\
\hline & 24 & $2.6 \mathrm{E}-02$ & $1.4 \mathrm{E}-02$ & 16 & $1.9 \mathrm{E}-11$ & $2.5 \mathrm{E}-04$ & $8.3 \mathrm{E}+00$ \\
\hline & 48 & $9.0 \mathrm{E}-03$ & $1.2 \mathrm{E}-02$ & 20 & $7.8 \mathrm{E}-11$ & $8.5 \mathrm{E}-06$ & $4.2 \mathrm{E}+00$ \\
\hline & 96 & $2.3 \mathrm{E}-03$ & $5.5 \mathrm{E}-03$ & 28 & $3.1 \mathrm{E}-11$ & $5.0 \mathrm{E}-08$ & $2.1 \mathrm{E}+00$ \\
\hline & 192 & $5.0 \mathrm{E}-04$ & $1.1 \mathrm{E}-03$ & 40 & $9.0 \mathrm{E}-11$ & $1.7 \mathrm{E}-11$ & $1.0 \mathrm{E}+00$ \\
\hline \multirow{6}{*}{$10^{-4}$} & 6 & $1.0 \mathrm{E}-01$ & $1.0 \mathrm{E}-04$ & 11 & $9.6 \mathrm{E}-11$ & $1.6 \mathrm{E}-04$ & $3.3 \mathrm{E}+03$ \\
\hline & 12 & $5.2 \mathrm{E}-02$ & $1.7 \mathrm{E}-04$ & 12 & $9.2 \mathrm{E}-11$ & $3.5 \mathrm{E}-04$ & $1.7 \mathrm{E}+03$ \\
\hline & 24 & $2.6 \mathrm{E}-02$ & $1.2 \mathrm{E}-04$ & 16 & $2.0 \mathrm{E}-11$ & $2.4 \mathrm{E}-04$ & $8.3 \mathrm{E}+02$ \\
\hline & 48 & $1.3 \mathrm{E}-02$ & $1.3 \mathrm{E}-04$ & 20 & $4.6 \mathrm{E}-11$ & $3.0 \mathrm{E}-05$ & $4.2 \mathrm{E}+02$ \\
\hline & 96 & $6.4 \mathrm{E}-03$ & $1.3 \mathrm{E}-04$ & 27 & $2.8 \mathrm{E}-11$ & $2.1 \mathrm{E}-06$ & $2.1 \mathrm{E}+02$ \\
\hline & 192 & $3.2 \mathrm{E}-03$ & $1.3 \mathrm{E}-04$ & 37 & $6.3 \mathrm{E}-11$ & 7.6E-06 & $1.0 \mathrm{E}+02$ \\
\hline \multirow{6}{*}{$10^{-6}$} & 6 & $1.0 \mathrm{E}-01$ & $4.4 \mathrm{E}-07$ & 11 & $9.6 \mathrm{E}-11$ & $3.8 \mathrm{E}-06$ & $3.3 \mathrm{E}+05$ \\
\hline & 12 & $5.2 \mathrm{E}-02$ & $2.5 \mathrm{E}-06$ & 12 & $9.2 \mathrm{E}-11$ & $1.5 \mathrm{E}-05$ & $1.7 \mathrm{E}+05$ \\
\hline & 24 & $2.6 \mathrm{E}-02$ & $8.7 \mathrm{E}-07$ & 16 & $2.0 \mathrm{E}-11$ & $9.0 \mathrm{E}-06$ & $8.3 \mathrm{E}+04$ \\
\hline & 48 & $1.3 \mathrm{E}-02$ & $1.3 \mathrm{E}-06$ & 20 & $4.6 \mathrm{E}-11$ & $5.8 \mathrm{E}-07$ & $4.2 \mathrm{E}+04$ \\
\hline & 96 & $6.4 \mathrm{E}-03$ & $1.3 \mathrm{E}-06$ & 27 & $2.8 \mathrm{E}-11$ & $8.5 \mathrm{E}-07$ & $2.1 \mathrm{E}+04$ \\
\hline & 192 & $3.2 \mathrm{E}-03$ & $1.3 \mathrm{E}-06$ & 37 & $6.3 \mathrm{E}-11$ & $1.5 \mathrm{E}-08$ & $1.0 \mathrm{E}+04$ \\
\hline \multirow{6}{*}{$10^{-8}$} & 6 & $1.0 \mathrm{E}-01$ & $2.7 \mathrm{E}-07$ & 11 & $9.6 \mathrm{E}-11$ & $1.3 \mathrm{E}-06$ & $3.3 \mathrm{E}+07$ \\
\hline & 12 & $5.2 \mathrm{E}-02$ & $3.1 \mathrm{E}-07$ & 12 & $9.2 \mathrm{E}-11$ & $8.2 \mathrm{E}-06$ & $1.7 \mathrm{E}+07$ \\
\hline & 24 & $2.6 \mathrm{E}-02$ & $6.8 \mathrm{E}-09$ & 16 & $2.0 \mathrm{E}-11$ & $3.9 \mathrm{E}-07$ & $8.3 \mathrm{E}+06$ \\
\hline & 48 & $1.3 \mathrm{E}-02$ & $1.1 \mathrm{E}-08$ & 20 & $4.6 \mathrm{E}-11$ & $1.0 \mathrm{E}-07$ & $4.2 \mathrm{E}+06$ \\
\hline & 96 & $6.4 \mathrm{E}-03$ & $1.2 \mathrm{E}-08$ & 27 & $2.8 \mathrm{E}-11$ & 7.7E-08 & $2.1 \mathrm{E}+06$ \\
\hline & 192 & $3.2 \mathrm{E}-03$ & $1.3 \mathrm{E}-08$ & 37 & $6.3 \mathrm{E}-11$ & $9.9 \mathrm{E}-09$ & $1.0 \mathrm{E}+06$ \\
\hline
\end{tabular}

38 of 52 
Table 6. End Point Wave Speed - Shock - Even Number of Cells - $\phi=\phi(\bar{\phi})$

\begin{tabular}{|c|c|c|c|c|c|c|c|}
\hline$\nu$ & cells & $\left|u-u_{e}\right|$ & $\left|u_{R o e}-u_{e}\right|$ & iter. & $L_{2}$ & $L_{2, \text { Roe }}$ & $\Delta x / \nu$ \\
\hline \multirow{6}{*}{100} & 6 & $6.6 \mathrm{E}-08$ & $6.5 \mathrm{E}-07$ & 4 & $4.4 \mathrm{E}-14$ & $4.2 \mathrm{E}-13$ & $3.3 \mathrm{E}-03$ \\
\hline & 12 & $1.9 \mathrm{E}-08$ & $1.4 \mathrm{E}-07$ & 4 & $4.0 \mathrm{E}-13$ & $1.6 \mathrm{E}-12$ & $1.7 \mathrm{E}-03$ \\
\hline & 24 & 4.8E-09 & $2.9 \mathrm{E}-08$ & 4 & $4.2 \mathrm{E}-12$ & $6.6 \mathrm{E}-12$ & 8.3E-04 \\
\hline & 48 & $1.2 \mathrm{E}-09$ & $6.2 \mathrm{E}-09$ & 4 & $4.5 \mathrm{E}-11$ & $5.2 \mathrm{E}-11$ & $4.2 \mathrm{E}-04$ \\
\hline & 96 & $3.0 \mathrm{E}-10$ & $1.4 \mathrm{E}-09$ & 5 & $3.0 \mathrm{E}-12$ & $2.6 \mathrm{E}-12$ & $2.1 \mathrm{E}-04$ \\
\hline & 192 & $7.6 \mathrm{E}-11$ & $3.2 \mathrm{E}-10$ & 5 & $5.3 \mathrm{E}-11$ & $5.5 \mathrm{E}-11$ & $1.0 \mathrm{E}-04$ \\
\hline \multirow{6}{*}{1.00} & 6 & $7.6 \mathrm{E}-04$ & $6.5 \mathrm{E}-03$ & 8 & $6.2 \mathrm{E}-12$ & $6.4 \mathrm{E}-10$ & 3.3E-01 \\
\hline & 12 & $2.1 \mathrm{E}-04$ & $1.5 \mathrm{E}-03$ & 9 & $3.1 \mathrm{E}-11$ & $1.1 \mathrm{E}-09$ & 1.7E-01 \\
\hline & 24 & $5.3 \mathrm{E}-05$ & $3.0 \mathrm{E}-04$ & 11 & $7.5 \mathrm{E}-11$ & $3.1 \mathrm{E}-10$ & 8.3E-02 \\
\hline & 48 & $1.3 \mathrm{E}-05$ & $6.2 \mathrm{E}-05$ & 15 & $5.4 \mathrm{E}-11$ & $8.7 \mathrm{E}-11$ & 4.2E-02 \\
\hline & 96 & $3.3 \mathrm{E}-06$ & $1.4 \mathrm{E}-05$ & 22 & $3.9 \mathrm{E}-11$ & $4.5 \mathrm{E}-11$ & $2.1 \mathrm{E}-02$ \\
\hline & 192 & 8.3E-07 & $3.2 \mathrm{E}-06$ & 34 & $5.1 \mathrm{E}-11$ & $5.4 \mathrm{E}-11$ & $1.0 \mathrm{E}-02$ \\
\hline \multirow{6}{*}{0.01} & 6 & $9.3 \mathrm{E}-02$ & $1.4 \mathrm{E}-02$ & 11 & $3.0 \mathrm{E}-11$ & $2.8 \mathrm{E}-04$ & $3.3 \mathrm{E}+01$ \\
\hline & 12 & 4.7E-02 & $1.4 \mathrm{E}-02$ & 12 & $5.5 \mathrm{E}-11$ & $1.5 \mathrm{E}-03$ & $1.7 \mathrm{E}+01$ \\
\hline & 24 & 2.3E-02 & $1.4 \mathrm{E}-02$ & 16 & $3.8 \mathrm{E}-11$ & $2.5 \mathrm{E}-04$ & $8.3 \mathrm{E}+00$ \\
\hline & 48 & $6.1 \mathrm{E}-03$ & $1.2 \mathrm{E}-02$ & 20 & $6.1 \mathrm{E}-11$ & $8.5 \mathrm{E}-06$ & $4.2 \mathrm{E}+00$ \\
\hline & 96 & $1.7 \mathrm{E}-03$ & $5.5 \mathrm{E}-03$ & 28 & $2.8 \mathrm{E}-11$ & $5.0 \mathrm{E}-08$ & $2.1 \mathrm{E}+00$ \\
\hline & 192 & $4.6 \mathrm{E}-04$ & $1.1 \mathrm{E}-03$ & 40 & $9.0 \mathrm{E}-11$ & $1.7 \mathrm{E}-11$ & $1.0 \mathrm{E}+00$ \\
\hline \multirow{6}{*}{$10^{-4}$} & 6 & $9.3 \mathrm{E}-02$ & $1.0 \mathrm{E}-04$ & 11 & $3.0 \mathrm{E}-11$ & $1.6 \mathrm{E}-04$ & $3.3 \mathrm{E}+03$ \\
\hline & 12 & 4.7E-02 & $1.7 \mathrm{E}-04$ & 12 & $4.0 \mathrm{E}-11$ & $3.5 \mathrm{E}-04$ & $1.7 \mathrm{E}+03$ \\
\hline & 24 & $2.3 \mathrm{E}-02$ & $1.2 \mathrm{E}-04$ & 16 & $1.9 \mathrm{E}-11$ & $2.4 \mathrm{E}-04$ & $8.3 \mathrm{E}+02$ \\
\hline & 48 & $1.2 \mathrm{E}-02$ & $1.3 \mathrm{E}-04$ & 20 & $4.5 \mathrm{E}-11$ & $3.0 \mathrm{E}-05$ & $4.2 \mathrm{E}+02$ \\
\hline & 96 & $5.8 \mathrm{E}-03$ & $1.3 \mathrm{E}-04$ & 27 & 2.7E-11 & $2.1 \mathrm{E}-06$ & $2.1 \mathrm{E}+02$ \\
\hline & 192 & $2.9 \mathrm{E}-03$ & $1.3 \mathrm{E}-04$ & 37 & $6.2 \mathrm{E}-11$ & $7.6 \mathrm{E}-06$ & $1.0 \mathrm{E}+02$ \\
\hline \multirow{6}{*}{$10^{-6}$} & 6 & $9.3 \mathrm{E}-02$ & $4.4 \mathrm{E}-07$ & 11 & $3.0 \mathrm{E}-11$ & $3.8 \mathrm{E}-06$ & $3.3 \mathrm{E}+05$ \\
\hline & 12 & 4.7E-02 & $2.5 \mathrm{E}-06$ & 12 & $4.0 \mathrm{E}-11$ & $1.5 \mathrm{E}-05$ & $1.7 \mathrm{E}+05$ \\
\hline & 24 & $2.3 \mathrm{E}-02$ & 8.7E-07 & 16 & $1.9 \mathrm{E}-11$ & $9.0 \mathrm{E}-06$ & $8.3 \mathrm{E}+04$ \\
\hline & 48 & $1.2 \mathrm{E}-02$ & $1.3 \mathrm{E}-06$ & 20 & $4.5 \mathrm{E}-11$ & $5.8 \mathrm{E}-07$ & $4.2 \mathrm{E}+04$ \\
\hline & 96 & $5.8 \mathrm{E}-03$ & $1.3 \mathrm{E}-06$ & 27 & $2.7 \mathrm{E}-11$ & $8.5 \mathrm{E}-07$ & $2.1 \mathrm{E}+04$ \\
\hline & 192 & $2.9 \mathrm{E}-03$ & $1.3 \mathrm{E}-06$ & 37 & $6.2 \mathrm{E}-11$ & $1.5 \mathrm{E}-08$ & $1.0 \mathrm{E}+04$ \\
\hline \multirow{6}{*}{$10^{-8}$} & 6 & $9.3 \mathrm{E}-02$ & $2.7 \mathrm{E}-07$ & 11 & $3.0 \mathrm{E}-11$ & $1.3 \mathrm{E}-06$ & $3.3 \mathrm{E}+07$ \\
\hline & 12 & 4.7E-02 & $3.1 \mathrm{E}-07$ & 12 & $4.0 \mathrm{E}-11$ & $8.2 \mathrm{E}-06$ & $1.7 \mathrm{E}+07$ \\
\hline & 24 & 2.3E-02 & $6.8 \mathrm{E}-09$ & 16 & $1.9 \mathrm{E}-11$ & $3.9 \mathrm{E}-07$ & $8.3 \mathrm{E}+06$ \\
\hline & 48 & $1.2 \mathrm{E}-02$ & $1.1 \mathrm{E}-08$ & 20 & $4.5 \mathrm{E}-11$ & $1.0 \mathrm{E}-07$ & $4.2 \mathrm{E}+06$ \\
\hline & 96 & $5.8 \mathrm{E}-03$ & $1.2 \mathrm{E}-08$ & 27 & $2.7 \mathrm{E}-11$ & 7.7E-08 & $2.1 \mathrm{E}+06$ \\
\hline & 192 & $2.9 \mathrm{E}-03$ & $1.3 \mathrm{E}-08$ & 37 & $6.2 \mathrm{E}-11$ & $9.9 \mathrm{E}-09$ & $1.0 \mathrm{E}+06$ \\
\hline
\end{tabular}

39 of 52 
Table 7. Constant Wave Speed - Boundary Layer - Odd Number of Cells

\begin{tabular}{|c|c|c|c|c|c|c|c|}
\hline$\nu$ & cells & $\left|u-u_{e}\right|$ & $\left|u_{R o e}-u_{e}\right|$ & iter. & $L_{2}$ & $L_{2, \text { Roe }}$ & $\Delta x / \nu$ \\
\hline \multirow{6}{*}{100.} & 5 & $1.0 \mathrm{E}-07$ & $2.3 \mathrm{E}-07$ & 3 & $8.4 \mathrm{E}-11$ & $2.5 \mathrm{E}-10$ & $4.0 \mathrm{E}-03$ \\
\hline & 9 & $3.3 \mathrm{E}-08$ & $6.0 \mathrm{E}-08$ & 4 & $2.0 \mathrm{E}-13$ & $1.0 \mathrm{E}-12$ & $2.2 \mathrm{E}-03$ \\
\hline & 17 & $9.5 \mathrm{E}-09$ & $1.9 \mathrm{E}-08$ & 4 & $1.4 \mathrm{E}-12$ & $3.0 \mathrm{E}-12$ & $1.2 \mathrm{E}-03$ \\
\hline & 33 & 2.5E-09 & $6.8 \mathrm{E}-09$ & 4 & $1.2 \mathrm{E}-11$ & $1.6 \mathrm{E}-11$ & $6.1 \mathrm{E}-04$ \\
\hline & 65 & $6.6 \mathrm{E}-10$ & 2.1E-09 & 5 & $7.5 \mathrm{E}-13$ & 4.7E-13 & $3.1 \mathrm{E}-04$ \\
\hline & 129 & $1.7 \mathrm{E}-10$ & $6.0 \mathrm{E}-10$ & 5 & $1.0 \mathrm{E}-11$ & $9.4 \mathrm{E}-12$ & $1.6 \mathrm{E}-04$ \\
\hline \multirow{6}{*}{1.00} & 5 & $8.6 \mathrm{E}-04$ & $1.6 \mathrm{E}-03$ & 7 & $3.5 \mathrm{E}-11$ & 7.2E-10 & 4.0E-01 \\
\hline & 9 & $2.9 \mathrm{E}-04$ & $6.3 \mathrm{E}-04$ & 8 & $3.5 \mathrm{E}-11$ & $1.8 \mathrm{E}-09$ & $2.2 \mathrm{E}-01$ \\
\hline & 17 & $8.5 \mathrm{E}-05$ & $2.1 \mathrm{E}-04$ & 10 & $1.4 \mathrm{E}-11$ & $1.3 \mathrm{E}-10$ & $1.2 \mathrm{E}-01$ \\
\hline & 33 & $2.3 \mathrm{E}-05$ & $7.0 \mathrm{E}-05$ & 12 & $6.8 \mathrm{E}-11$ & $1.5 \mathrm{E}-10$ & $6.1 \mathrm{E}-02$ \\
\hline & 65 & $5.9 \mathrm{E}-06$ & $2.1 \mathrm{E}-05$ & 17 & $2.8 \mathrm{E}-11$ & $3.6 \mathrm{E}-11$ & $3.1 \mathrm{E}-02$ \\
\hline & 129 & $1.5 \mathrm{E}-06$ & $5.9 \mathrm{E}-06$ & 21 & $4.6 \mathrm{E}-14$ & $2.2 \mathrm{E}-11$ & $1.6 \mathrm{E}-02$ \\
\hline \multirow{6}{*}{0.01} & 5 & $2.2 \mathrm{E}-02$ & 8.7E-03 & 26 & $3.1 \mathrm{E}-11$ & $4.4 \mathrm{E}-08$ & $4.0 \mathrm{E}+01$ \\
\hline & 9 & $1.8 \mathrm{E}-02$ & $1.3 \mathrm{E}-02$ & 27 & $5.3 \mathrm{E}-11$ & $2.0 \mathrm{E}-07$ & $2.2 \mathrm{E}+01$ \\
\hline & 17 & $1.2 \mathrm{E}-02$ & $9.6 \mathrm{E}-03$ & 28 & $1.0 \mathrm{E}-11$ & $1.3 \mathrm{E}-06$ & $1.2 \mathrm{E}+01$ \\
\hline & 33 & $6.2 \mathrm{E}-03$ & $5.2 \mathrm{E}-03$ & 27 & $1.5 \mathrm{E}-11$ & 8.0E-06 & $6.1 \mathrm{E}+00$ \\
\hline & 65 & $2.5 \mathrm{E}-03$ & $3.4 \mathrm{E}-03$ & 26 & $6.9 \mathrm{E}-11$ & $2.9 \mathrm{E}-05$ & $3.1 \mathrm{E}+00$ \\
\hline & 129 & $8.3 \mathrm{E}-04$ & $3.0 \mathrm{E}-03$ & 26 & $3.2 \mathrm{E}-11$ & $2.4 \mathrm{E}-06$ & $1.6 \mathrm{E}+00$ \\
\hline \multirow{6}{*}{$10^{-4}$} & 5 & $1.0 \mathrm{E}-03$ & $1.0 \mathrm{E}-01$ & 31 & $1.5 \mathrm{E}-11$ & $1.0 \mathrm{E}-02$ & $4.0 \mathrm{E}+03$ \\
\hline & 9 & $9.9 \mathrm{E}-04$ & $3.5 \mathrm{E}-04$ & 31 & $3.1 \mathrm{E}-11$ & $3.9 \mathrm{E}-04$ & $2.2 \mathrm{E}+03$ \\
\hline & 17 & $9.1 \mathrm{E}-04$ & $4.8 \mathrm{E}-04$ & 31 & $7.9 \mathrm{E}-11$ & $1.3 \mathrm{E}-03$ & $1.2 \mathrm{E}+03$ \\
\hline & 33 & $8.0 \mathrm{E}-04$ & $3.3 \mathrm{E}-04$ & 32 & $9.2 \mathrm{E}-11$ & $1.4 \mathrm{E}-04$ & $6.1 \mathrm{E}+02$ \\
\hline & 65 & $6.7 \mathrm{E}-04$ & $2.8 \mathrm{E}-04$ & 33 & $5.7 \mathrm{E}-11$ & $2.1 \mathrm{E}-03$ & $3.1 \mathrm{E}+02$ \\
\hline & 129 & $5.5 \mathrm{E}-04$ & $3.6 \mathrm{E}-04$ & 34 & $2.6 \mathrm{E}-11$ & $3.2 \mathrm{E}-03$ & $1.6 \mathrm{E}+02$ \\
\hline \multirow{6}{*}{$10^{-6}$} & 5 & $2.7 \mathrm{E}-04$ & $2.8 \mathrm{E}-02$ & 47 & $6.8 \mathrm{E}-11$ & 4.3E-04 & $4.0 \mathrm{E}+05$ \\
\hline & 9 & $1.5 \mathrm{E}-04$ & $1.9 \mathrm{E}+10$ & 48 & $7.3 \mathrm{E}-11$ & $4.8 \mathrm{E}+10$ & $2.2 \mathrm{E}+05$ \\
\hline & 17 & $8.0 \mathrm{E}-05$ & $1.2 \mathrm{E}+03$ & 49 & $6.9 \mathrm{E}-11$ & $3.6 \mathrm{E}+03$ & $1.2 \mathrm{E}+05$ \\
\hline & 33 & $4.4 \mathrm{E}-05$ & $3.4 \mathrm{E}-06$ & 50 & $6.4 \mathrm{E}-11$ & $1.3 \mathrm{E}-08$ & $6.1 \mathrm{E}+04$ \\
\hline & 65 & $2.7 \mathrm{E}-05$ & $3.4 \mathrm{E}-06$ & 51 & $6.1 \mathrm{E}-11$ & $1.7 \mathrm{E}-06$ & $3.1 \mathrm{E}+04$ \\
\hline & 129 & $1.4 \mathrm{E}-05$ & $2.5 \mathrm{E}-06$ & 38 & $2.5 \mathrm{E}-11$ & $1.0 \mathrm{E}-04$ & $1.6 \mathrm{E}+04$ \\
\hline \multirow{6}{*}{$10^{-8}$} & 5 & $2.7 \mathrm{E}-04$ & $2.7 \mathrm{E}-02$ & 47 & $7.0 \mathrm{E}-11$ & $4.8 \mathrm{E}-04$ & $4.0 \mathrm{E}+07$ \\
\hline & 9 & $1.5 \mathrm{E}-04$ & $3.8 \mathrm{E}+17$ & 48 & $7.4 \mathrm{E}-11$ & $9.9 \mathrm{E}+17$ & $2.2 \mathrm{E}+07$ \\
\hline & 17 & $7.9 \mathrm{E}-05$ & $1.4 \mathrm{E}+05$ & 49 & $7.0 \mathrm{E}-11$ & $6.4 \mathrm{E}+05$ & $1.2 \mathrm{E}+07$ \\
\hline & 33 & $4.1 \mathrm{E}-05$ & $1.3 \mathrm{E}+20$ & 50 & $6.5 \mathrm{E}-11$ & $7.2 \mathrm{E}+20$ & $6.1 \mathrm{E}+06$ \\
\hline & 65 & $2.1 \mathrm{E}-05$ & $3.6 \mathrm{E}+74$ & 51 & $6.1 \mathrm{E}-11$ & $3.9 \mathrm{E}+75$ & $3.1 \mathrm{E}+06$ \\
\hline & 129 & $1.0 \mathrm{E}-05$ & $1.6+141$ & 52 & $5.8 \mathrm{E}-11$ & $5.2+142$ & $1.6 \mathrm{E}+06$ \\
\hline
\end{tabular}

40 of 52 
Table 8. End Point Wave Speed - Boundary Layer - Odd Number of Cells

\begin{tabular}{|c|c|c|c|c|c|c|c|}
\hline$\nu$ & cells & $\left|u-u_{e}\right|$ & $\left|u_{R o e}-u_{e}\right|$ & iter. & $L_{2}$ & $L_{2, \text { Roe }}$ & $\Delta x / \nu$ \\
\hline \multirow{6}{*}{100.} & 5 & $1.0 \mathrm{E}-07$ & 2.3E-07 & 3 & $8.4 \mathrm{E}-11$ & $2.5 \mathrm{E}-10$ & $4.0 \mathrm{E}-03$ \\
\hline & 9 & $3.3 \mathrm{E}-08$ & $6.0 \mathrm{E}-08$ & 4 & $9.6 \mathrm{E}-14$ & $1.0 \mathrm{E}-12$ & $2.2 \mathrm{E}-03$ \\
\hline & 17 & 9.5E-09 & $1.9 \mathrm{E}-08$ & 4 & $9.8 \mathrm{E}-13$ & $3.0 \mathrm{E}-12$ & $1.2 \mathrm{E}-03$ \\
\hline & 33 & 2.5E-09 & $6.8 \mathrm{E}-09$ & 4 & $1.2 \mathrm{E}-11$ & $1.6 \mathrm{E}-11$ & $6.1 \mathrm{E}-04$ \\
\hline & 65 & $6.6 \mathrm{E}-10$ & 2.1E-09 & 5 & $1.1 \mathrm{E}-12$ & 4.7E-13 & $3.1 \mathrm{E}-04$ \\
\hline & 129 & $1.7 \mathrm{E}-10$ & $6.0 \mathrm{E}-10$ & 5 & $6.2 \mathrm{E}-12$ & $9.4 \mathrm{E}-12$ & $1.6 \mathrm{E}-04$ \\
\hline \multirow{6}{*}{1.00} & 5 & $8.2 \mathrm{E}-04$ & $1.6 \mathrm{E}-03$ & 7 & $3.9 \mathrm{E}-11$ & 7.2E-10 & 4.0E-01 \\
\hline & 9 & $2.9 \mathrm{E}-04$ & $6.3 \mathrm{E}-04$ & 8 & $3.5 \mathrm{E}-11$ & $1.8 \mathrm{E}-09$ & $2.2 \mathrm{E}-01$ \\
\hline & 17 & $8.5 \mathrm{E}-05$ & $2.1 \mathrm{E}-04$ & 10 & $1.4 \mathrm{E}-11$ & $1.3 \mathrm{E}-10$ & $1.2 \mathrm{E}-01$ \\
\hline & 33 & $2.3 \mathrm{E}-05$ & $7.0 \mathrm{E}-05$ & 12 & $6.8 \mathrm{E}-11$ & $1.5 \mathrm{E}-10$ & $6.1 \mathrm{E}-02$ \\
\hline & 65 & $5.9 \mathrm{E}-06$ & $2.1 \mathrm{E}-05$ & 17 & $2.8 \mathrm{E}-11$ & $3.6 \mathrm{E}-11$ & $3.1 \mathrm{E}-02$ \\
\hline & 129 & $1.5 \mathrm{E}-06$ & $5.9 \mathrm{E}-06$ & 21 & 8.3E-14 & $2.2 \mathrm{E}-11$ & $1.6 \mathrm{E}-02$ \\
\hline \multirow{6}{*}{0.01} & 5 & $8.9 \mathrm{E}-02$ & 8.7E-03 & 39 & $9.5 \mathrm{E}-11$ & $1.7 \mathrm{E}-09$ & $4.0 \mathrm{E}+01$ \\
\hline & 9 & $4.0 \mathrm{E}-02$ & $1.3 \mathrm{E}-02$ & 40 & $5.9 \mathrm{E}-11$ & $1.6 \mathrm{E}-10$ & $2.2 \mathrm{E}+01$ \\
\hline & 17 & $2.4 \mathrm{E}-03$ & $9.6 \mathrm{E}-03$ & 43 & $6.5 \mathrm{E}-11$ & $1.2 \mathrm{E}-12$ & $1.2 \mathrm{E}+01$ \\
\hline & 33 & $3.1 \mathrm{E}-03$ & $5.2 \mathrm{E}-03$ & 33 & $2.3 \mathrm{E}-11$ & 2.0E-08 & $6.1 \mathrm{E}+00$ \\
\hline & 65 & $1.7 \mathrm{E}-03$ & $3.4 \mathrm{E}-03$ & 27 & $1.8 \mathrm{E}-11$ & 4.0E-06 & $3.1 \mathrm{E}+00$ \\
\hline & 129 & $8.0 \mathrm{E}-04$ & $3.0 \mathrm{E}-03$ & 26 & $6.2 \mathrm{E}-11$ & $2.4 \mathrm{E}-06$ & $1.6 \mathrm{E}+00$ \\
\hline \multirow{6}{*}{$10^{-4}$} & 5 & $1.7 \mathrm{E}-01$ & $4.1 \mathrm{E}-01$ & 49 & $7.3 \mathrm{E}-11$ & $3.8 \mathrm{E}-02$ & $4.0 \mathrm{E}+03$ \\
\hline & 9 & $7.8 \mathrm{E}-02$ & $2.8 \mathrm{E}-04$ & 49 & $8.7 \mathrm{E}-11$ & $2.5 \mathrm{E}-06$ & $2.2 \mathrm{E}+03$ \\
\hline & 17 & $4.1 \mathrm{E}-02$ & $3.2 \mathrm{E}-04$ & 48 & $7.3 \mathrm{E}-11$ & $1.7 \mathrm{E}-05$ & $1.2 \mathrm{E}+03$ \\
\hline & 33 & $2.1 \mathrm{E}-02$ & $3.3 \mathrm{E}-04$ & 49 & $8.5 \mathrm{E}-11$ & $1.7 \mathrm{E}-06$ & $6.1 \mathrm{E}+02$ \\
\hline & 65 & $1.0 \mathrm{E}-02$ & $3.2 \mathrm{E}-04$ & 49 & $5.2 \mathrm{E}-11$ & $2.6 \mathrm{E}-05$ & $3.1 \mathrm{E}+02$ \\
\hline & 129 & $5.1 \mathrm{E}-03$ & $3.1 \mathrm{E}-04$ & 49 & $8.7 \mathrm{E}-11$ & $3.4 \mathrm{E}-05$ & $1.6 \mathrm{E}+02$ \\
\hline \multirow{6}{*}{$10^{-6}$} & 5 & $1.7 \mathrm{E}-01$ & $2.9 \mathrm{E}-02$ & 49 & $7.3 \mathrm{E}-11$ & 3.3E-04 & $4.0 \mathrm{E}+05$ \\
\hline & 9 & $7.9 \mathrm{E}-02$ & $5.2 \mathrm{E}+11$ & 51 & $8.0 \mathrm{E}-11$ & $1.6 \mathrm{E}+12$ & $2.2 \mathrm{E}+05$ \\
\hline & 17 & $4.2 \mathrm{E}-02$ & $8.6 \mathrm{E}+03$ & 52 & $5.6 \mathrm{E}-11$ & $2.8 \mathrm{E}+04$ & $1.2 \mathrm{E}+05$ \\
\hline & 33 & $2.2 \mathrm{E}-02$ & $3.4 \mathrm{E}-06$ & 51 & $9.3 \mathrm{E}-11$ & $9.9 \mathrm{E}-09$ & $6.1 \mathrm{E}+04$ \\
\hline & 65 & $1.1 \mathrm{E}-02$ & $3.5 \mathrm{E}-06$ & 52 & $9.7 \mathrm{E}-11$ & $1.3 \mathrm{E}-06$ & $3.1 \mathrm{E}+04$ \\
\hline & 129 & $5.5 \mathrm{E}-03$ & $3.5 \mathrm{E}-06$ & 53 & $9.0 \mathrm{E}-11$ & $2.6 \mathrm{E}-06$ & $1.6 \mathrm{E}+04$ \\
\hline \multirow{6}{*}{$10^{-8}$} & 5 & $1.7 \mathrm{E}-01$ & $2.7 \mathrm{E}-02$ & 49 & $7.3 \mathrm{E}-11$ & $3.7 \mathrm{E}-04$ & $4.0 \mathrm{E}+07$ \\
\hline & 9 & $7.9 \mathrm{E}-02$ & $7.9 \mathrm{E}+22$ & 51 & $8.0 \mathrm{E}-11$ & $1.6 \mathrm{E}+23$ & $2.2 \mathrm{E}+07$ \\
\hline & 17 & $4.2 \mathrm{E}-02$ & $2.1 \mathrm{E}+13$ & 63 & $9.0 \mathrm{E}-11$ & $8.4 \mathrm{E}+13$ & $1.2 \mathrm{E}+07$ \\
\hline & 33 & $2.2 \mathrm{E}-02$ & $1.6 \mathrm{E}+27$ & 53 & $6.3 \mathrm{E}-11$ & $1.3 \mathrm{E}+28$ & $6.1 \mathrm{E}+06$ \\
\hline & 65 & $1.1 \mathrm{E}-02$ & $1.1 \mathrm{E}+78$ & 53 & $9.7 \mathrm{E}-11$ & $1.5 \mathrm{E}+79$ & $3.1 \mathrm{E}+06$ \\
\hline & 129 & $5.5 \mathrm{E}-03$ & $4.8+152$ & 54 & 8.7E-11 & INF & $1.6 \mathrm{E}+06$ \\
\hline
\end{tabular}

41 of 52 
Table 9. End Point Wave Speed - Boundary Layer - Odd Number of Cells - $\phi=\phi(\bar{\phi})$

\begin{tabular}{cccccccc}
$\nu$ & cells & $\left|u-u_{e}\right|$ & $\left|u_{\text {Roe }}-u_{e}\right|$ & iter. & $L_{2}$ & $L_{2, \text { Roe }}$ & $\Delta x / \nu$ \\
\hline \multirow{6}{*}{100} & 5 & $9.7 \mathrm{E}-08$ & $2.3 \mathrm{E}-07$ & 3 & $8.5 \mathrm{E}-13$ & $4.4 \mathrm{E}-11$ & $4.0 \mathrm{E}-03$ \\
& 9 & $3.3 \mathrm{E}-08$ & $6.0 \mathrm{E}-08$ & 3 & $3.1 \mathrm{E}-12$ & $3.2 \mathrm{E}-10$ & $2.2 \mathrm{E}-03$ \\
& 17 & $9.5 \mathrm{E}-09$ & $1.9 \mathrm{E}-08$ & 3 & $1.4 \mathrm{E}-11$ & $3.1 \mathrm{E}-10$ & $1.2 \mathrm{E}-03$ \\
& 33 & $2.5 \mathrm{E}-09$ & $6.8 \mathrm{E}-09$ & 3 & $7.4 \mathrm{E}-11$ & $3.0 \mathrm{E}-10$ & $6.1 \mathrm{E}-04$ \\
& 65 & $6.6 \mathrm{E}-10$ & $2.1 \mathrm{E}-09$ & 4 & $6.4 \mathrm{E}-13$ & $2.6 \mathrm{E}-13$ & $3.1 \mathrm{E}-04$ \\
& 129 & $1.7 \mathrm{E}-10$ & $6.0 \mathrm{E}-10$ & 4 & $6.1 \mathrm{E}-12$ & $1.7 \mathrm{E}-12$ & $1.6 \mathrm{E}-04$ \\
& 5 & $7.9 \mathrm{E}-04$ & $1.6 \mathrm{E}-03$ & 5 & $1.6 \mathrm{E}-11$ & $1.5 \mathrm{E}-08$ & $4.0 \mathrm{E}-01$ \\
& 9 & $2.9 \mathrm{E}-04$ & $6.3 \mathrm{E}-04$ & 5 & $7.5 \mathrm{E}-11$ & $3.9 \mathrm{E}-07$ & $2.2 \mathrm{E}-01$ \\
1.00 & 17 & $8.5 \mathrm{E}-05$ & $2.1 \mathrm{E}-04$ & 6 & $7.9 \mathrm{E}-12$ & $1.1 \mathrm{E}-08$ & $1.2 \mathrm{E}-01$ \\
& 33 & $2.3 \mathrm{E}-05$ & $7.0 \mathrm{E}-05$ & 7 & $3.9 \mathrm{E}-12$ & $2.0 \mathrm{E}-10$ & $6.1 \mathrm{E}-02$ \\
& 65 & $5.9 \mathrm{E}-06$ & $2.1 \mathrm{E}-05$ & 8 & $8.2 \mathrm{E}-12$ & $3.3 \mathrm{E}-11$ & $3.1 \mathrm{E}-02$ \\
& 129 & $1.5 \mathrm{E}-06$ & $5.9 \mathrm{E}-06$ & 9 & $6.2 \mathrm{E}-11$ & $9.4 \mathrm{E}-11$ & $1.6 \mathrm{E}-02$ \\
& 5 & $1.3 \mathrm{E}-02$ & $8.5 \mathrm{E}-03$ & 21 & $4.7 \mathrm{E}-11$ & $3.5 \mathrm{E}-04$ & $4.0 \mathrm{E}+01$ \\
& 9 & $1.5 \mathrm{E}-02$ & $1.3 \mathrm{E}-02$ & 23 & $5.6 \mathrm{E}-11$ & $1.7 \mathrm{E}-06$ & $2.2 \mathrm{E}+01$ \\
& 17 & $1.6 \mathrm{E}-02$ & $9.6 \mathrm{E}-03$ & 58 & $7.1 \mathrm{E}-11$ & $4.2 \mathrm{E}-16$ & $1.2 \mathrm{E}+01$ \\
& 33 & $6.7 \mathrm{E}-04$ & $5.2 \mathrm{E}-03$ & 24 & $1.6 \mathrm{E}-11$ & $2.5 \mathrm{E}-09$ & $6.1 \mathrm{E}+00$ \\
& 65 & $1.8 \mathrm{E}-03$ & $3.4 \mathrm{E}-03$ & 24 & $3.1 \mathrm{E}-11$ & $1.8 \mathrm{E}-07$ & $3.1 \mathrm{E}+00$ \\
& 129 & $8.0 \mathrm{E}-04$ & $3.0 \mathrm{E}-03$ & 24 & $6.1 \mathrm{E}-12$ & $4.6 \mathrm{E}-07$ & $1.6 \mathrm{E}+00$ \\
& 33 & $7.5 \mathrm{E}-03$ & $2.5 \mathrm{E}+32$ & 43 & $8.3 \mathrm{E}-11$ & $1.8 \mathrm{E}+33$ & $6.1 \mathrm{E}+06$ \\
& 65 & $3.8 \mathrm{E}-03$ & $2.9 \mathrm{E}-08$ & 44 & $8.7 \mathrm{E}-11$ & $2.6 \mathrm{E}-07$ & $3.1 \mathrm{E}+06$ \\
& 129 & $1.9 \mathrm{E}-03$ & $1.2 \mathrm{E}+60$ & 45 & $9.4 \mathrm{E}-11$ & $3.7 \mathrm{E}+61$ & $1.6 \mathrm{E}+06$
\end{tabular}

42 of 52 
Table 10. Convergence Times for Qusai, One-Dimensional Nozzle

\begin{tabular}{|c|c|c|c|c|c|c|}
\hline Case & Cells & Total Time & Recon. Time & Iter. & $\frac{\text { Recon.Time }}{\text { Iter. }}$ & $\frac{\text { Recon.Time }}{\text { (Iter.)(Cell) }}$ \\
\hline & 36 & 1.67 & 1.05 & 273 & 0.003846 & 0.0001068 \\
\hline EPWS & 70 & 1.30 & 0.93 & 119 & 0.0078151 & 0.0001116 \\
\hline Exit Area $=20$ & 138 & 2.63 & 1.73 & 117 & 0.014786 & 0.0001071 \\
\hline \multirow[t]{3}{*}{$p_{\text {stag }}=10^{6}$} & 274 & 5.40 & 3.85 & 129 & 0.029844 & 0.0001089 \\
\hline & 546 & 13.79 & 9.92 & 164 & 0.060487 & 0.0001108 \\
\hline & 36 & 1.21 & 0.49 & 406 & 0.001206 & 0.0000335 \\
\hline Roe & 70 & 1.23 & 0.50 & 247 & 0.002024 & 0.0000289 \\
\hline Exit Area $=20$ & 138 & 2.63 & 1.01 & 268 & 0.003768 & 0.0000273 \\
\hline \multirow[t]{2}{*}{$p_{\text {stag }}=10^{6}$} & 274 & 7.65 & 2.78 & 401 & 0.006932 & 0.0000253 \\
\hline & 546 & 23.65 & 8.82 & 627 & 0.014067 & 0.0000258 \\
\hline
\end{tabular}

\section{Appendix A - One-Dimensional Reconstruction with Quadrature}

The methods in this section have not been fully explored in numerical tests but are a logical extension of the basic formulations in Sec. III. The methods require quadrature and tend not to be robust (or require careful application of limiters) in the limit of $\nu \rightarrow 0$. They are presented here to preserve ideas for future development of higher order methods.

\section{A. Multi-segment Wave Speed Approximation}

The multi-segment wave speed approximation breaks the interval $\left[x_{L}, x_{R}\right]$ into multiple segments. The wave speed across each segment is treated as a constant. This approach is essentially a solution by quadrature of Eq. 5. The linearized equation on segment $N$ is

$$
\lambda_{N} \frac{d q}{d x}=\nu_{N} \frac{d^{2} q}{d x^{2}}+s_{N}
$$

Following the derivation of Sec. A the transformed variable $\eta$ is defined.

$$
\begin{aligned}
\eta(x)= & \sum_{n=1}^{N}\left\{\int_{x_{n-1}-x_{0}}^{x_{n}-x_{0}} \exp \left[\frac{\lambda_{n-1}\left(x^{\prime}\right)}{\nu_{n-1}}\right] d x^{\prime}\right\}+\int_{x_{N}-x_{0}}^{x-x_{0}} \exp \left[\frac{\lambda_{N}\left(x^{\prime}\right)}{\nu_{N}}\right] d x^{\prime} \\
= & \sum_{n=1}^{N}\left\{\frac{\nu_{n-1}}{\lambda_{n-1}}\left(\exp \left[\frac{\lambda_{n-1}\left(x_{n}-x_{0}\right)}{\nu_{n-1}}\right]-\exp \left[\frac{\lambda_{n-1}\left(x_{n-1}-x_{0}\right)}{\nu_{n-1}}\right]\right)\right\} \\
& +\frac{\nu_{N}}{\lambda_{N}}\left(\exp \left[\frac{\lambda_{N}\left(x-x_{0}\right)}{\nu_{N}}\right]-\exp \left[\frac{\lambda_{N}\left(x_{N}-x_{0}\right)}{\nu_{N}}\right]\right) \\
\frac{d \eta}{d x}= & \exp \left[\frac{\lambda_{N}\left(x-x_{0}\right)}{\nu_{N}}\right] \\
\frac{d^{2} \eta}{d x^{2}}= & \frac{\lambda_{N}}{\nu_{N}} \frac{d \eta}{d x}
\end{aligned}
$$

The point $x$ lies in the segment $\left[x_{N+1}, x_{N}\right]$ (segment $N+1$ ). Segments are numbered consecutively from the interface $x_{0}$ moving to the endpoints $x_{L}$ or $x_{R}$. The analytic solution at each segment interface is $\mathrm{C} 1$ continuous. In the limit of $\lambda_{0} \rightarrow 0$ the mapping defined by Eq. 7 becomes

$$
\begin{array}{ccc}
\eta & = & x-x_{N}+\eta_{N} \\
\frac{d \eta}{d x} & = & 1 \\
\frac{d^{2} \eta}{d x^{2}} & = & 0
\end{array}
$$


where $\eta_{N}$ represents the value of the transformed variable through the first $N$ segments in Eq. 121.

$$
\eta_{N}=\sum_{n=1}^{N}\left\{\frac{\nu_{n-1}}{\lambda_{n-1}}\left(\exp \left[\frac{\lambda_{n-1}\left(x_{n}-x_{0}\right)}{\nu_{n-1}}\right]-\exp \left[\frac{\lambda_{n-1}\left(x_{n-1}-x_{0}\right)}{\nu_{n-1}}\right]\right)\right\}
$$

Substitution of Eq. 121 (or Eq. 122 if $\lambda_{N}=0$ ) into Eq. 120 yields the following.

$$
\frac{d^{2} q}{d \eta^{2}}=-\frac{s_{N}}{\nu_{N}}\left(\frac{d x}{d \eta}\right)^{2}
$$

The general solution to Eq. 124 is

$$
\begin{aligned}
q_{\text {general }}^{N} & =q_{N}+\left(\frac{d q}{d \eta}\right)_{N}\left(\eta-\eta_{N}\right) \\
& =q_{N}+\left(\frac{d q}{d x}\right)_{N}\left(\frac{d x}{d \eta}\right)_{N}\left(\eta-\eta_{N}\right) \\
& =q_{N}+\left(\frac{d q}{d x}\right)_{N} \frac{\nu_{N}}{\lambda_{N}}\left(\exp \left[\frac{\lambda_{N}\left(x-x_{0}\right)}{\nu_{N}}\right]-\exp \left[\frac{\lambda_{N}\left(x_{N}-x_{0}\right)}{\nu_{N}}\right]\right)
\end{aligned}
$$

The particular solution to Eq. 124 is derived as follows. First, integrate both sides of Eq. 124, returning to independent variable $x$ on the left hand side to simplify the integration.

$$
\begin{aligned}
\left(\frac{d q}{d \eta}\right)_{\text {particular }}^{N} & =-\frac{s_{N}}{\nu_{N}} \int_{\eta_{N}}^{\eta}\left(\frac{d x}{d \eta}\right)^{2} d \eta^{\prime} \\
& =-\frac{s_{N}}{\nu_{N}} \int_{x_{N}-x_{0}}^{x-x_{0}} \exp \left[-\frac{2 \lambda_{N}\left(x^{\prime}\right)}{\nu_{N}}\right] \frac{d \eta}{d x} d x^{\prime} \\
& =\frac{s_{N}}{\lambda_{N}}\left(\exp \left[-\frac{\lambda_{N}\left(x-x_{0}\right)}{\nu_{N}}\right]-\exp \left[-\frac{\lambda_{N}\left(x_{N}-x_{0}\right)}{\nu_{N}}\right]\right)
\end{aligned}
$$

Note that $\left(\frac{d q}{d \eta}\right)_{\text {particular, } N}^{N}=0$; consequently, the gradient $\left(\frac{d q}{d x}\right)_{N}$ is fully defined by the general solution for this case where $s, \lambda$, and $\nu$ are treated as a constants over the interval. Integrate both sides of Eq. 126 in the same manner to complete the derivation of the particular solution.

$$
\begin{aligned}
q_{\text {particular }}^{N}= & \int_{\eta_{N}}^{\eta}\left(\frac{d q}{d \eta}\right)_{\text {particular }} d \eta^{\prime} \\
= & \frac{s_{N}}{\lambda_{N}} \int_{x_{N}-x_{0}}^{x-x_{0}}\left(\exp \left[-\frac{\lambda_{N}\left(x^{\prime}\right)}{\nu_{N}}\right]-\exp \left[-\frac{\lambda_{N}\left(x_{N}-x_{0}\right)}{\nu_{N}}\right]\right) \frac{d \eta}{d x} d x^{\prime} \\
= & \frac{s_{N}}{\lambda_{N}} \int_{x_{N}-x_{0}}^{x-x_{0}}\left(1-\exp \left[\frac{\lambda_{N}\left(x^{\prime}-\left(x_{N}-x_{0}\right)\right)}{\nu_{N}}\right]\right) d x^{\prime} \\
= & \frac{s_{N}}{\lambda_{N}}\left[\left(x-x_{N}\right)-\frac{\nu_{N}}{\lambda_{N}}\left(\exp \left[-\frac{\lambda_{N}\left(x-x_{N}\right)}{\nu_{N}}\right]-1\right)\right] \\
= & \frac{s_{N}}{\lambda_{N}}\left[\left(x-x_{N}\right)\right. \\
& \left.-\frac{\nu_{N}}{\lambda_{N}} \exp \left[-\frac{\lambda_{N}\left(x_{N}-x_{0}\right)}{\nu_{N}}\right]\left(\exp \left[\frac{\lambda_{N}\left(x-x_{0}\right)}{\nu_{N}}\right]-\exp \left[\frac{\lambda_{N}\left(x_{N}-x_{0}\right)}{\nu_{N}}\right]\right)\right] \\
= & \frac{s_{N}}{\lambda_{N}}\left[\left(x-x_{N}\right)-\exp \left[-\frac{\lambda_{N}\left(x_{N}-x_{0}\right)}{\nu_{N}}\right]\left(\eta(x)-\eta_{N}\right)\right]
\end{aligned}
$$

In the limit as $\lambda_{0} \rightarrow 0$ the particular solution in Eq. 127 goes to the expected result

$$
q_{\text {particular }}^{N}=-\frac{s_{N}}{2 \nu_{N}}\left(x-x_{N}\right)^{2} .
$$

The solution to Eq. 120 in the across the $N+1$ interval may now be expressed.

$$
\begin{aligned}
q^{N}(x)= & q_{\text {general }}+q_{\text {particular }} \\
= & q_{N}+\left(\frac{d q}{d x}\right)_{N}\left(\eta(x)-\eta_{N}\right) \\
& +\frac{s_{N}}{\lambda_{N}}\left[\left(x-x_{N}\right)-\exp \left[-\frac{\lambda_{N}\left(x_{N}-x_{0}\right)}{\nu_{N}}\right]\left(\eta(x)-\eta_{N}\right)\right]
\end{aligned}
$$


The requirement for $\mathrm{C} 1$ continuity across each segment yields the following relations in which the initial value of $q$ and its gradient on segment $N$ is equal to the respective terminal values from the previous segment.

$$
\begin{aligned}
q_{N} & =q^{N-1}\left(x_{N}\right) \\
\left(\frac{d q}{d x}\right)_{N} & =\frac{d q^{N-1}\left(x_{N}\right)}{d x}
\end{aligned}
$$

The values for $\lambda_{N}$ and $\nu_{N}$ are algebraic functions of $q_{N}$ (and possibly $\left(\frac{d q}{d x}\right)_{N}$ ). The reconstruction algorithm may now be implemented as follows. First, integrate toward $x_{L}$. Initial values for $q_{0}$ and $\left(\frac{d q}{d x}\right)_{0}$ are specified and corresponding values for $\lambda_{0}, \nu_{0}$, and $s_{0}$ are computed. Segment 0 from $x_{0}$ to $x_{1}$ is defined such that $\operatorname{Re}\left(x_{1}-x_{0}\right)$ is of order 1 or smaller. Values for $q_{1}$ and $\left(\frac{d q}{d x}\right)_{1}$ are computed from Eq. 129 for $N=0$. Again, updated values for $\lambda_{1}, \nu_{1}$, and $s_{1}$ are computed. The integration continues in this manner segment by segment until $x_{L}$ is reached or until $\left|\eta_{N}\right|$ exceeds a specified ceiling. Record the value of $q_{L}^{*}$ at this terminal location. Repeat the integration using the same initial values to $x_{R}$ and record the value of $q_{R}^{*}$ for the terminal location to the right. Perturbations to initial values are introduced in a shooting algorithm until $\left(q_{L}^{*}-q_{L}\right)^{2}+\left(q_{R}^{*}-q_{R}\right)^{2}<\epsilon$. The initial values for $q_{0}^{*}$ and $\left(\frac{d q}{d x}\right)_{0}^{*}$ that satisfy the inequality are used to construct the interface flux. This algorithm enables a more accurate resolution of a steep transition between adjacent mesh points as compared to the constant wave speed approximation of Sec. III-A or the endpoint wave speed approximation of Sec. III-B. Shooting algorithms generally carry significant overhead in terms of convergence time and algorithm robustness.

\section{B. Linear Wave Speed Approximation}

The linear wave speed approximation is an interface centered formulation as is the constant wave speed approximation of Sec. III-A but it includes the gradient of wave speed to better approximate high gradient transitions through $\lambda_{0}=0$. Model Equation 5 is approximated over the interval $\left[x_{L}, x_{R}\right]$ by

$$
\left(\lambda_{0}+\lambda_{0}^{\prime} \frac{x-x_{0}}{2}\right) \frac{d q}{d x}=\nu_{0} \frac{d^{2} q}{d x^{2}}+s_{0}
$$

where $\lambda_{x, 0}=\left(\frac{d \lambda}{d x}\right)_{0}$. The source term $s$ and diffusivity $\nu$ are treated as constants across the interval $\left[x_{L}, x_{R}\right]$. The wave speed $\lambda$ is expanded as a linear function in $x$ to better characterize the reconstruction of diffusion terms in the limit of large cell Reynolds numbers, $R e_{x-x_{0}}=\lambda\left(x-x_{0}\right) / \nu$ as will be highlighted subsequently. Linear (or higher) expansions of the source term and diffusivity may introduce better reconstruction at the expense of a more complex relaxation algorithm. Such possibilities have not yet been explored. A semi-analytic solution to Eq. 132 (some numerical quadrature is required in the case of non-zero source terms) is derived by introducing the following map from $x$ to $\eta$.

$$
\begin{array}{ccc}
\eta & =\int_{0}^{x-x_{0}} \exp \left[\left(\lambda_{0}+\lambda_{x, 0} \frac{x^{\prime}}{2}\right) \frac{x^{\prime}}{\nu_{0}}\right] d x^{\prime} \\
\frac{d \eta}{d x} & = & \exp \left[\left(\lambda_{0}+\lambda_{x, 0} \frac{x-x_{0}}{2}\right) \frac{x-x_{0}}{\nu_{0}}\right] \\
\frac{d^{2} \eta}{d x^{2}} & = & \frac{\lambda_{0}+\lambda_{x, 0}\left(x-x_{0}\right)}{\nu_{0}} \frac{d \eta}{d x}
\end{array}
$$

Substitution of Eq. 133 into Eq. 132 yields

$$
\frac{d^{2} q}{d \eta^{2}}=-\frac{s_{0}}{\nu_{0}} \exp \left[-2\left(\lambda_{0}+\lambda_{x, 0} \frac{x-x_{0}}{2}\right) \frac{x-x_{0}}{\nu_{0}}\right]
$$


The general solution to Eq. 134 is

$$
\begin{aligned}
q_{\text {general }} & =q_{0}+\left(\frac{d q}{d \eta}\right)_{0} \eta \\
& =q_{0}+\left(\frac{d q}{d x}\right)_{0}\left(\frac{d x}{d \eta}\right)_{0} \eta \\
& =q_{0}+\left(\frac{d q}{d x}\right)_{0} \int_{0}^{x-x_{0}} \exp \left[\left(\lambda_{0}+\lambda_{x, 0} \frac{x^{\prime}}{2}\right) \frac{x^{\prime}}{\nu_{0}}\right] d x^{\prime}
\end{aligned}
$$

This formulation of a semi-analytic reconstruction requires quadrature to evaluate the transformed variable $\eta$. The formulation of the particular solution is not developed in detail here but one can follow the example of previous sections in order to see that two such quadratures would be required. The overhead here is quite similar to that encountered with the shooting algorithm of the previous section. For the case with $s_{0}=0$ the reconstruction is implemented as detailed in Eqs. $15-20$ using the present formulation of $\eta$. However, the Newton iteration in Eq. 19 must be set up as a function of two variables because $\eta$ is a function of both $\lambda_{0}\left(q_{0}\right)$ and $\lambda_{x, 0}\left(\left(\frac{d q}{d x}\right)_{0}\right)$.

\section{Appendix B - Vectors and Matrices for Multidimensional, Navier-Stokes Equations}

\section{A. Perfect Gas}

The vector of conserved variables $\mathbf{q}$ and primitive variables $\tilde{\mathbf{q}}$ are defined:

$$
\begin{aligned}
\mathbf{q}= & {\left[\begin{array}{c}
\rho \\
\rho U \\
\rho V \\
\rho W \\
\rho E
\end{array}\right] } \\
\tilde{\mathbf{q}}= & {\left[\begin{array}{c}
\rho \\
U \\
V \\
W \\
T
\end{array}\right] }
\end{aligned}
$$

Transformations from primitive to conservative variables $(\mathbf{T} d \tilde{\mathbf{q}}=d \mathbf{q})$ and conservative to primitive $\left(\mathbf{T}^{-1} d \mathbf{q}=d \tilde{\mathbf{q}}\right)$ are defined

$$
\begin{aligned}
\mathbf{T}= & {\left[\begin{array}{ccccc}
1 & 0 & 0 & 0 & 0 \\
U & \rho & 0 & 0 & 0 \\
V & 0 & \rho & 0 & 0 \\
W & 0 & 0 & \rho & 0 \\
E & \rho U & \rho V & \rho W & \rho C_{v}
\end{array}\right] } \\
\mathbf{T}^{-1}= & {\left[\begin{array}{ccccc}
1 & 0 & 0 & 0 & 0 \\
-\frac{U}{\rho} & \frac{1}{\rho} & 0 & 0 & 0 \\
-\frac{V}{\rho} & 0 & \frac{1}{\rho} & 0 & 0 \\
-\frac{W}{\rho} & 0 & 0 & \frac{1}{\rho} & 0 \\
\frac{1}{\rho C_{v}}(\tilde{\alpha}-e) & -\frac{U}{\rho C_{v}} & -\frac{V}{\rho C_{v}} & -\frac{W}{\rho C_{v}} & \frac{1}{\rho C_{v}}
\end{array}\right] }
\end{aligned}
$$


where $\tilde{\alpha}=\frac{1}{2}\left(U^{2}+V^{2}+W^{2}\right), E=e+\tilde{\alpha}$, and $d e=C_{v} d T$.

The linearization employs matrices $\mathbf{A}$ and $\tilde{\mathbf{B}}$ defined below.

$$
\begin{gathered}
\frac{d \mathbf{f}_{1}}{d \mathbf{q}}=\mathbf{A}_{1}=\left[\begin{array}{ccccc}
0 & 1 & 0 & 0 & 0 \\
\tilde{\beta} \tilde{\alpha}-U^{2} & -\tilde{\beta} U+2 U & -\tilde{\beta} V & -\tilde{\beta} W & \tilde{\beta} \\
-U V & V & U & 0 & 0 \\
-U W & W & 0 & U & 0 \\
\tilde{\beta} \tilde{\alpha} U-U H & -\tilde{\beta} U^{2}+H & -\tilde{\beta} U V & -\tilde{\beta} U W & \tilde{\beta} U+U
\end{array}\right] \\
\frac{d \mathbf{h}_{1}}{d\left(\frac{d \tilde{\mathbf{q}}}{d X}\right)}=\nu \tilde{\mathbf{B}}_{11}=\nu \\
{\left[\begin{array}{ccccc}
0 & 0 & 0 & 0 & 0 \\
0 & \frac{4}{3} \rho & 0 & 0 & 0 \\
0 & 0 & \rho & 0 & 0 \\
0 & 0 & 0 & \rho & 0 \\
0 & \frac{4}{3} \rho U & \rho V & \rho W & \frac{\rho C_{p}}{\operatorname{Pr}}
\end{array}\right]}
\end{gathered}
$$

Diagonalization of $\mathbf{A}_{1}=\mathbf{R}_{1} \boldsymbol{\Lambda}_{1} \mathbf{R}_{1}^{-1}$ is implemented with

$$
\begin{aligned}
& \mathbf{R}_{1}= \frac{1}{2 c^{2}}\left[\begin{array}{ccccc}
2 & 0 & 0 & 1 & 1 \\
2 U & 0 & 0 & U+c & U-c \\
2 V & 2 c^{2} & 0 & V & V \\
2 W & 0 & 2 c^{2} & W & W \\
2 \tilde{\alpha} & 2 V c^{2} & 2 W c^{2} & H+c U & H-c U
\end{array}\right] \\
& \mathbf{R}_{1}^{-1}= {\left[\begin{array}{cccccc}
c^{2}-\tilde{\beta} \tilde{\alpha} & \tilde{\beta} U & \tilde{\beta} V & \tilde{\beta} W & -\tilde{\beta} \\
-V & 0 & 1 & 0 & 0 \\
-W & & 0 & 0 & 1 & 0 \\
\tilde{\beta} \tilde{\alpha}-U c & c-\tilde{\beta} U & -\tilde{\beta} V & -\tilde{\beta} W & \tilde{\beta} \\
\tilde{\beta} \tilde{\alpha}+U c & -c-\tilde{\beta} U & -\tilde{\beta} V & -\tilde{\beta} W & \tilde{\beta}
\end{array}\right] } \\
& \Lambda_{1}=\left[\begin{array}{cccccc}
U & 0 & 0 & 0 & 0 & \\
0 & U & 0 & 0 & 0 & \\
0 & 0 & U & 0 & 0 & 0 \\
0 & 0 & 0 & U+c & 0 \\
0 & 0 & 0 & 0 & U-c
\end{array}\right]
\end{aligned}
$$

The corresponding matrices in the $Y$ direction are:

$$
\begin{gathered}
\frac{d \mathbf{f}_{2}}{d \mathbf{q}}=\mathbf{A}_{2}=\left[\begin{array}{ccccc}
0 & 0 & 1 & 0 & 0 \\
-V U & V & U & 0 & 0 \\
\tilde{\beta} \tilde{\alpha}-V^{2} & -\tilde{\beta} U & -\tilde{\beta} V+2 V & -\tilde{\beta} W & \tilde{\beta} \\
-V W & 0 & W & V & 0 \\
\tilde{\beta} \tilde{\alpha} V-V H & -\tilde{\beta} V U & -\tilde{\beta} V^{2}+H & -\tilde{\beta} V W & \tilde{\beta} V+V
\end{array}\right] \\
\frac{d \mathbf{h}_{2}}{d\left(\frac{d \tilde{\mathbf{q}}}{d Y}\right)}=\nu \tilde{\mathbf{B}}_{22}= \\
{\left[\begin{array}{ccccc}
0 & 0 & 0 & 0 & 0 \\
0 & \rho & 0 & 0 & 0 \\
0 & 0 & \frac{4}{3} \rho & 0 & 0 \\
0 & 0 & 0 & \rho & 0 \\
0 & \rho U & \frac{4}{3} \rho V & \rho W & \frac{\rho C_{p}}{\operatorname{Pr}}
\end{array}\right]} \\
47 \text { of } 52
\end{gathered}
$$


Diagonalization of $\mathbf{A}_{2}=\mathbf{R}_{2} \boldsymbol{\Lambda}_{2} \mathbf{R}_{2}^{-1}$ is implemented with

$$
\begin{aligned}
& \mathbf{R}_{2}=\frac{1}{2 c^{2}}\left[\begin{array}{ccccc}
2 & 0 & 0 & 1 & 1 \\
2 U & 0 & 2 c^{2} & U & U \\
2 V & 0 & 0 & V+c & V-c \\
2 W & 2 c^{2} & 0 & W & W \\
2 \tilde{\alpha} & 2 W c^{2} & 2 U c^{2} & H+c V & H-c V
\end{array}\right] \\
& \mathbf{R}_{2}^{-1}= {\left[\begin{array}{cccccc}
c^{2}-\tilde{\beta} \tilde{\alpha} & \tilde{\beta} U & \tilde{\beta} V & \tilde{\beta} W & -\tilde{\beta} \\
-W & 0 & 0 & 1 & 0 \\
-U & 1 & 0 & 0 & 0 \\
\tilde{\beta} \tilde{\alpha}-V c & -\tilde{\beta} U & c-\tilde{\beta} V & -\tilde{\beta} W & \tilde{\beta} \\
\tilde{\beta} \tilde{\alpha}+V c & -\tilde{\beta} U & -c-\tilde{\beta} V & -\tilde{\beta} W & \tilde{\beta}
\end{array}\right] } \\
& \Lambda_{2}=\left[\begin{array}{cccccc}
V & 0 & 0 & 0 & 0 & \\
0 & V & 0 & 0 & 0 & \\
0 & 0 & V & 0 & 0 & \\
0 & 0 & 0 & V+c & 0 \\
0 & 0 & 0 & 0 & V-c
\end{array}\right]
\end{aligned}
$$

The corresponding matrices in the $Z$ direction are:

$$
\begin{gathered}
\frac{d \mathbf{f}_{3}}{d \mathbf{q}}=\mathbf{A}_{3}=\left[\begin{array}{ccccc}
0 & 0 & 0 & c_{s} & 0 \\
-W U & W & 0 & U & 0 \\
-W V & 0 & W & V & 0 \\
\tilde{\beta} \tilde{\alpha}-W^{2} & -\tilde{\beta} U & -\tilde{\beta} V & -\tilde{\beta} W+2 W & \tilde{\beta} \\
\tilde{\beta} \tilde{\alpha} W-W H & -\tilde{\beta} W U & -\tilde{\beta} W V & -\tilde{\beta} W^{2}+H & \tilde{\beta} W+W
\end{array}\right] \\
\frac{d \mathbf{h}_{3}}{d\left(\frac{d \tilde{\mathbf{q}}}{d Z}\right)}=\nu \tilde{\mathbf{B}}_{33}=\left[\begin{array}{ccccc}
0 & 0 & 0 & 0 & 0 \\
0 & \rho & 0 & 0 & 0 \\
0 & 0 & \rho & 0 & 0 \\
0 & 0 & 0 & \frac{4}{3} \rho & 0 \\
0 & \rho U & \rho V & \frac{4}{3} \rho W & \frac{\rho C_{p}}{\operatorname{Pr}}
\end{array}\right]
\end{gathered}
$$

Diagonalization of $\mathbf{A}_{3}=\mathbf{R}_{3} \boldsymbol{\Lambda}_{3} \mathbf{R}_{3}^{-1}$ is implemented with

$$
\begin{aligned}
\mathbf{R}_{3}=\frac{1}{2 c^{2}} & {\left[\begin{array}{ccccc}
2 & 0 & 0 & 1 & 1 \\
2 U & 2 c^{2} & 0 & U & U \\
2 V & 0 & 2 c^{2} & V & V \\
2 W & 0 & 0 & W+c & W-c \\
2 \tilde{\alpha} & 2 W c^{2} & 2 U c^{2} & H+c W & H-c W
\end{array}\right] } \\
\mathbf{R}_{3}^{-1}= & {\left[\begin{array}{ccccc}
c^{2}-\tilde{\beta} \tilde{\alpha} & \tilde{\beta} U & \tilde{\beta} V & \tilde{\beta} W & -\tilde{\beta} \\
-U & 1 & 0 & 0 & 0 \\
-V & 0 & 1 & 0 & 0 \\
\tilde{\beta} \tilde{\alpha}-W c & -\tilde{\beta} U & -\tilde{\beta} V & c-\tilde{\beta} W & \tilde{\beta} \\
\tilde{\beta} \tilde{\alpha}+W c & -\tilde{\beta} U & -\tilde{\beta} V & -c-\tilde{\beta} W & \tilde{\beta}
\end{array}\right] }
\end{aligned}
$$




$$
\mathbf{\Lambda}_{3}=\left[\begin{array}{ccccc}
W & 0 & 0 & 0 & 0 \\
0 & W & 0 & 0 & 0 \\
0 & 0 & W & 0 & 0 \\
0 & 0 & 0 & W+c & 0 \\
0 & 0 & 0 & 0 & W-c
\end{array}\right]
$$

For convenience, the product $\mathbf{R}^{-1} \tilde{\mathbf{B}} \mathbf{T}^{-1} \mathbf{R}$ is defined below.

$$
\mathbf{R}^{-1} \tilde{\mathbf{B}} \mathbf{T}^{-1} \mathbf{R}=\frac{1}{2 \operatorname{Pr}}\left[\begin{array}{ccccc}
2 & 0 & 0 & -\tilde{\beta} & -\tilde{\beta} \\
0 & 2 \operatorname{Pr} & 0 & 0 & 0 \\
0 & 0 & 2 \operatorname{Pr} & 0 & 0 \\
-2 & 0 & 0 & \tilde{\beta}+\frac{4}{3} \operatorname{Pr} & \tilde{\beta}-\frac{4}{3} \operatorname{Pr} \\
-2 & 0 & 0 & \tilde{\beta}-\frac{4}{3} \operatorname{Pr} & \tilde{\beta}+\frac{4}{3} \operatorname{Pr}
\end{array}\right]
$$

\section{B. Mixture of Thermally Perfect Gases}

The vector of conserved variables $\mathbf{q}$ and primitive variables $\tilde{\mathbf{q}}$ are defined:

$$
\begin{aligned}
\mathbf{q}= & {\left[\begin{array}{c}
\rho_{s} \\
\rho U \\
\rho V \\
\rho W \\
\rho E \\
\rho e_{v}
\end{array}\right] } \\
\tilde{\mathbf{q}}= & {\left[\begin{array}{c}
\rho_{s} \\
U \\
V \\
W \\
T \\
T_{v}
\end{array}\right] }
\end{aligned}
$$

Transformations from primitive to conservative variables $(\mathbf{T} d \tilde{\mathbf{q}}=d \mathbf{q})$ and conservative to primitive $\left(\mathbf{T}^{-1} d \mathbf{q}=d \tilde{\mathbf{q}}\right)$ are defined

$$
\begin{aligned}
& \mathbf{T}=\left[\begin{array}{cccccc}
\delta_{s, r} & 0 & 0 & 0 & 0 & 0 \\
U & \rho & 0 & 0 & 0 & 0 \\
V & 0 & \rho & 0 & 0 & 0 \\
W & 0 & 0 & \rho & 0 & 0 \\
E & \rho U & \rho V & \rho W & \rho C_{v, t r} & \rho C_{v, v} \\
e_{v} & 0 & 0 & 0 & 0 & \rho C_{v, v}
\end{array}\right] \\
& \mathbf{T}^{-1}=\left[\begin{array}{cccccc}
\delta_{s, r} & 0 & 0 & 0 & 0 & 0 \\
-\frac{U}{\rho} & \frac{1}{\rho} & 0 & 0 & 0 & 0 \\
-\frac{V}{\rho} & 0 & \frac{1}{\rho} & 0 & 0 & 0 \\
-\frac{W}{\rho} & 0 & 0 & \frac{1}{\rho} & 0 & 0 \\
\frac{1}{\rho C_{v, t r}}\left(\tilde{\alpha}-e_{t r}\right) & -\frac{U}{\rho C_{v, t r}} & -\frac{V}{\rho C_{v, t r}} & -\frac{W}{\rho C_{v, t r}} & \frac{1}{\rho C_{v, t r}} & -\frac{1}{\rho C_{v, t r}} \\
-\frac{e_{v}}{\rho C_{v, v}} & 0 & 0 & 0 & 0 & \frac{1}{\rho C_{v, v}}
\end{array}\right]
\end{aligned}
$$

49 of 52 
The linearization employs matrices $\mathbf{A}$ and $\tilde{\mathbf{B}}$ defined below.

$$
\begin{gathered}
\frac{d \mathbf{f}_{1}}{d \mathbf{q}}=\mathbf{A}_{1}=\left[\begin{array}{cccccc}
U\left(\delta_{s, r}-c_{s}\right) & c_{s} & 0 & 0 & 0 & 0 \\
\tilde{\gamma}_{r}-U^{2} & -\tilde{\beta} U+2 U & -\tilde{\beta} V & -\tilde{\beta} W & \tilde{\beta} & \tilde{\phi} \\
-U V & V & U & 0 & 0 & 0 \\
-U W & W & 0 & U & 0 & 0 \\
\tilde{\gamma}_{r} U-U H & -\tilde{\beta} U^{2}+H & -\tilde{\beta} U V & -\tilde{\beta} U W & \tilde{\beta} U+U & \tilde{\phi} U \\
-U e_{V} & e_{V} & 0 & 0 & 0 & U
\end{array}\right] \\
\frac{d \mathbf{h}_{1}}{d\left(\frac{d \tilde{\mathbf{q}}}{d X}\right)}=\nu \tilde{\mathbf{B}}_{11}=\nu\left[\begin{array}{cccccc}
\frac{\xi_{s}}{\mathrm{Sc}_{s}}\left(\delta_{s, r}-c_{s} \xi_{r}\right) & 0 & 0 & 0 & 0 & 0 \\
0 & \frac{4}{3} \rho & 0 & 0 & 0 & 0 \\
0 & 0 & \rho & 0 & 0 & 0 \\
0 & 0 & 0 & \rho & 0 & 0 \\
\xi_{r}\left(\frac{h_{t r, r}}{\mathrm{Sc}_{r}}-\left\langle\frac{h_{t r}}{\mathrm{Sc}}\right\rangle\right) & \frac{4}{3} \rho U & \rho V & \rho W & \frac{\rho C_{p}}{\operatorname{Pr}_{t r}} & \frac{\rho C_{p}}{\operatorname{Pr}_{v}} \\
\xi_{r}\left(\frac{h_{v, r}}{\mathrm{Sc}_{r}}-\left\langle\frac{h_{v}}{\mathrm{Sc}}\right\rangle\right) & 0 & 0 & 0 & 0 & \frac{\rho C_{p}}{\operatorname{Pr}_{v}}
\end{array}\right]
\end{gathered}
$$

where $\xi_{s}=\tilde{M} / \tilde{M}_{s}$ if diffusion of mass is driven by gradients of mole fraction or $\xi_{s}=1$ if diffusion of mass is driven by gradients of mass fraction. The Schmidt number for species $s$ is defined $\mathrm{Sc}_{s}=\mu / \rho D_{s}$. The Prandtl number for conduction of translational-rotational energy is defined $\operatorname{Pr}_{t r}=\mu C_{p} / \tilde{\eta}_{t r}$ and Prandtl number for conduction of vibrational-electronic energy is defined $\operatorname{Pr}_{v}=\mu C_{p} / \tilde{\eta}_{v}$. The evaluation of the Jacobian for diffusion of energy requires a summation over the product of mass diffusion flux times species enthalpies which is represented by $\left\langle\frac{h_{t r}}{\mathrm{Sc}}\right\rangle=$ $\sum_{s} c_{s} \xi_{s} h_{t r, s} / \mathrm{Sc}_{s}$ and by $\left\langle\frac{h_{v}}{\mathrm{Sc}}\right\rangle=\sum_{s} c_{s} \xi_{s} h_{v, s} / \mathrm{Sc}_{s}$.

Diagonalization of $\mathbf{A}_{1}=\mathbf{R}_{1} \boldsymbol{\Lambda}_{1} \mathbf{R}_{1}^{-1}$ is implemented with

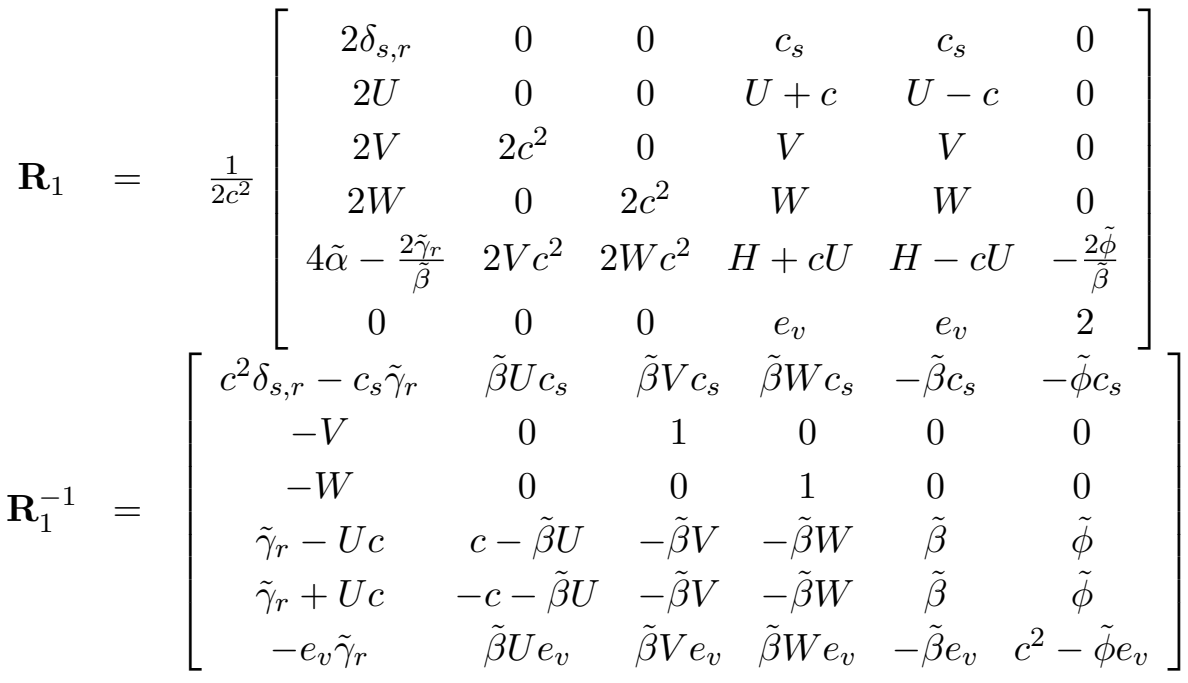

$$
\begin{aligned}
& \boldsymbol{\Lambda}_{1}=\left[\begin{array}{cccccc}
U & 0 & 0 & 0 & 0 & 0 \\
0 & U & 0 & 0 & 0 & 0 \\
0 & 0 & U & 0 & 0 & 0 \\
0 & 0 & 0 & U+c & 0 & 0 \\
0 & 0 & 0 & 0 & U-c & 0 \\
0 & 0 & 0 & 0 & 0 & U
\end{array}\right]
\end{aligned}
$$


The corresponding matrices in the $Y$ direction are:

$$
\begin{gathered}
\frac{d \mathbf{f}_{2}}{d \mathbf{q}}=\mathbf{A}_{2}=\left[\begin{array}{cccccc}
V\left(\delta_{s, r}-c_{s}\right) & 0 & c_{s} & 0 & 0 & 0 \\
-V U & V & U & 0 & 0 & 0 \\
\tilde{\gamma}_{r}-V^{2} & -\tilde{\beta} U & -\tilde{\beta} V+2 V & -\tilde{\beta} W & \tilde{\beta} & \tilde{\phi} \\
-V W & 0 & W & V & 0 & 0 \\
\tilde{\gamma}_{r} V-V H & -\tilde{\beta} V U & -\tilde{\beta} V^{2}+H & -\tilde{\beta} V W & \tilde{\beta} V+V & \tilde{\phi} V \\
-V e_{v} & 0 & e_{v} & 0 & 0 & V
\end{array}\right] \\
\frac{d \mathbf{h}_{2}}{d\left(\frac{d \tilde{\mathbf{q}}}{d Y}\right)}=\nu \tilde{\mathbf{B}}_{22}=\left[\begin{array}{cccccc}
\frac{\xi_{s}}{\mathrm{Sc}_{s}}\left(\delta_{s, r}-c_{s} \xi_{r}\right) & 0 & 0 & 0 & 0 & 0 \\
0 & \rho & 0 & 0 & 0 & 0 \\
0 & 0 & \frac{4}{3} \rho & 0 & 0 & 0 \\
0 & 0 & 0 & \rho & 0 & 0 \\
\xi_{r}\left(\frac{h_{t r, r}}{\mathrm{Sc}}-\left\langle\frac{h_{t r}}{\mathrm{Sc}}\right\rangle\right) & \rho U & \frac{4}{3} \rho V & \rho W & \frac{\rho C_{p}}{\operatorname{Pr}_{t r}} & \frac{\rho C_{p}}{\operatorname{Pr}_{v}} \\
\xi_{r}\left(\frac{h_{v, r}}{\mathrm{Sc}_{r}}-\left\langle\frac{h_{v}}{\mathrm{Sc}}\right\rangle\right) & 0 & 0 & 0 & 0 & \frac{\rho C_{p}}{\operatorname{Pr}_{v}}
\end{array}\right]
\end{gathered}
$$

Diagonalization of $\mathbf{A}_{2}=\mathbf{R}_{2} \boldsymbol{\Lambda}_{2} \mathbf{R}_{2}^{-1}$ is implemented with

$$
\begin{aligned}
& \mathbf{R}_{2}=\frac{1}{2 c^{2}}\left[\begin{array}{cccccc}
2 \delta_{s, r} & 0 & 0 & c_{s} & c_{s} & 0 \\
2 U & 0 & 2 c^{2} & U & U & 0 \\
2 V & 0 & 0 & V+c & V-c & 0 \\
2 W & 2 c^{2} & 0 & W & W & 0 \\
4 \tilde{\alpha}-\frac{2 \tilde{\gamma}_{r}}{\tilde{\beta}} & 2 W c^{2} & 2 U c^{2} & H+c V & H-c V & -\frac{2 \tilde{\phi}}{\tilde{\beta}} \\
0 & 0 & 0 & e_{v} & e_{v} & 2
\end{array}\right] \\
& \mathbf{R}_{2}^{-1}=\left[\begin{array}{cccccc}
c^{2} \delta_{s, r}-c_{s} \tilde{\gamma}_{r} & \tilde{\beta} U c_{s} & \tilde{\beta} V c_{s} & \tilde{\beta} W c_{s} & -\tilde{\beta} c_{s} & -\tilde{\phi} c_{s} \\
-W & 0 & 0 & 1 & 0 & 0 \\
-U & 1 & 0 & 0 & 0 & 0 \\
\tilde{\gamma}_{r}-V c & -\tilde{\beta} U & c-\tilde{\beta} V & -\tilde{\beta} W & \tilde{\beta} & \tilde{\phi} \\
\tilde{\gamma}_{r}+V c & -\tilde{\beta} U & -c-\tilde{\beta} V & -\tilde{\beta} W & \tilde{\beta} & \tilde{\phi} \\
-e_{v} \tilde{\gamma}_{r} & \tilde{\beta} U e_{v} & \tilde{\beta} V e_{v} & \tilde{\beta} W e_{v} & -\tilde{\beta} e_{v} & c^{2}-\tilde{\phi} e_{v}
\end{array}\right] \\
& \boldsymbol{\Lambda}_{2}=\left[\begin{array}{cccccc}
V & 0 & 0 & 0 & 0 & 0 \\
0 & V & 0 & 0 & 0 & 0 \\
0 & 0 & V & 0 & 0 & 0 \\
0 & 0 & 0 & V+c & 0 & 0 \\
0 & 0 & 0 & 0 & V-c & 0 \\
0 & 0 & 0 & 0 & 0 & V
\end{array}\right]
\end{aligned}
$$

The corresponding matrices in the $Z$ direction are:

$$
\frac{d \mathbf{f}_{3}}{d \mathbf{q}}=\mathbf{A}_{3}=\left[\begin{array}{cccccc}
W\left(\delta_{s, r}-c_{s}\right) & 0 & 0 & c_{s} & 0 & 0 \\
-W U & W & 0 & U & 0 & 0 \\
-W V & 0 & W & V & 0 & 0 \\
\tilde{\gamma}_{r}-W^{2} & -\tilde{\beta} U & -\tilde{\beta} V & -\tilde{\beta} W+2 W & \tilde{\beta} & \tilde{\phi} \\
\tilde{\gamma}_{r} W-W H & -\tilde{\beta} W U & -\tilde{\beta} W V & -\tilde{\beta} W^{2}+H & \tilde{\beta} W+W & \tilde{\phi} W \\
-W e_{v} & 0 & 0 & e_{v} & 0 & W
\end{array}\right]
$$




$$
\frac{d \mathbf{h}_{3}}{d\left(\frac{d \tilde{\mathbf{q}}}{d Z}\right)}=\nu \tilde{\mathbf{B}}_{33}=\left[\begin{array}{cccccc}
\frac{\xi_{s}}{\mathrm{Sc}_{s}}\left(\delta_{s, r}-c_{s} \xi_{r}\right) & 0 & 0 & 0 & 0 & 0 \\
0 & \rho & 0 & 0 & 0 & 0 \\
0 & 0 & \rho & 0 & 0 & 0 \\
0 & 0 & 0 & \frac{4}{3} \rho & 0 & 0 \\
\xi_{r}\left(\frac{h_{t r, r}}{\mathrm{Sc}_{r}}-\left\langle\frac{h_{t r}}{\mathrm{Sc}}\right\rangle\right) & \rho U & \rho V & \frac{4}{3} \rho W & \frac{\rho C_{p}}{\operatorname{Pr}_{t r}} & \frac{\rho C_{p}}{\operatorname{Pr}_{v}} \\
\xi_{r}\left(\frac{h_{v, r}}{\mathrm{Sc}_{r}}-\left\langle\frac{h_{v}}{\mathrm{Sc}}\right\rangle\right) & 0 & 0 & 0 & 0 & \frac{\rho C_{p}}{\operatorname{Pr}_{v}}
\end{array}\right]
$$

Diagonalization of $\mathbf{A}_{3}=\mathbf{R}_{3} \boldsymbol{\Lambda}_{3} \mathbf{R}_{3}^{-1}$ is implemented with

$$
\begin{aligned}
& \mathbf{R}_{3}=\frac{1}{2 c^{2}}\left[\begin{array}{cccccc}
2 \delta_{s, r} & 0 & 0 & c_{s} & c_{s} & 0 \\
2 U & 2 c^{2} & 0 & U & U & 0 \\
2 V & 0 & 2 c^{2} & V & V & 0 \\
2 W & 0 & 0 & W+c & W-c & 0 \\
4 \tilde{\alpha}-\frac{2 \tilde{\gamma}_{r}}{\tilde{\beta}} & 2 W c^{2} & 2 U c^{2} & H+c W & H-c W & -\frac{2 \tilde{\phi}}{\tilde{\beta}} \\
0 & 0 & 0 & e_{v} & e_{v} & 2
\end{array}\right] \\
& \mathbf{R}_{3}^{-1}=\left[\begin{array}{cccccc}
c^{2} \delta_{s, r}-c_{s} \tilde{\gamma}_{r} & \tilde{\beta} U c_{s} & \tilde{\beta} V c_{s} & \tilde{\beta} W c_{s} & -\tilde{\beta} c_{s} & -\tilde{\phi} c_{s} \\
-U & 1 & 0 & 0 & 0 & 0 \\
-V & 0 & 1 & 0 & 0 & 0 \\
\tilde{\gamma}_{r}-W c & -\tilde{\beta} U & -\tilde{\beta} V & c-\tilde{\beta} W & \tilde{\beta} & \tilde{\phi} \\
\tilde{\gamma}_{r}+W c & -\tilde{\beta} U & -\tilde{\beta} V & -c-\tilde{\beta} W & \tilde{\beta} & \tilde{\phi} \\
-e_{v} \tilde{\gamma}_{r} & \tilde{\beta} U e_{v} & \tilde{\beta} V e_{v} & \tilde{\beta} W e_{v} & -\tilde{\beta} e_{v} & c^{2}-\tilde{\phi} e_{v}
\end{array}\right] \\
& \boldsymbol{\Lambda}_{3}=\left[\begin{array}{cccccc}
W & 0 & 0 & 0 & 0 & 0 \\
0 & W & 0 & 0 & 0 & 0 \\
0 & 0 & W & 0 & 0 & 0 \\
0 & 0 & 0 & W+c & 0 & 0 \\
0 & 0 & 0 & 0 & W-c & 0 \\
0 & 0 & 0 & 0 & 0 & W
\end{array}\right]
\end{aligned}
$$

